
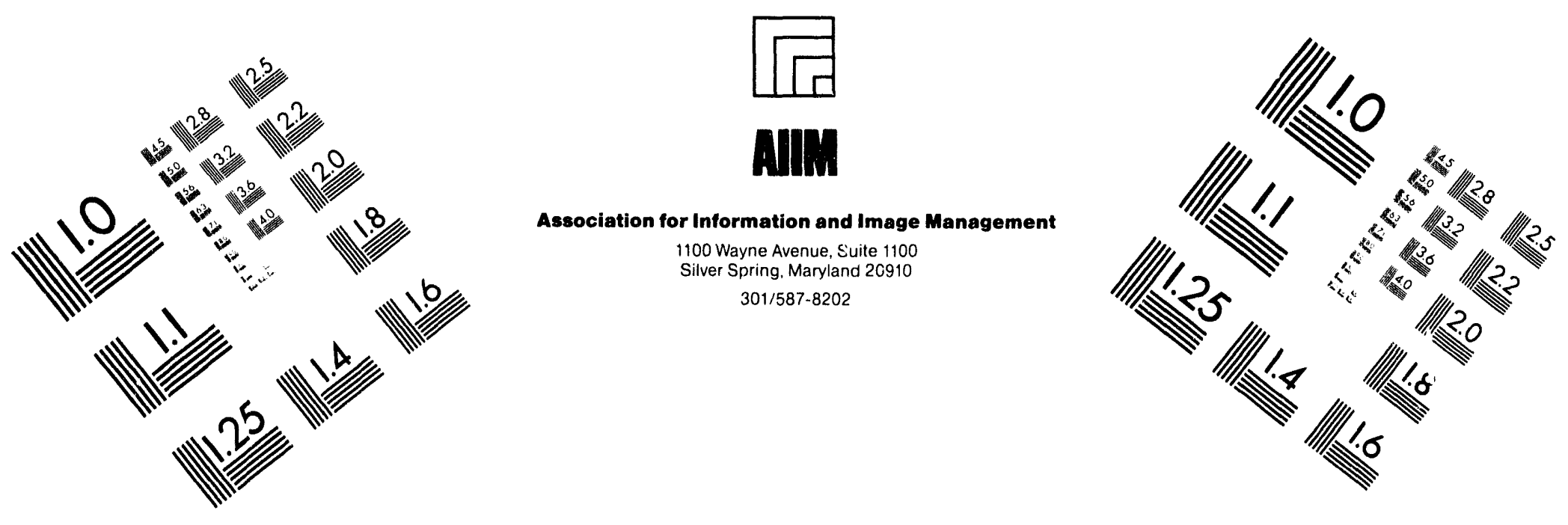

\title{
Centimeter
}

$\mid \begin{aligned} & 1 \\ & \mid\end{aligned}$ Inches

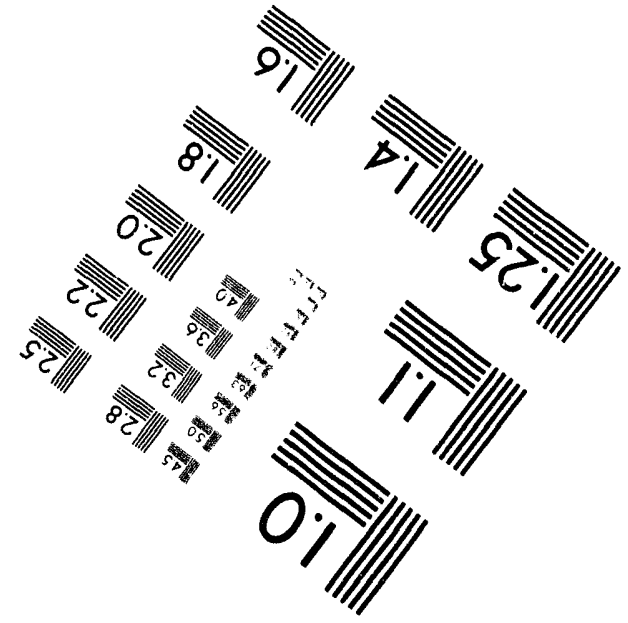

MANUFACTURED TO AIIM STANDARDS BY APPLIED IMAGE, INC.

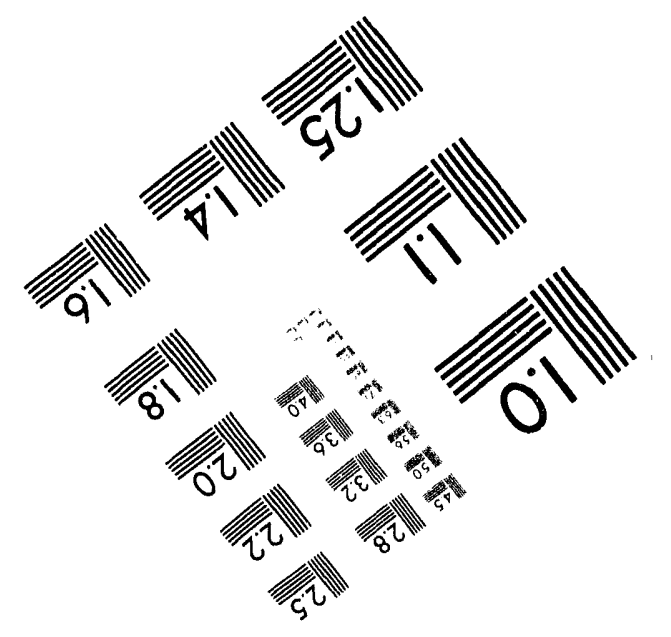



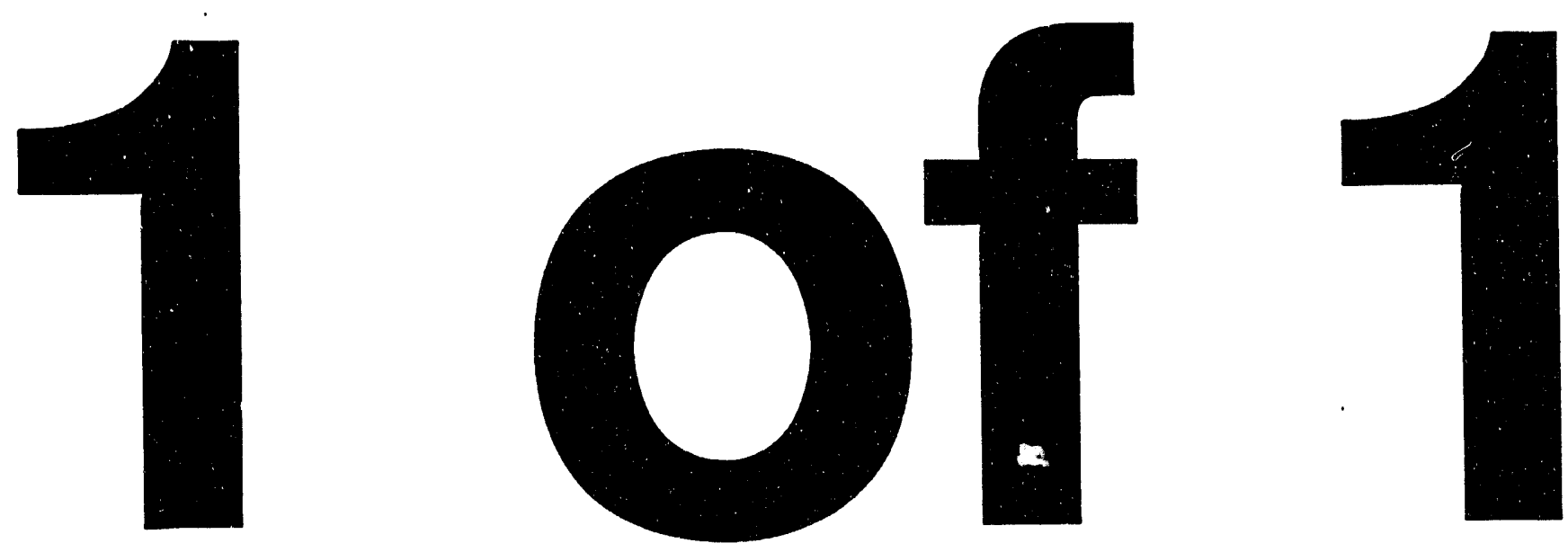


\section{FY 1993}

Report on

Lawrence

Berkeley

Laboratory

\section{Laboratory Directed Research and Development Program}

Lawrence Berkeley Laboratory

University of California

Berkeley California 94720

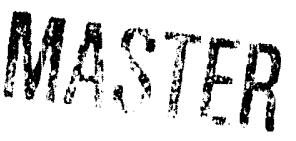




\section{Table of Contents}

Introduction

\section{Project Reports}

Accelerator and Fusion Research Division ........................................................................................... 1

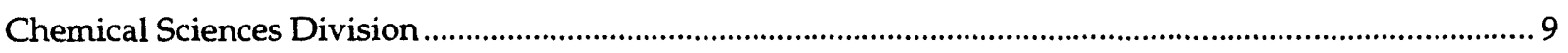

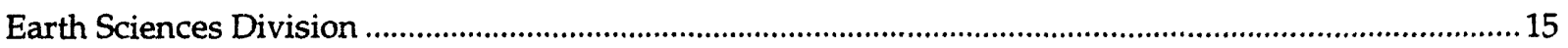

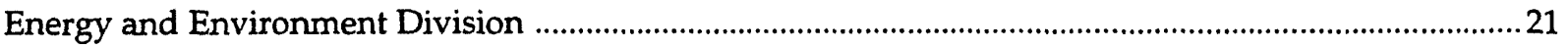

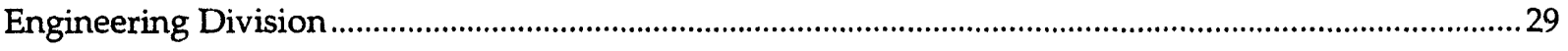

Environment, Health and Safety Division ........................................................................................... 33

Information and Computing Sciences Division ....................................................................................... 35

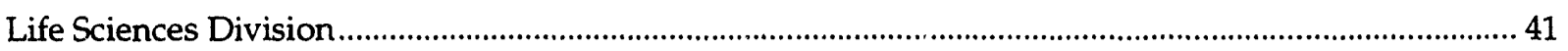

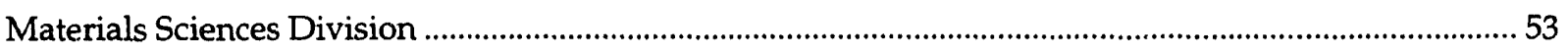

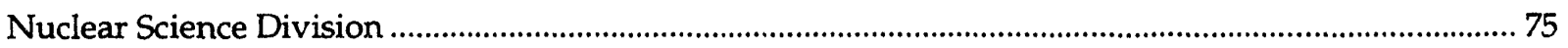

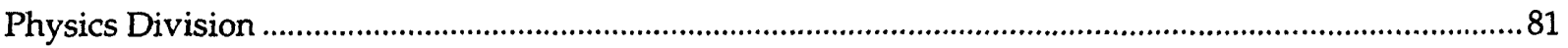

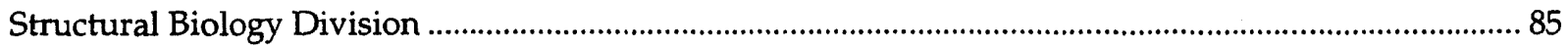

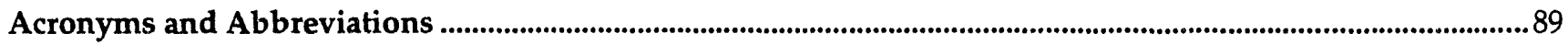




\section{Introduction}

The Lawrence Berkeley Laboratory (LBL) Laboratory Directed Research and Development Program FY 1993 report is compiled from annual reports submitted by principal investigators following the close of the fiscal year. This report describes the projects supported and summarizes their accomplishments. It constitutes a part of the Laboratory Directed Research and Development (LDRD) program planning and documentation process that includes an annual planning cycle, project selection, implementation, and review.

The LBL LDRD program is a critical tool for directing the Laboratory's forefront scientific research capabilities toward vital, excellent, and emerging scientific challenges. The program provides the resources for LBL scientists to make rapid and significant contributions to critical national science and technology problems. The LDRD also advances the Laboratory's core competencies, foundations, scientific capability, and permits exploration of exciting new opportunities. Areas eligible for support include:

- Work in forefront areas of science and technology that enrich Laboratory research and development capability;

- Advanced study of new hypotheses, new experiments, and innovative approaches to develop new concepts or knowledge;

- Experiments directed toward proof of principle for initial hypothesis testing or verification; and

- Conception and preliminary technical analysis to explore possible instrumentation, experimental facilities, or new devices.

The LDRD program supports LBL's mission in many ways. First, because LDRD funds can be allocated in a relatively short time frame, LBL researchers can support the mission of DOE and serve the needs of the nation by quickly responding to forefront scientific problems. Second, LDRD enables the Laboratory to attract and retain highly qualified scientists, and supports their efforts to carry out world-leading research. Finally, the LDRD program also supports new projects that involve graduate students and postdoctoral fellows, thus contributing to the education mission of the Laboratory.

LBL has a formal process for allocation of funds for LDRD. The process relies on individual scientific investigators and the scientific leadership of the Laboratory to identify opportunities that will contribute to scientific and institutional goals. The process is also designed to maintain compliance with DOE Orders, in particular DOE Order 5000.4A. From year to year, the distribution of funds among the scientific program areas will change. This flexibility optimizes the Laboratory's ability to respond to opportunities.

Laboratory LDRD policy and program decisions are the responsibility of the Laboratory Director. The Director has assigned general programmatic oversight responsibility to the Laboratory Deputy Director. Administration and reporting on the LDRD program is supported by the Office for Planning and Development. LDRD accounting procedures and financial management are consistent with the Laboratory's accounting principles and stipulations under the contract between the University of California and the Department of Energy, with accounting maintained through the Laboratory's Chief Financial Officer.

In FY 1993, LBL was authorized by the Department of Energy to establish a funding ceiling for LDRD of $3 \%$ of the I aboratory's FY 1993 operating budget. This funding level was provided to develop new scientific ideas and opportunities and allow the Laboratory Director an opportunity to initiate new directions. However, budget constraints limited available resources, so only $\$ 5.1 \mathrm{M}$ was allocated.

In FY 1993, scientists submitted 110 proposals requesting over $\$ 12 \mathrm{M}$. A total of 49 projects were funded in FY 1993, with awards rang ing from $\$ 15 \mathrm{~K}$ to $\$ 485 \mathrm{~K}$. These projects are summarized in Table 1. 
Table 1. FY 1993 Laboratory Directed Research and Development Program.

\section{Accelerator and Fusion Research Division}

J.W. Staples

B.A. Ludewigt

J.R. Alonso

D.R. Dietderich

R.A. Gough

M. Xie
Bearn Scanning: Modeling and System Specifications for an Integrated

3-D I'roton Treatment-Delivery System

49,800

Phase Equilibria and Microstructural Texture in Bi-Oxide High- $\mathrm{T}_{\mathrm{c}}$ Superconductors

50,800

Scattering Techniques for Femtosec' ind X-Ray Pulses

Study of Coherent Radiation Sources beyond the ALS

477,700

199,300

\section{Chemical Sciences Division}
R.G. Bergman
B.M. Novak
T.Y. Meyer
J. Yu
C.B. Harris
D. K. Shuh

Exploration of the Interface between Homogeneous and Heterogeneous Catalysis: Synthesis and Study of Organometallic Catalysts Supported on Novel Polysiloxane Materials with Controllable Solubility Properties

Ultrafast Dynamics of Electrons at Surfaces 100,400

Structure and Chemistry of Nonmetallic Adsorbates at Compound Semiconductor Interfaces Investigated by Synchrotron Radiation Techniques

\section{Earth Sciences Division}
J.T. Geller
Exploratory Research on Seismic Imaging of Organic Liquid
L.R. Myer
Contaminants in Unconsolidated Media
T.K. Tokunaga
X-Ray Absorption Spectroscopy and X-Ray Microprobe Studies of Contaminated Soils

\section{Energy and Environment Division}

S.P. Cramer

J. van Elp

J.L. Moore

C.R. Randal

$X$. Wang

D.F. Grether

H. Akbari

A.H. Rosenfeld

P.P. Varaiya

F.F. Wu

C. Blumstein
Exploratory R\&D Flow-Pump Probe X-Ray Absorption and Emission 
Table 1. Continued.

Division Investigator Project Title

(\$)

A.K. Oppenheim Inverse Problem to Direct Modeling of Combustion in Engines

R.L. Ritschard

New Approaches to Assessing Oceans as Carbon Dioxide Sinks

104,300

M. Quinby-Hunt

K.H. Orvis

P. Neale

M.D. Rubin

Materials Processing and Device Fabrication of Wide-Bandgap Materials

90,000

Engineering Division

J.M. Jaklevic

J.C. Schultz

Development of Microchemical Methods for Biological Assays

46,900

S.A. Kleinfelder

Custom Gallium Arsenide Integrated Circuit Design for Applications

E. Beuville in Nuclear and Particle Physics

70,000

O.B. Milgrome

Extended X-Ray Absorption Fine Structure Spectroscopy (EXAFS)

C.S. Rossington in the 1-4 keV Region

60,900

K.M. Yu

W. Walukiewicz

J.M. Jaklevic

Environment, Health and Safety Division

R.-K.S. Sun Modification of an Andersson-Braun Type Remmeter to Extend the Sensitivity to $400 \mathrm{MeV}$

46,500

Information and Computing Science Division

J.F. Macfarlane Frameworks for Next-Generation Machining and Manufacturing

50.000

J.F. Macfarlane

POET: A Parallel Object-Oriented Environment and Toolkit

81,800

F. Olken

Robust Shotgun DNA Sequence Assembly

51,500

L.E. Sawler

D.M. Gusfield

\section{Life Sciences Division}

W.T. Chu

B.A. Ludewigt

Biophysical Modeling for Optimization Treatment Plans

94,900

T.M. Forte

Environmental Air Pollutants and Oxidative Stress

87,000

R.M. Krauss

T.L. Head-Gordon

Comprehensive Approach to Protein Folding

98,800 
Table 1. Continued.

M.F. Maestre

R.H. Fish

J.D. Rine

E.A. Ostrander

M.S. Roos

S.T.S. Wong

T.F. Budinger

Materials Sciences Division

A.P. Alivisatos

J. Bokor

E.D. Bourret

J. Amold

D.S. Chemla

J. Clarke

C.S. Fadley

E.E. Haller

W. Walukiewicz

P.L. McEuen

D.F. Ogletree

S. Weiss

J. Orenstein

G.D. Sucha

H.W.K. Tom
Organometallic Complexes as Novel DNA Sequencing Agents

50,000

Genetic Map of Canis Familiaris

100,000

Head Probe for Nuclear Magnetic Resonance Imaging and Spectroscopy at $10 \mathrm{~T}$

48,100

Valence-Band Photoemission from Semiconductor Nanocrystals

79,600

Electronic Thermalization in Metals and Semiconductors

180,900

MOCVD Growth and Characterization of II-VI Compounds

Using Non-Toxic Single-Molecule Precursors

75,000

Optical Investigations of Quantum Well Structures Under

High Magnetic Fields

Electron-Beam Lithographic Fabrication of Submicron Junctions for Macroscopic Quantum Tunneling, Coulomb Blockade and High- $T_{c}$ SQUIDS

Novel Surface and Interface Studies Using Photoelectron Spectroscopy Combined with Scanring Tunneling Microscopy

96,000

Isotope Heterostructures Selectively Doped by Neutron Transmutation

45,400

Electron Transport in Nanostructures

71,400

Near-Field Scanning Optical Microscopy/Spectroscopy of

Low-Dimensionality Materials at $50 \mathrm{~nm}$ Resolution

48,000

Quasiparticle Dynamics of High- $T_{C}$ Superconductors: Probing the Symmetry of the Pair Wavefunction

101,100

A Femtosecond Multi-Wavelength Source for Visible/Infrared Spectroscopy

Time-Resolved Studies of VUV, XUV, and Soft-X-Ray Photoinduced Chemistry of Surfaces 
Table 1. Continued.

Division Investigator

Project Title

(\$)

M.A. Van Hove

Picometer-Scale Structure of Interfaces from Synchrotron Radiation

133,000

C.S. Fadley

P.N. Ross

E.R. Weber

Scanning Tunneling Microscopy of Heterostructures and Interfaces in Cross Section

M.L. Cohen

New Fullerene-Based Materials

142,900

J.M. Hawkins

Y.-R. Shen

A. Zettl

Nuclear Sciences Division

S. Freedman

Laser Trapping of Neutral Radioactive Atoms

185,100

J.M. Nitschke

New Concepts in IsoSpin Studies

130,600

H.H. Wieman

Microstrip Gas Chambers for TPC Readout

103,700

W.G. Gong

J.W. Harris

J.T. Mitchell

\section{Physics Division}

G.S. Abrams

A. Ciocio

P. LeDu

The Portable DAQ: A Data Acquisition System for Particle Physics

Based on a Standardized Software Bus

149,600

A.R. Clark

F.R. Goozen

N. Roe

New Si Microstrip Disk Detector for Vertex Reconstruction

of B Meson Decays

135,000

B.A. Schumm

M.W. Strovink

G.F. Smoot

Directions for a Particle Astrophysics Initiative: Additional

Observations of the Cosmic Microwave Background

103,400

Structural Biology Division

S.R. Holbrook

Novel X-Ray Crystallographic Studies of RNA 


\section{Accelerator Fusion and Research Division}

\section{Beam Scanning: Modeling and System Specifications for an Integrated 3-D Proton Treatment Delivery System}

Principal Investigators: John W. Staples, Bernhard A. Ludewigt, and Jose R. Alonso

Funding: $\$ 49,800$

Project No. 93001

\section{Project Description}

The use of proton accelerators for radiotherapy allows full three-dimensional conformal treatment of a tumor volume by sweeping a pencil beam transversely and longitudinally, filling the volume to the desired dose. The technique of scanning irregular volumes is just now being developed and offers the potential of producing the best dose distribution in irregular volumes without the need for fixed mechanical collimators, scattering foils, or mechanical range shifters. Scanning the beam transversely by magnetic deflection and longitudinally by changing the accelerator energy allows optimal shaping of the dose distribution in the target volume and minimizing the dose outside it, reducing the complication rate.

The high-energy accelerator choice addressed in this study is the synchrotron, which has been used so far by LBL and by Loma Linda University Medical Center, among others. The synchrotron produces beams with easily variable intensity and energy but is also accompanied by other unwanted fluctuations in intensity, position, and energy. The main goal of this study was to determine how much fluctuation is permissible for a given error in the deposited dose distribution.

Accordingly, the application of a dose to a regular and an irregular volume with a three-dimensional conformal beam scanning delivery system was studied, and included various perturbations in intensity and position appropriate to typical beam characteristics generated by a synchrotron. A new and very effective beam scanning method was developed in the course of this study: namely, velocity scanning, which can cope with larger accelerator fluctuations than conventional constant-velocity beam scanning using intensity modulation and with inadequacies in the scanning system.

A specific purpose of this study was to determine operating parameters and tolerances and to derive accelerator.and beam delivery systems for a new proton radiotherapy facility.

\section{Accomplishments}

We modeled the dose deposition of a pencil beam of protons in uniform tissue. The scattering, straggling, and attenuation due to nuclear interactions was taken into account. An optimization procedure was used for determining the beam occupation function (defined below) that governs the duration the beam irradiates each voxel in the treatment volume. A simple rectangular volume and an irregular volume, representing thie tumor partially surrounding a sensitive organ, such as the spine or rectum, were modeled. Perturbations were applied to the accelerated beam, typical of these delivered by a. synchrotron.

The raster scanning method chosen uses velocity modulation instead of intensity modulation of the beam. This offers the following advantages:

- No modulation of the accelerator intensity is required.

- The good dynamic range provides the high occupation function values required at the edges of the dose volume.

- The nonperiodic line scan rate minimizes the effect of periodic ripple modulation of the beam intensity from the accelerator.

An optimization procedure is needed to determine how the target volume is best filled by pencil beams assuming no restrictions are imposed by the scanning system or the accelerator. Then limitations are applied, and the errors in the dose distribution are evaluated. The procedure consists of finding a function $F$ that delivers the required dose to the target volume while maximizing the lateral and distal falloffs. We have used an optimization procedure developed by Brahme and Lind. The function $F$ is approximated through an iteration process 


$$
\begin{gathered}
F_{0}=D_{0} \\
F_{n+1}=C\left[F_{n}+a\left(D_{0}-F_{n} \otimes P\right)\right] .
\end{gathered}
$$

Where $D_{0}$ is the desired dose distribution, $C$ is a constraint operator guaranteeing nonnegative occupation function amplitude, and $a$ is a convergence parameter.

The function $F$ is the irradiation or Bragg peak density defined throughout the volume and describes the amount of beam deposited in the volume with the center of the Bragg peak at a particular location.

Figure 1 shows the optimized Bragg peak density function on a plane perpendicular to the scan plane and through the central axis of the radiation field.

We have simulated the actual dose distribution with limited maximum slew rate of the sweep magnets and with realistic fluctuations in the accelerator beam intensity. The maximum sweep rate capability is limited to 10 times the average sweep rate. The perturbed dose distribution using $F$ was then calculated by imposing maximum slew rates on the scan velocity and introducing beam intensity fluctuations.

The accelerator beam intensity fluctuation is defined within a moving time window. Ripple, intensity spikes, and random variations are averaged within a moving time window. This reflects the integrating

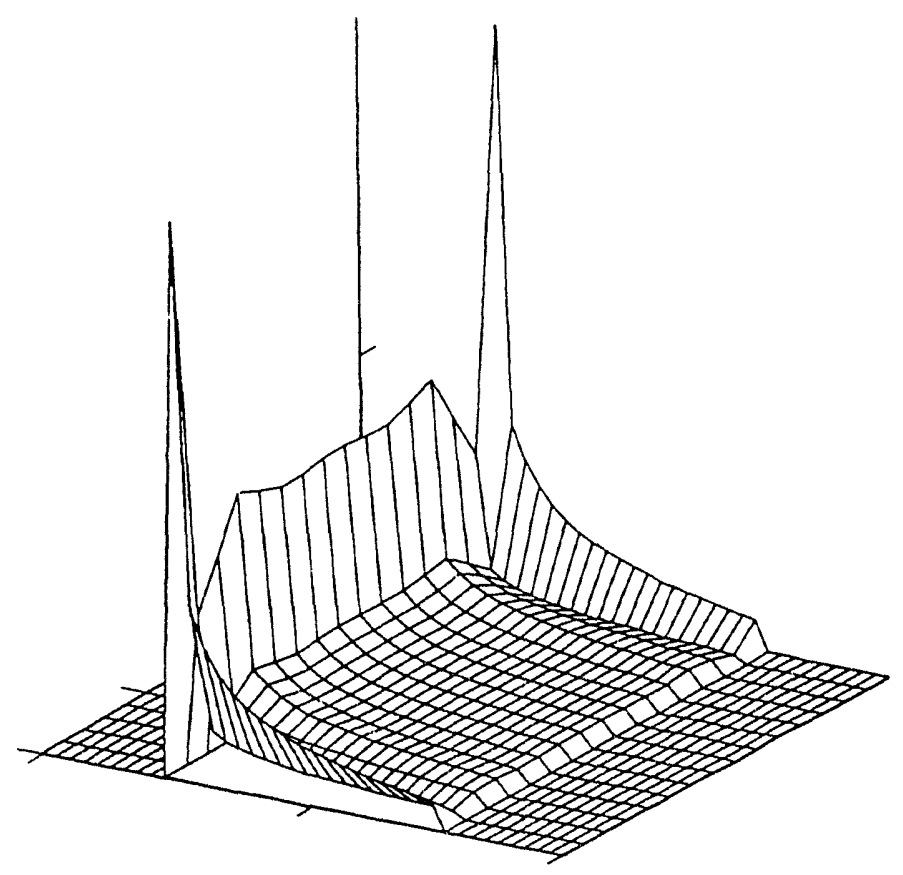

Fig. 1. Bragg peak density function $F$. effect of a finite beam width as it moves along its path. In our example, a beam with an rms size of 3$8 \mathrm{~mm}$ moves over a $1300-\mathrm{mm}$ path in one second. The

\begin{tabular}{|c|c|}
\hline Window & Fluctuation \\
\hline $200 \mu \mathrm{sec}$ & $\leq \pm 20 \%$ \\
\hline $100 \mu \mathrm{sec}$ & $\leq \pm 100 \%$ \\
\hline$<25 \mu$ sec & $\begin{array}{c}<5 \times 10^{6} \text { total particles } \\
\text { in } 25 \mu \mathrm{sec}\end{array}$ \\
\hline
\end{tabular}
maximum fluctuations within the window are:

Figure 2 shows the dose distribution for a $\pm 20 \%$ intensity fluctuation within a $200 \mu \mathrm{sec}$ traveling window, a scanner slew rate limitation of 10 times the average rate, and with an additional $180-\mathrm{Hz}$ ripple modulating the intensity by $30 \%$. This distribution differs by no more than $3 \%$ from a dose distribution with no perturbations and unlimited scanner slew rate.

Additional perturbations were applied to the beam and limitations applied to the maximum scanning rate. The table following lists the effect on the dose distribution in each of these cases. The fluctuation $\sigma$ defines the random intensity variations within a 25$\mu$ sec window. When $\sigma=0.35$, the $\pm 20 \%$ variation integrated in a $200 \mu \mathrm{sec}$ window is achieved.

The error indicated is the maximum deviation at any one point of the achieved dose distribution with the added fluctuations or scanner slew rate limitation from the distribution with no fluctuations.

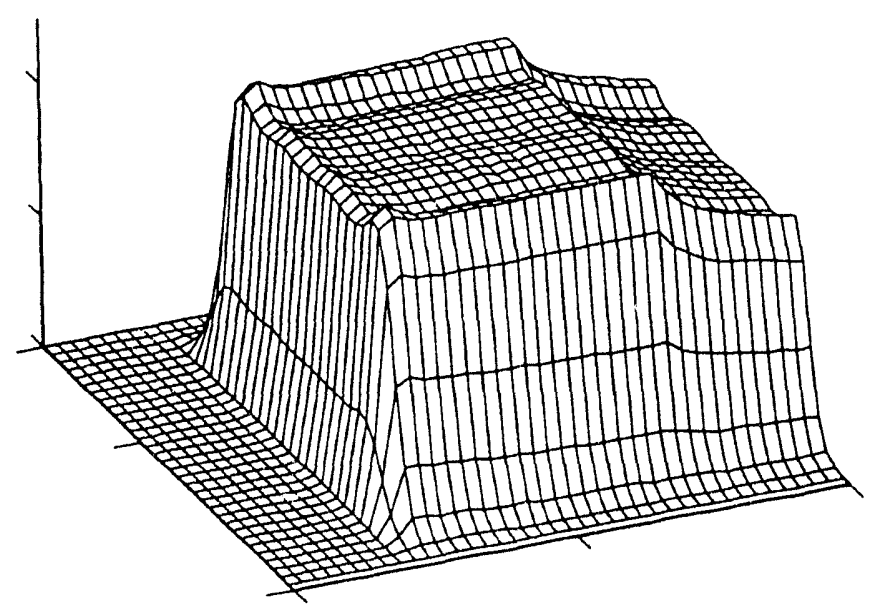

Fig. 2. Dose distribution with nominal perturbations. 


\begin{tabular}{lr}
\hline Simulation Parameter & Error \\
\hline Max sweep velocity = $2 \times$ average & $2 \%$ \\
Max sweep velocity = $1.2 \times$ average & $40 \%$ \\
Fluctuation $\sigma=0.35$ and $30 \% 180 \mathrm{~Hz}$ ripple & $3 \%$ \\
Fluctuation $\sigma=1.05$ and no a.c. ripple & $4 \%$ \\
Fluctuation $\sigma=0.35$ and $100 \% 180 \mathrm{~Hz}$ ripple & $7 \%$ \\
Fluctuation $\sigma=0.35$ and $30 \% 60 \mathrm{~Hz}$ ripple & $7 \%$ \\
Fluctuation $\sigma=0.35$ and $100 \% 60 \mathrm{~Hz}$ ripple & $19 \%$ \\
\hline
\end{tabular}

The results of this work have been essential for deriving the performance specifications for a proton radiotherapy facility published as an LBL document.

\section{Publications}

J.W. Staples and B.A. Ludewigt, "Modeling and System Specifications for an Integrated 3-D Proton Treatment Delivery System," to be published in Proceedings of the 1993 Particle Accelerator Conference, May 17-19, 1993, Washington, D.C.,

\section{Phase Equilibria and Microstructural Texture in Bi-oxide High- $T_{\mathcal{C}}$ Superconductors}

\author{
Principal Investigator: Daniel R. Dietderich \\ Funding: $\$ 50,800$ (FY 93) \\ $\$ 49,000$ (FY 92) \\ Project No. 92001
}

\section{Project Description}

The goal of this work is to gain a better understanding of the grain alignment mechanism in the $\mathrm{Bi}_{2} \mathrm{Sr}_{2} \mathrm{CaCu}_{2} \mathrm{O}_{8+\delta}$ compound (2212) and the special role of $\mathrm{Ag}$. Most of the previous work on dip-coat or doctor-blade processed 2212 has been empirical and focused on fabrication of conductors with high critical current densities. This project, however, addresses the fundamental factors affecting grain alignment, nucleation, and growth of the 2212 crystals. This work intends to provide an understanding of the mecha- nistic aspects of grain alignment. Tapes produced by the dip-coating process are advantageous for this investigation. Not only does the material produce a high $\mathrm{J}_{\mathrm{c}}$ when correctly processed but also the sample geometry with its free surface permits easy in situ and post heat-treatment characterization of samples; the process has the potential to produce long lengths of conductor at low cost. Better understanding of the grain alignment mechanism should lead to the fabrication of conductors with much higher critical currents and possibly better mechanical strength and fracture toughness.

\section{Accomplishments}

Some of the highest $\mathrm{J}_{\mathrm{c}}$ 's reported for thick films (Fig. 3) of $2212 \mathrm{Bi}$-oxide superconductors (greater than $10^{5} \mathrm{~A} / \mathrm{cm}^{2}$ at $4.2 \mathrm{~K}$ and $>6 \mathrm{~T}$ ) are for tapes produced by the dip-coating processes at LBL. This work has provided important information on the relationship between processing, microstructure, and properties. One is the effect of heat treatment schedule on the grain alignment of the microstructure and the other is the importance of an interface or surface to promote grain alignment. The optimum heat-treatment schedule, slowly cooling the material from the partially molten state, produces an aligned microstructure that significantly increased $J_{C}$. After melt processing the surface of the material can be highly textured even if the underlying material is not. The surface effect becomes even more pronounced in the absence of $\mathrm{Ag}$ and as the film thickness decreased.

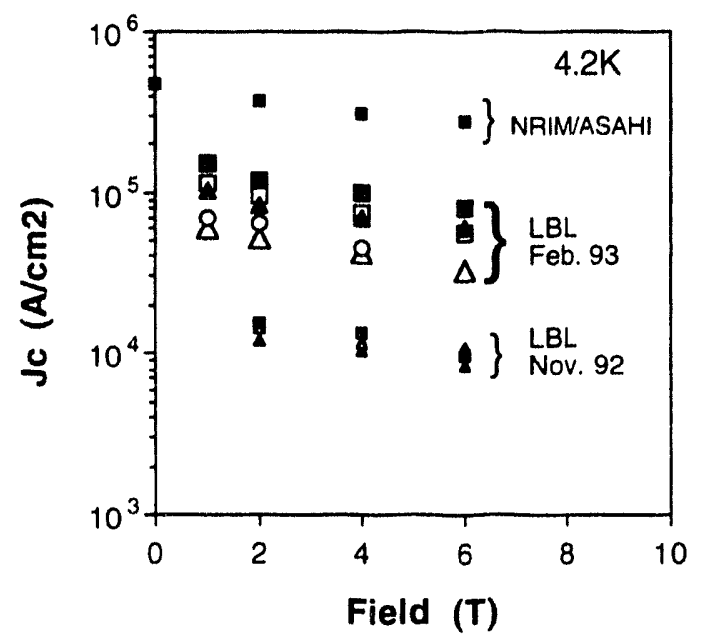

Fig. 3. LBL progress in improving the critical current density of short samples of 2212 dip-coated Ag tape, compared to the results of NRIM/Asahi Glass Inc. The different symbols for the LBL data are for various process conditions. 
The high-critical current density of the 2212 compound produced by the doctor-blade and dip-coating processes and the work being performed at LBL have sparked industrial interest, especially in studying the 2212 material and in developing a dip-coating process. It is felt that the work being done at LBL will complement, possibly even supersede, current programs on 2212 powder-in-a-tube process. The progress in increasing $\mathrm{J}_{\mathrm{c}}$ can be seen in Fig. 3 . The capability to fabricate uniform long lengths of material reproducibly at low cost now established at LBL will strongly complement our ongoing superconducting materials work and the fundamental investigations proposed for this study.

The LBL work is the first to document a grain alignment mechanism for the 2212 phase. Microstructural studies have shown that 2212 forms on cooling by a nucleation and growth process. Scanning electron microscopy (SEM) observations reveal that the 2212 crystals grow by a terrace-ledge-kink type mechanism. The nucleation site is still uncertain but the SEM image and transmission electron microscopy diffraction patterns show that the [010] and [100] directions are not equivalent due to an incommensurate modulation along [010] and that the fast growth direction is [100]. Controlling the distribution and possibly the orientation of the nucleation sites present in the liquid oxide at high temperature could produce a material with better grain alignment and a higher critical current.

\section{Scattering Techniques for Femtosecond X-ray Pulses}

Principal Investigator: Richard A. Gough

Funding: $\$ 477,700$

Project No. 93002

\section{Project Description}

The purpose of this project was to initiate an experimental investigation to test a novel scheme of generating, detecting, and eventually using as a scientific tool (to probe ultrafast phenomena) ultrashort $x$-ray pulses of femtoseconds duration based on a $90^{\circ}$ Thomson scattering technique. Femtosecond $x$-ray pulses are potentially important tools to study fast chemical reactions in a time-resolved manner and allows study of coherent intramolecular dynamics in chemistry and phonon-dynamics in the solid state. Such a tool is thus expected to open new avenues of investigation in condensed matter physics, chemistry, biochemistry, and all ultrafast phenomena.

Out of several possible approaches to generate short pulse $x$-rays, we have identified one very attractive and promising route that is simple in principle and takes full advantage of the recent progress in particle accelerator and laser techniques. This technique involves Thomson scattering a short laser pulse in the visible (few tens to few hundreds of femtoseconds (a) $0.8 \mu \mathrm{m}$ say) off a low-energy electron beam of normal duration $(\sim 10 \mathrm{ps})$ at right angles to each other. The idea is to utilize the short transverse dimension of a tightly focused low-emittance electron beam and an ultrashort pulse laser beam. The short pulse radiation is produced in the direction of the electron beam. Simple estimation shows that a $32 \mathrm{MeV}$ electron beam containing $1.6 \mu \mathrm{C}\left(10^{10}\right.$ electrons) of charge per $10 \mathrm{ps}$ bunch with a normalized emittance of $5 \mathrm{~mm}$-mrad, when focused down to $100 \mu \mathrm{m}(\approx 330 \mathrm{fs})$ could completely overlap transversely a $0.8 \mu \mathrm{m}, 330 \mathrm{fs}$ laser beam and scatter off to produce $1 \AA x$-ray bursts of $330 \mathrm{fs}$ duration containing $2.7 \times 10^{5}$ photons, if the laser intensity is of the order of $3 \times 10^{19} \mathrm{~W} / \mathrm{m}^{2}$.

We have begun preparations for performing a proofof-principle experiment of the $90^{\circ}$ Thomson scattering in the Beam Test Facility at the Advanced Light Source (ALS) injector, being constructed by the Center for Beam Physics.

\section{Accomplishments}

In FY 1993, we have achieved major progress in bringing this experiment closer to reality. The experiment will use the $20-50 \mathrm{MeV}$ electron beam of the ALS injector linac during 4-6 hours of storage time of the electron beam in the ALS main storage ring. The injector beam will be transferred to a specially shielded vault by a transport line designed with specific magnetic optics in mind.

The magnetic lattice that would be required to bring the electron beam at the collision point with proper transverse and temporal profiles has been worked out and incorporated into the design of the transport line being constructed in the Beam Test Facility. The tolerance on the magnetic fields and timing have been set and are being implemented. Electron beam diagnostics have been worked out and are being installed as part of the beam line. A special C-magnet to separate the electron beam from the $x$-ray beam 
and special beam dump shielding for screening the primary $x$-ray signal from background are being designed. The location of the Ti-Sapphire laser system on the roof block has been identified, surveyed, and approved.

Work continues on bringing utilities, etc., to this site. The laser system itself has been parameirized and designed. Major components have already been ordered. With the scheduled completion of the Beam Test Facility vault approaching, we are poised to begin installation of the laser, the scattering chamber and $x$-ray diagnostics in FY 1994 and perform the experiment.

\section{Publications}

K.-J. Kim, S. Chattopadhyay, and C.V. Shank, "Generation of Femtosecond X-rays by $90^{\circ}$ Thomson Scattering," to be published in Nucl. Instrum. Meth. in Phys. Res. as part of the Proceedings of the XV International Conf. on Free Electron Lasers, The Hague, Netherlands, August 1993; LBL-33074.

W. Leemans, G. Behrsing, K.-J. Kim, J. Krupnick, C. Matuk, F. Selph, and S. Chattopadhyay, "The $50 \mathrm{MeV}$ Beam Test Facility at LBL," and presented at the IEEE Particle Accelerator Conf., Washington, D.C., May 1720,1993, to be published in the Proceedings; LBL33308.

W. Leemans, E. Wallace, A.M. Sessler, and S. Chattopadhyay, "Status of the $50 \mathrm{MeV}$ Plasma Lens Experiments at LBL," presented at the 35th Annual Meeting, APS Division of Plasma Physics, St. Louis, MO, Nov. 1-5, 1993; LBL-34335 Abs.

\section{Study of Coherent Radiation Sources beyond ALS}

Principal Investigator: Ming Xie

Funding: $\$ 199,300$

Project No. 93003

\section{Project Description}

The principal long-range goal of this project is to conceptualize an advanced radiation facility beyond the ALS at Berkeley. Several crucial physical issues and key technical components will be studied for the generation of intense, coherent radiation. In particular, the emphasis will be placed on the short wavelengths of considerable interest for applications, such as soft $\mathrm{x}$-rays in the biological "water window" around $40 \AA$ for fundarnental research at the cutting edge of life sciences. The program of research will carry out this mission along the following broad fronts: (1) study of generation mechanisms, (2) fundamental characterization of radiation properties, (3) accelerator system $R \& D$, and (4) optical resonator R\&D.

\section{Accomplishments \\ Study of Generation Mechanisms}

Recent developments in linear accelerator technology make it possible to produce electron beams of sufficiently high brightness for an extremely-highgain free electron laser (FEL) amplifier. With such an amplifier, lasing can be achieved in a single pass through the self-amplified-spontaneous-emission (SASE), thus obviating the need for high reflectivity mirrors hardly available at short wavelengths for an FEL oscillator. It is then possible to generate coherent radiation for wavelengths shorter than $1000 \AA$ at a peak power of hundreds of megawatts to several gigawatts, comparing to 10 watts or so available from synchrotron radiation facilities.

We have carried out extensive analytical and numerical study of such an amplifier in both exponential growth and saturation regime, and extended our previous one-dimensional study of SASE to three-dimensional (3D). We have obtained the first exact 3D solution for the amplifier gain and FEL eigenmodes taking into account the effects of beam emittance, betatron focusing, and energy spread. We have also obtained through extensive simulations with 3D code TDA, a universal scaling law was also obtained, and an empirical formula for the saturation power. These studies have laid ground work for the fundamental understanding of the FEL startup and amplification process, and provided the efficient tools for the design and optimization of high gain FELs. These tools are used currently in the feasibility studies of an $x$-ray FEL at "water window" based on the existing linacs at Stanford Linear Accelerator Center, with which we have been actively collaborating.

\section{Fundamental Characterization of Radiation Properties}

Propagation of coherent radiation through optical elements cannot be completely described by geo- 
metric optics because of the wave nature of coherent radiation. We have initiated a basic study of propagation and characterization of optical coherence was initiated through the use of Wigner function associated with optical field, and successfully applied the method to the synchrotron radiation. For a deeper understanding of the role of the electron beam emittance on the coherence, an approach quantifying the relationship we have developed between the phase space area and the entropy of the radiation field. The method was applied to characterize the coherence of undulator radiation.

\section{Acrelerator System RED}

Laser driven radio-frequency ( $\mathrm{rf}$ ) photocathode holds great promise as a source of tightly bunched highbrightness electron beams. It is an important component for the generation of short wavelength radiation as well as for many accelerator applications.

Experimental characterization and optimization have been carried out with a barium photocathode and a nitrogen laser. The dependence of the quantum efficiency on the laser beam polarization and angle of incidence were measured. An electron quantum efficiency as high as $10^{-3}$ was observed.

To reduce the stringent emittance requirements for a FEL in short wavelengths, a new idea of conditioning the phase-space of an electron beam by a microwave cavity has been proposed recently. In attempting to build and test such a conditioner, possible designs for conditioning rf cavities have been studied analytically and numerically. The performance characteristics as a function of cavity parameters have been investigated.

Close attention was paid to the development of superconducting rf (SCRF) linear accelerator technology, as it offers the inherent advantage of intrinsically stable operation since it functions in the continuous wave mode. It is necessary to demonstrate that this technology is feasible also for high current operation as demanded by intense light sources. As part of Stanford/LBL /BNL collaboration to develop SCRF accelerator for FELs, we have tested the $500 \mathrm{MHz}$ SCRF cavity acquired from TRW at $4.5 \mathrm{~K}$ and a rf input matching network, measured the higher order modes (HOM) warm and cold, and investigated the effects of microphonics and the HOM couplers.

\section{Optical Resonator REDD}

For several years we have been developing a versatile simulation code, SWORD (Small Workshop for Optical Resonator Design), for design and optimization of generic optical resonators. A couple of years ago, the code was used as a prinary tool in the design of a hole-coupled FEL resonator for the proposed Chemical Dynamics Research Laboratory (CDRL) infrared free electron laser (IRFEL). Recently, the code was used as part of Continuous Electron Beam Accelerator Facility (CEBAF)/LBL collaboration in the design of a confocal ring resonator for the proposed CEBAF IRFEL. To bench-test the code we have also carried out a scaled resonator experiment utilizing a visible HeNe laser.

There has been a growing interest in developing a more compact, though not coherent, $x$-ray source through Compton scattering of a laser beam by a relativistic electron beam. To get higher $\mathrm{x}$-ray flux it is advantageous to arrange the scattering to occur inside an optical resonator where the laser is most intense. We have proposed and studied a novel resonator configuration that is optimized for this type of applications. The resonator, called a double-confocal resonator, is suitable for either a FEL laser or a conventional one.

\section{Publications}

K-J. Kim and M. Xie, "Self-Amplified Spontaneous Emission for Short Wavelength Coherent Radiation," Nucl. Instrum. Meth. in Phys. Res. A331, 359 (1993).

Y. Chin, K-J. Kim, and M. Xie, "Three-Dimensional Theory of the Small-Signal High-Gain Free Electron Laser Including Betatron Oscillations," Phys. Rev. A 46(10), 6662 (1992).

Y. Chin, K-J. Kim, and M. Xie, "Calculation of 3-D Free Electron Laser Gain: Comparison with Simulation and Generalization to Elliptical Cross Section," Nucl. Instrum. Meth. in Phys. Res. A331, 429 (1993).

Y. Chin, "Simple Formulae for the Optimization of the FEL Gain Length Including the Effects of Emittance, Betatron Oscillations and Energy Spread," Nucl. Instrum. Meth. in Phys. Res. A331, 424 (1993).

M. Xie, "Exact and Variational Calculations of Eigenmodes for Three-Dimensional FEL Interaction in the Exponential Gain Regime," in preparation.

H. Winick, K. Bane, R. Boyce, G. Loew, P. Morton, H.D. Nuhn, J. Paterson, P. Pianetta, J. Seeman, T. Raubenheimer, R. Tatchyn, V. Vylet, C. Pellegrini, J. Rosenzweig, G. Travish, D. Prosnitz, T. Scharlemann, K. Halbach, K.-J. Kim, M. Xie, "A 2-4 nm Linac Coherent Light Source (LCLS) Using the SLAC Linac," to be published in the proceedings of the 
Particle Accelerator Conference, May 17-19, 1993, Washington D.C.

K.-J. Kim, M. Xie, E. T. Scharlemann, C. Pellegrini, G. Travish, "Performance Characteristics, Optimization, and Error Tolerances of a $4 \mathrm{~nm}$ FEL Based on the SLAC Linac," to be published in the proceedings of the Particle Accelerator Conference, May 17-19, 1993, Washington D.C.

K.-J. Kim and R. Littlejohn, "Effect of Electron Beam on the Entropy and Phase Space Area in Undulator Radiation," in preparation.

M.E. Conde, S. Chattopadhyay, K.-J. Kim, S.-I. Kwon, K.-N. Leung, and A.T. Young, "Electron Quantum Yields from a Barium Photocathode Illuminaied with Polarized Light," to be published in the proceedings of the Particle Accelerator Conference, May 17-19, 1993, Washington D.C.; also LBL-33303.

R. Govil, R.A. Rimmer, A.M. Sessler and H.G. Kirk, "Design of RF Conditioner Cavities," Nucl. Instrum. Meth. in Phys. Res. A331, 335 (1993).

J. Edighoffer, "TRW's 2-cell Niobium $500 \mathrm{MHz}$ Cavity HOM Measurements," Draft CPB Tech Note, 1993.
J. Edighoffer and W. Barry, "Demonstration of RF Input Coupler and Impedance Matching Network for Superconducting FEL Applications," Draft CPB Tech Note, 1992.

M. Xie and K-J. Kim, "Hole-Coupled Resonators for Broadly Tunable Infrared Free Electron Lasers," Proceedings of SPIE Conference 1868, 150 (1993).

S. Krishnagopal, M. Xie, and K.-J. Kim, "Suppression of Mode-Beating in a Saturated Hole-Coupled FEL Oscillator," Nucl. Instrum. and Meth. in Phys. Res. A331, 645 (1993).

S. Benson, H. Liu, G. Neil, and M. Xie, "A Confocal Ring Resonator for the CEBAF Infrared Free Electron Laser," submitted to IEEE J. Quantum Electronics, 1993.

W. Leemans, M. Xie, J. Edighoffer, K-J. Kim, and S. Chattopadhyay, "Experiment and Simulation of HoleCoupled Resonator Modes with a CW HeNe Laser," Nucl. Instrum. Meth. in Phys. Res. A331, 630 (1993).

M. Xie and K.-J. Kim, "A Double-Confocal Resonators for X-Ray Generation via Intracavity Compton Scattering," in preparation. 


\section{Chemical Sciences Division}

\section{Exploration of the Interface between Homogeneous and Heterogeneous Catalysis: Synthesis and Study of Organometallic Catalysts Supported on Novel Polysiloxane Materials with Controllable Solubility Properties}

Principal Investigators: Robert $G$ Bergman, Bruce M. Novak, Tara Y. Meyer, and Joyce Yu

Funding: $\$ 118,900$ (FY 93)

Project No. 92002 $\$ 69,600$ (FY 92)

\section{Project Description}

Both homogeneous and heterogeneous catalysis are used by the chemical industry for conversion of feedstocks to commercial chemical products.

Heterogeneous catalysts offer the practical advantage of catalyst stability and ease of processing. However, the structures of heterogeneous catalysts and the mechanisms of their reactions cannot be studied easily, and therefore it has historically been difficult to design or modify them in a rational way.

Homogeneous catalysts are more difficult to use, but nevertheless are employed in several large-scale processes (e.g., hydroformylation, methanol carbonylation) because they provide selectivity or chemistry not available in heterogeneous systems. They are almost always more highly characterized than their heterogeneous counterparts, and the mechanisms of their reactions are better understood. As a result, homogeneous catalytic processes are typically more amenable to improvement by systematic structural modification.

In view of the complementary advantages of homogeneous and heterogeneous catalysts, for several years there has been intense interest in developing "hybrid" catalysts that combine the useful properties of both types of systems. An important approach to achieving this goal has been the development of systems in which well-characterized homogeneous catalysts are "heterogenized"-i.e., they are covalently attached to robust solid supports.
Silica gel is one of the most important and widelyused supports for organometallic catalysts. The goal of this project is the understanding of silica supportcatalyst interactions through a continuum approach, wherein the boundary between homogeneous and heterogeneous catalysis systems will be incrementally traversed.

\section{Accomplishments}

During FY 1992, the first year of this project, we prepared soluble polysiloxane supports that consisted of polysilicic acid oligomers in which the free silanols were partially capped with trimethylsilyl groups. These polysiloxane supports have now been treated with both zirconium and iridium substrates to produce bound-metal complexes. Consistent with observations in analogous heterogeneous systems, the zirconium substrates, $\mathrm{Zr}\left(\mathrm{CH}_{2} \mathrm{CMe}_{3}\right)_{4}, \mathrm{Zr}\left(\mathrm{CH}_{2} \mathrm{Ph}\right)_{4}$, $\mathrm{Cp}^{*} \mathrm{ZrNp}_{3}$, and $\mathrm{C}_{\mathrm{p}_{2}} \mathrm{ZrMe}_{2}$, bind to the polysiloxane supports by silanol protonation of one or more alkyl groups. The resulting polysiloxane-bound zirconium species are soluble in hydrocarbon and ethereal solvents, allowing them to be characterized by NMR spectroscopy. Activation of these catalysts by treatment with hydrogen resulted in gellation in the cases of $\mathrm{Zr}\left(\mathrm{CH}_{2} \mathrm{CMe}_{3}\right)_{4}$ and $\mathrm{Zr}\left(\mathrm{CH}_{2} \mathrm{Ph}\right)_{4}$, but the use of the more sterically demanding and less labile $\mathrm{Cp}^{*}$ ligand in the $\mathrm{Cp}^{*} \mathrm{ZrNp}_{3}$ substrate resolved this problem. Efforts are currently underway to characterize the resulting supported metal hydride complex and to investigate its catalytic activity.

Reaction of $\mathrm{Cp} * \operatorname{Ir}(\mathrm{OH})\left(\mathrm{PMe}_{3}\right)(\mathrm{Ph})$ with the soluble polysiloxane support produced the bound-metal complex, $\mathrm{Cp}^{*} \operatorname{Ir}\left(\mathrm{O}\right.$ siloxane)( $\left.\mathrm{PMe}_{3}\right)(\mathrm{Ph})$. In this case, the metal complex binds by exchange of the hydroxide for a silanol group. This class of ligand exchange was further exploited to cleave the iridium complex from the support by reaction with phenol. The isolation of the cleavage product, $\mathrm{Cp} * \mathrm{Ir}(\mathrm{OPh})\left(\mathrm{PMe}_{3}\right)(\mathrm{Ph})$, confirmed that the complex had not undergone significant structural changes upon binding.

The ability to remove a metal complex from a support has direct application to the analogous heterogeneous systems. A complementary study of the chemistry of the $\mathrm{CP}^{*} \operatorname{Ir}(\mathrm{OR})\left(\mathrm{PMe}_{3}\right)(\mathrm{Ph})\left(\mathrm{R}=\mathrm{H}, \mathrm{OSiMe}^{\mathrm{t}} \mathrm{Bu}\right)$ on silica 
has thus been undertaken. As in the case of the soluble polysiloxane supports, the iridium complex can be bound and then removed by exchange with phenol. The supported complex also undergoes clean stoichiometric chemistry with organic reagents. For example, on treatment with acetylene dicarboxylate the supported iridium complex reacts and then releases an organic product with a new metallacyclic ring. This chemistry is summarized in Fig. 1. Thus the starting iridium complexes can be (1) supported on silica, (2) removed in intact molecular form and fully characterized, and (3) converted to a new supported complex that can be removed in intact molecular form and characterized. To our knowledge this is the first time that it has been possible to utilize the polymeric silica support as an apparently simple and detachable ligand in an organometallic transformation. In addition, in the phenol reaction it appears that substituted phenols with different acidities $\left(\mathrm{pK}_{\mathrm{a}}\right.$ 's) remove different amounts of the supported iridium complex from the silica. We are currently investigating this unique dependence of the extent of reaction on the $\mathrm{pK}_{\mathrm{a}}$ of the substituted phenol. Preliminary data suggest that this reaction may be useful for mapping silica sites with differing iridium affinities.

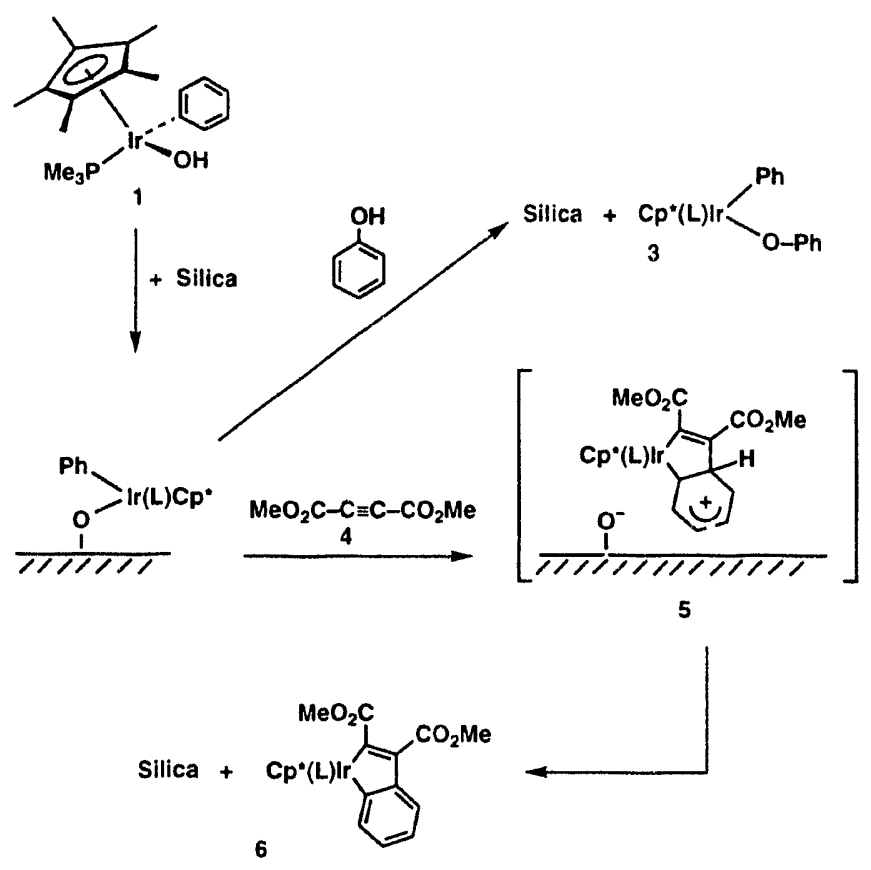

Fig. 1. First observation of reversible and controllable chemisorption of an organometallic complex to a silica support.
Publication

T.Y. Meyer, K.A. Woerpel, R.G. Bergman, and B.M. Novak, "Silica as a Ligand for Transition Metal Centers: Reversible Attachment and Displacement of Iridium(I) from a Silica Support," in preparation.

\section{Ultrafast Dynamics of Electrons at Surfaces}

Principal Investigator: Charles B. Harris

Funding: $\$ 100,400$ (FY 93)

Project No. 92003 $\$ 125,400$ (FY 92)

\section{Project Description}

Techniques are being developed to examine the spectroscopy and dynamics of surface electronic excitations at interfaces with unprecedented precision. Using angle-resolved two-photon photoemission, one can study the band structure of electronically excited states at surfaces. The use of fiber-optic/grating-pair pulse compression with a picosecond laser system makes possible direct measurement of excited state lifetimes on the femtosecond time scale. This provides a unique ability to explore excited state dynamics of electrons at metal-dielectric interfaces.

The electronic dynamics of surfaces are involved in many problems of fundamental interest from twodimensional magnetism to chemisorption. Directly related areas of technological significance include surface photochemistry, laser-surface interactions such as damage mechanisms in optics, surface recombination that can dominate carrier lifetimes at semiconductor interfaces, and magnetic data storage devices. A surface electronic state is characterized not only by its energy, but also by its momentum parallel to the surface. Energy transfer between states requires that both these quantities be conserved. Thus a complete understanding of excited state dynamics requires time, energy, and momentum resolution.

The conventional means of determining the band structure of excited surface electronic states is kresolved inverse photoemission spectroscopy (KRIPES). This technique has been very successful, but has limited energy resolution and cannot be time- 
resolved. Scanning tunneling spectroscopy provides information on unoccupied energy levels with atomic resolution, but also has limited energy resolution and cannot provide time or momentum resolution (except in a few very special cases not of interest to chemistry). The newer method of two-photon photoemission (TPPE) has an order of magnitude better energy resolution than either of the aforementioned techniques and the potential to directly measure lifetimes.

An unusual electron time-of-flight detection scheme is employed, which gives high sensitivity and extreme precision in energy measurements. Samples are mounted on a goniometer angle-drive mechanism that was designed and built in our laboratory and is superior to commercially available systems. This allows parallel momentum measurements and the determination of band structure. Liquid helium cooling of the sample to $45 \mathrm{~K}$ enables study of a wide variety of physisorbed and chemisorbed species. Ultrafast time resolution makes this the most sophisticated system of its kind.

\section{Accomplishments}

A very significant discovery within the past year has been the observation of 2-D localization of electrons at metal-alkane interfaces as a nondispersive feature in angle-resolved TPPE. This represents the development of a new tool to study the spatial extent of interfacial electronic states. Our method is a unique and important tool for the study of Anderson localization because it measures the energy spectrum of the localized states and can be applied to atomically thin samples. Generally, we find that all aspherical alkanes support localized electronic states at the interface, while the spherical neopentane does not. Figure 2 shows the dispersion data as a function of angle for bilayers of $n$-pentane and neopentane. We may predict that similar localized states exist at metalpolymer interfaces and contribute to their electronic properties.

A systematic study of physisorbed metal-alkane interfaces has been completed. First, the sensitivity of TPPE to various adsorption phenomenta was extensively investigated. We have shown that TPPE is extremely sensitive to layer thickness at the level of a few percent of a monolayer. TPPE data for the image potential state in the presence of layers of $n$-pentane and neopentane are shown in Fig. 3. We are able to distinguish between adlayer growth modes and map out surface phase diagrams. We have catalogued the distinct surface spectra characteristic of a 2-D gas layer, 2-D solid layer, island formation, and clustering
Electron Dispersion for Alkane/Ag(111) Bilayers
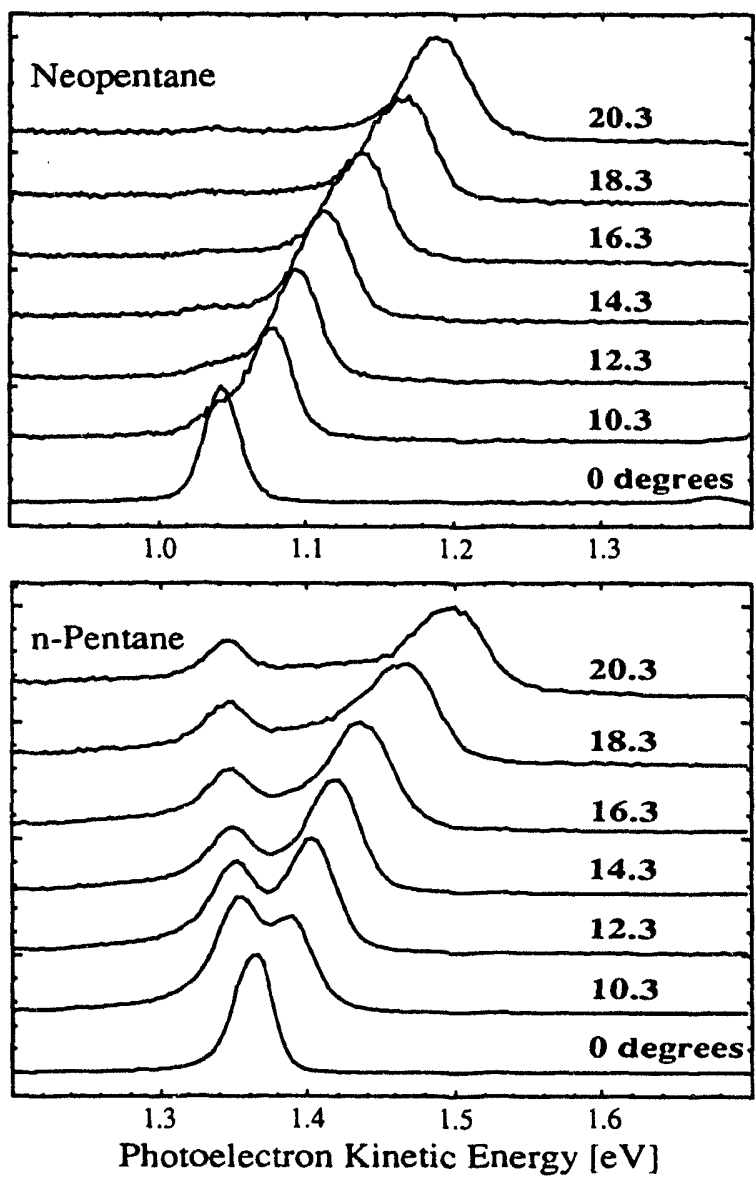

Fig. 2. A nondispersive feature (interpreted as a loculized electron state) is prominent for the n-pentane bilayer in the bottom frame, while the bilayer of neopentane is dominated by a parabolically dispersing band (extended electron state) of effective mass ratio $1.2 \pm 0.1$ in the top frame.

of a nonwetting adsorbate. Secondly, the dielectric properties of atomically thin slabs have been studied by measuring the effect of layer thickness on image potential electron binding energy. These layer-bylayer shifts are due to dielectric screening of the image potential and show how the atomic polarizability develops into the bulk dielectric constant. Thirdly, dispersion measurements of the image electron band structure have shown that the effect of the monolayer is to decouple the in-plane motion of the image potential electron from the metal bulk band structure, leaving it as a free electron in 2-D. However, the image electron effective mass increases for alkane multilayers due to small polaron formation.

Alkali layers on noble metals have been investigated both experimentally and theoretically. A TPPE study 
Layer-Resolved TPPE for Alkane/Ag(111)
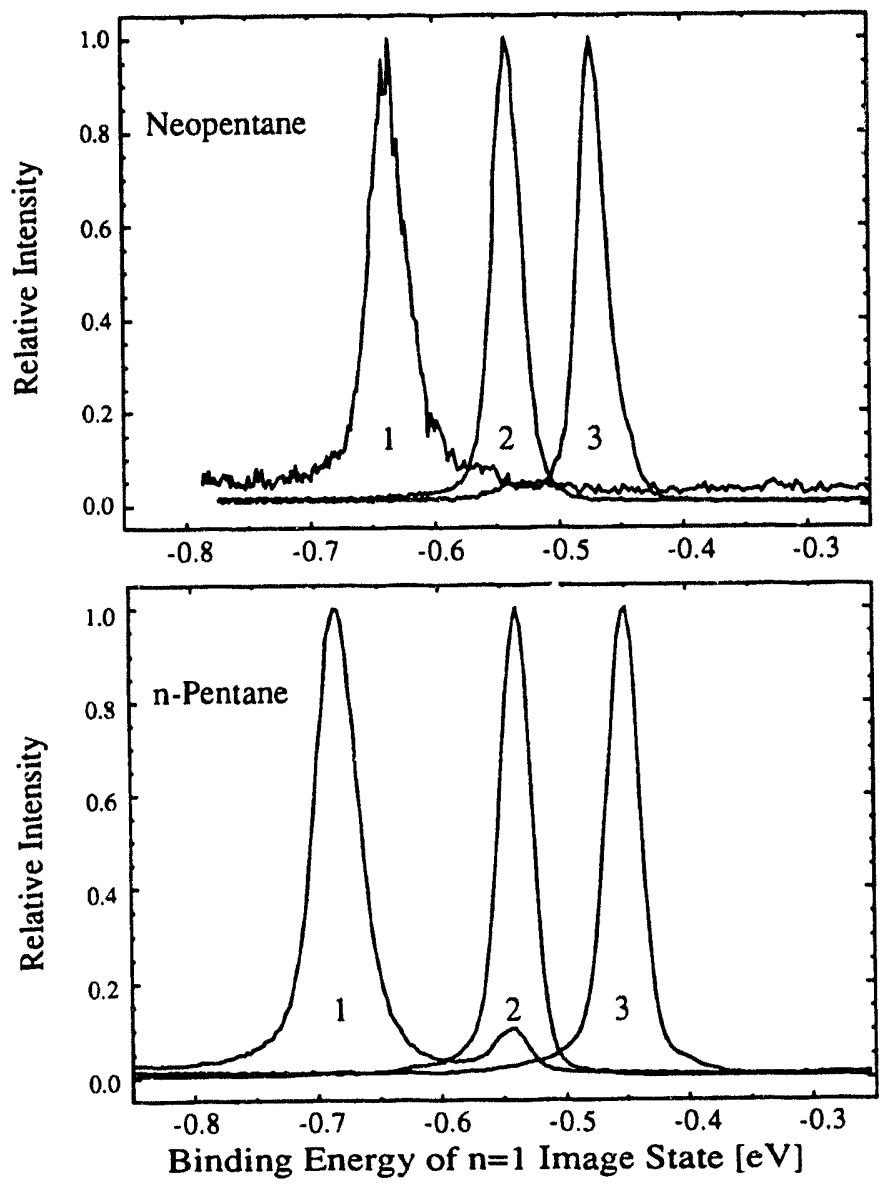

Fig. 3. Peaks in the TPPE spectrum shift to smaller binding energy as successive layers of dielectric material are adsorbed. The image state binding energy on bare $\mathrm{Ag}(111)$ is $-0.77 \mathrm{eV}$. The monolayer trace in the bottom frame shows small patches of bilayer, illustrating the insitu monitoring of layer growth.

of $\mathrm{K} / \mathrm{Ag}(111)$ found that the effective mass of the image potential electron is in relatively good agreement with predictions of a density functional calculation. The binding energy of image potential electrons on alkali layers has been successfully modeled using multiple reflection theory, contrary to results obtained by other workers. We conclude that multiple reflection theory is more robust than previously believed.

We have extended our previously reported study of the electronic structure of rare-gas films and have shown that dramatically different results are obtained for the bilayer compared to the monolayer. These results likely indicate the initial stages of the development of the band structure of the rare-gas solid. From this and from the alkane studies we conclude that most physisorbed monolayers behave similarly, excluding the image electron from the adlayer and decoupling its surface-parallel motion from the influence of the metal band structure. Binding energy and dispersion effects unique to a particular adsorbate species appear for the bilayer.

Chemisorbed methyl thiolate and sulfur on $\mathrm{Ag}(111)$ have been preliminarily studied; features are observed that are apparently not derived from the image potential state. These features are probably due to localized unoccupied electronic states associated with the chemisorption bond. Laser modifications are being made to enable the study of these very interesting and technologically relevant interfaces.

\section{Publications}

D.F. Padowitz, W.R. Merry, R.E. Jordan, and C.B. Harris, "Two-Photon Photoemission as a Probe of Electron Interactions with Atomically Thin Dielectric Films on Metal Surfaces," Phys. Rev. Lett. 69, 3583 (1992).

W.R. Merry, R.E. Jordan, D.F. Padowitz, and C.B. Harris, "Electrons at Metal-Insulator Interfaces. I. The Effect of Xe Monolayer on the Image Potential States of $\mathrm{Ag}(111), "$ Surf. Sci. 295, 393 (1993).

J.D. McNeill and C.B. Harris, "An Exact Phase Shift Model for Image States on Metal Surfaces with Alkali Metal Overlayers," Solid State Comm. 87, 1089 (1993).

Robert Lingle, Jr., D.F. Padowitz, R.E. Jordan, J.D. McNeill, and C.B. Harris, "Localization of Electrons at Interfaces," Proceedings of the 26th Jerusalem Symposium on Quantum Chemistry and Biochemistry, J. Jortner and B. Pullman, eds., Kluwer Academic Press, Dordrecht, The Netherlands, 1993.

R.L. Lingle, Jr., D.F. Padowitz, R.E. Jordan, J.D. McNeill, and C.B. Harris, "Two-Dimensional Localization of Electrons at Interfaces," submitted to Phys. Rev. Lett.

R.L. Lingle, Jr., J.D. McNeill, R.E. Jordan, D.F. Padowitz, and C.B. Harris, "Phase Transitions in Two-Dimensional Systems Studied by Two-Photon Photoemission," to be submitted to Surf. Sci. Lett.

R.E. Jordan, R.L. Lingle, Jr., J.D. McNeill, D.F. Padowitz, and C.B. Harris, "Electrons at MetalInsulator Interfaces. Il: Adsorbate Morphology and Electronic Structure on Alkane Multilayers," to be submitted to Surf. Sci. 
Structure and Chemistry of

Nonmetallic Adsorbates at Compound

Semiconductor Interfaces

Investigated by Synchrotron

Radiation Techniques

Principal Investigator: David K. Shuh

Funding: $\$ 76,100$

Project No.: 93004

\section{Project Description}

This research project emphasizes the investigation of the surface structures and chemistries of simple, nonmetallic adsorbates on compound semiconductor surfaces utilizing two complementary synchrotron radiation (SR) techniques. The experimental results provide insights on the fundamental interactions of chemisorption on binary semiconductors that are important in semiconductor processing technologies. A prime consideration is the application of SR approaches for characterization to make extensive use of the Advanced Light Source (ALS) capabilities.

The SR-based research relies on the emerging $x$-ray standing wave (XSW) technique for the determinations of adsorbate-semiconductor surface structures, whereas the more conventional methodology of synchrotron photoelectron spectroscopy (PES) has been utilized to characterize the chemical behavior of the systems under investigation. The XSW and PES experiments employ the existing endstations of the $x$ ray and vacuum ultraviolet electron storage rings of the Stanford Synchrotron Radiation Laboratory (SSRL) and the National Synchrotron Light Source (NSLS), prior to commencement of these activities at the ALS. Concomitant with the initial SR-based efforts, an ultra-high vacuum (UHV) surface science chamber with limited capabilities has been designed and constructed to support the initial SR projects. This small surface science system will permit testing of experimental apparatus under UHV and enable preliminary research investigations on the selected adsorbate-semiconductor systems.

\section{Accomplishments}

The prototype iodine/GaAs(110) interface investigated by $P E S$ and XSW techniques. The iodine on $\mathrm{GaAs}(110)$ system at room temperature is a particularly interesting system since I forms an ideal termination of the bulk $\mathrm{GaAs}(110)$ structure, from both chemical and structural perspectives. The I/GaAs interface orders and forms a near monolayer coverage. The initial PES and XSIA/ experiments were performed at SSRL X-ray Beamline 3-3 (Jumbo). The iodine was dispersed under UHV by a solid state electrochemical cell source, previously fabricated and tested at LBL. The iodine coverage and a suitable I detection feature were characterized by PES at the photon energy required to perform the XSW measurements, as shown in Fig. 4. The XSW data presented in Fig. 5 were collected by sweeping the photon energy through the Bragg condition while simultaneously monitoring the photoelectron signals from both the $I 3 p_{3} / 2$ peak and background region in the Constant Initial State (CIS) mode, then subtracting the background to extract the XSW signal from the iodine atoms.

The summed I XSW profiles (a single sweep is shown in Fig. $5 c$ ) are in the process of being fit to the dynamical x-ray theory of diffraction in order to extract the structural information from the I/GaAs(110) interface. In addition to the 220 reflection that yields the vertical distance of the I in relation to the GaAs $\{110\}$, the two distinct 111 reflections that lie approximately $\pm 35^{\circ}$ from the 220, were also measured to triangulate the absolute position of the I atoms on $\mathrm{GaAs}(110)$. Preliminary interpretation of the I XSW response suggests that iodine chemisorption is coherent and similar to that of $\mathrm{Sb}$ on the $\mathrm{GaAs}(110)$.

The design and fabrication of a simple surface science chamber system. To complement the SR program and

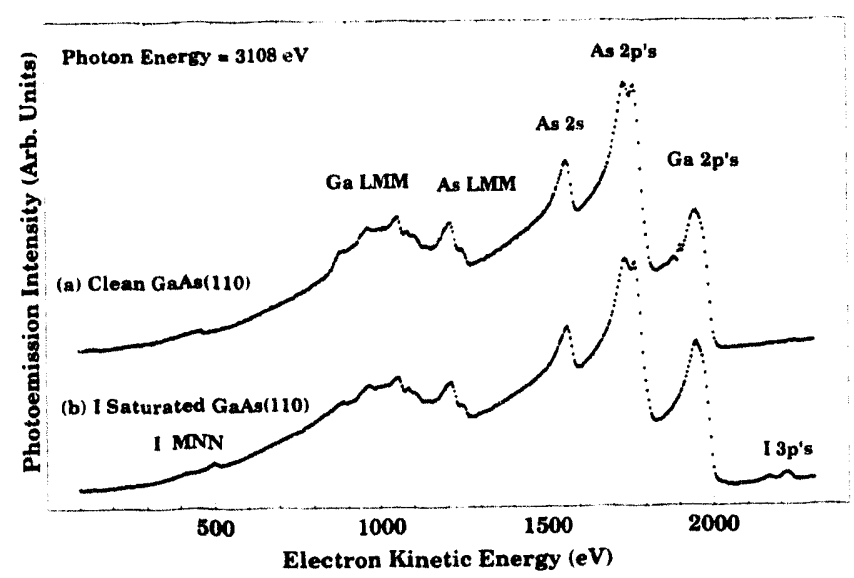

Fig. 4. Synchrotron PES spectra showing both the (a) clean and $(b)$ iodine saturated-GaAs(110). The PES spectra were collected with a double pass cylindrical mirror electron energy analyzer at a photon energy of $3108 \mathrm{eV}$, which corresponds to the Bragg condition for the GaAs 220 reflection used for the XSW measurements. 


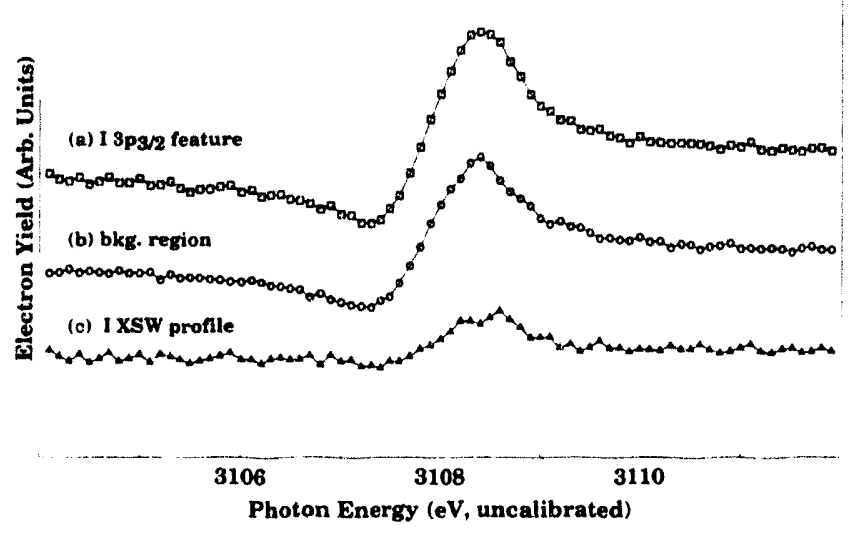

Fig. 5. The XSW GaAs 220 back reflection component spectra from a single CIS measurement illustrating the signals from the (a) I $3 p_{3 / 2}$ feature, (b) the background component taken $50 \mathrm{eV}$ higher than in (a), and the XSW profile that results from subtraction of (b) from (a). to enhance the productivity of the SR-based efforts, a modest laboratory-based UHV system was designed and constructed. The system allows the testing of experimental apparatus as well as the preliminary investigations of adsorbate-semiconductor experimental systems that are essential for successful experimentation at SR sources. The surface science chamber system provides the laboratory effort with the basic capabilities required to initiate and pursue a SR research program. The capabilities of the system can be increased and the chamber can, with further enhancements, serve as a simple SR endstation.

\section{Publications}

D. K. Shuh, T. Kendelewicz, and P. Pianetta, "Structure of the I/GaAs(100) Interface by X-SW," in preparation. 


\section{Earth Sciences Division}

\section{Exploratory Research on Seismic Imaging of Organic Liquid Contaminants in Unconsolidated Media}

Principal Investigators: Jil T. Geller and Larry R. Myer

Funding: $\$ 86,000$

Project No. 93005

\section{Project Description}

Subsurface contamination by organic liquids is ubiquitous. Owing to their low aqueous solubility, these contaminants migrate through and become entrapped in soils and aquifers as nonaqueous phase liquids (NAPLs). The partitioning of the trapped organic liquid into the aqueous and vapor phases contaminates underground water supplies. NAPLs migrate through preferred flow paths of least resistance and attain highly irregular distributions as residual segments break off from the bulk liquid and are held in pore spaces. The NAPL may pool over regions of low permeability.

Mapping the distribution of NAPL contaminants in the subsurface is critical to effective site remediation. Characterizing contamination from borehole samples covers a limited volume and due to the NAPLs irregular distribution, boreholes may miss the NAPL itself or create new contaminant pathways. In this project, a laboratory investigation was conducted to measure the response of ultrasonic waves to the presence of NAPLs in sand columns as a fundamental step towards the application of cross-hole seismic tomography to map NAPL distribution between boreholes and monitor changes during remediation. Seismic wave transmission through porous media is affected by the physical and chemical characteristics of the pore-filling fluid and its interaction with the solid phase. The differences in acoustic velocity, density, and viscosity between water and examples of NAPL contaminants, shown in Table 1, suggest that the seismic wave signature could indicate the presence of an immiscible organic liquid in unconsolidated porous media. The presence of interfaces between the immiscible liquids may also cause increased attenuation of energy compared to the porous media saturated by a single liquid phase.

The experimental apparatus consists of a triaxial test cell to control the confining and axial stresses on the sample. The endcaps that hold the sample contain piezoelectric crystals that transmit and receive compressional waves ( $p$-waves) by applying a voltage pulse across the crystal. The endcaps also have flow ports to introduce liquids and pressure ports for measuring sample permeability. A predetermined mass of quartz sand is packed into the Teflon sleeve held between the endplates to achieve a reproducible porosity between samples. The sample is saturated with water which is then displaced by injecting a NAPL. Received p-waves are recorded following incremental volumes of injected NAPL until water is no longer displaced. Similar experiments are conducted with initially NAPL-saturated media which is displaced by water.

Table 1. Acoustic velocities and physical properties of NAPL contaminants. ${ }^{a}$

\begin{tabular}{lllcl}
\hline Liquid & $\begin{array}{c}\text { Velocity } \\
\left(\mathrm{km} \mathrm{s}^{-1}\right)\end{array}$ & $\begin{array}{c}\text { Density } \\
\left(\mathrm{kg} \mathrm{m}^{-3}\right)\end{array}$ & $\begin{array}{c}\text { Liquid Bulk } \\
\text { Modulus } \\
(\mathrm{MPa})\end{array}$ & $\begin{array}{c}\text { Viscosity } \\
(\mathrm{mPa} \mathrm{s})\end{array}$ \\
\hline Water & 1.497 & 997 & 2234 & 0.890 \\
$\mathrm{n}$-dodecane & $1.29\left(22^{\circ} \mathrm{C}\right)$ & 745 & 1240 & 1.378 \\
$2,2,4$ trimethyl pentane (iso-octane) & $1.12\left(24^{\circ} \mathrm{C}\right)$ & $690\left(20^{\circ} \mathrm{C}\right)$ & 866 & $0.504\left(20^{\circ} \mathrm{C}\right)$ \\
trichlorotrifluoroethane (freon 113) & 0.716 & 1560 & 800 & $0.711\left(20^{\circ} \mathrm{C}\right)$ \\
\hline
\end{tabular}

avalues were obtained from the literature references available from authors upon request.

Temperatures are $25^{\circ} \mathrm{C}$, unless otherwise noted. 


\section{Accomplishments}

Our measurements have demonstrated the sensitivity of $p$-wave velocity and the attenuation of energy to the presence of different NAPLs. Data for tests conducted with n-dodecane, iso-octane, and freon 113 are shown in Fig. 1, which plots the p-wave velocity as a function of water saturation $\left(\mathrm{S}_{\mathrm{w}}\right)$, which is the fraction of water that occupies the pore space. The saturation is calculated from a mass balance of the NAPL injected into the column and collected in the effluent; therefore, it represents an average over the entire sample volume. Velocity decreases almost linearly as $\mathrm{S}_{w}$ decreases, until the residual saturation is attained. The filled symbols at zero water saturation are measurements made for initially NAPL-saturated sand packs. The magnitude of these values rank with the bulk moduli listed in Table 1 and fall on the same line as the measurements made with the initially water-saturated media. The data suggest that the velocity represents the average of wave propagation through the NAPL and water and are reasonably predicted by an analytical model by Kuster and Toksöz. In this model, the sample is represented as a composite liquid with solid spherical inclusions (sand grains). The physiral properties of the liquid are determined from the weighted average of the NAPL and water bulk moduli and densities.

The amplitude of the arriving wave changes significantly with the introduction of different volumes of NAPL. The data in Fig. 2, normalized by the amplitude for the initially water-saturated sample,

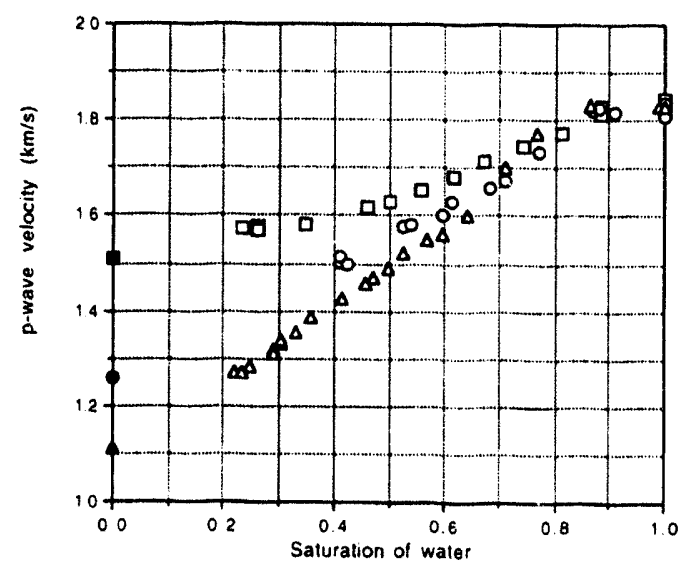

Fig. 1. P-wave velocity through sand samples as a function of water saturation. The remaining fraction of pore space is occupied by NAPL. $\square \mathbf{\square}=n$-dodecane, $0 \bullet=i$ iso-octane, $\Delta \Delta=$ freon 113.

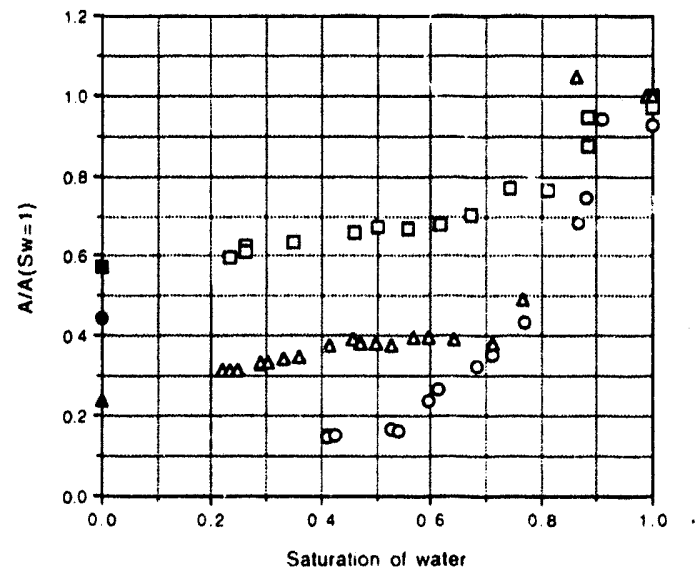

Fig. 2. Amplitude of first arrival (peak to peak) normalized by amplitude for water-saturated sand as a function of water saturation. $\square \mathbf{\square}=n$-dodecane, $\mathrm{O} \bullet=$ iso-octane, $\Delta \Delta=$ freon 113 .

indicate a more complex relationship than for velocity. The normalized amplitudes for the NAPLsaturated samples, which are the filled symbols, decrease in the order of n-dodecane, iso-octane, and freon 113, which is the same ranking as their bulk moduli. However, the minimum value for iso-octane at $S_{w}$ equal to 0.45 is much lower than for the other two NAPLs. The data may be explained by considering the effect of NAPL viscosity on its distribution within the sample for a given value of $\mathrm{S}_{\mathrm{w}}$. Iso-octane has the lowest viscosity of the three NAPLs. The displacement of a liquid by a less viscous one is unstable and may result in the formation of NAPL fingers extending the length of the sample. The partitioning or scattering of energy between the fingers may be the cause of the lower amplitudes observed in the experiment conducted with iso-octane.

The results of this investigation provide a basis for the application of high-frequency seismic tomography to detect NAPL contamination and indicate further research challenges. In heterogeneous natural formations, the seismic response may be nonunique. Nonetheless, seismic methods should be able to detect changes in NAPL distribution during remediation. The power of seismic methods for initial site characterization could be greatly enhanced by integrating other measurement techniques to constrain the nonunique response in heterogeneous media. 


\section{Publications}

J.T. Geller and L.R. Myer, "Seismic Imaging of Organic Liquid Contaminants in Unconsolidated Media," EOS Transactions, Am. Geophys. Union 74(43), 318 (1993).

\section{$X$-Ray Absorption Spectroscopy and $X$-Ray Microprobe Studies of Contaminated Soils}

Principal Investigator: Tetsu K. Tokunaga

Funding: $\$ 70,000$

Project No. 93006

\section{Project Description}

The possibilities for using synchrotron radiation sources such as the Advanced Light Source for gaining fundamertal insights into the distribution, speciation, and surface associations of contaminants in soils were the objectives of this research project. As a specific case for investigation, a study of selenium (Se) contaminated soils appeared attractive for numerous reasons. The importance of understanding the behavior of Se as a potentially toxic trace element in many soils has been well documented, and has received considerable recent attention following the discovery of Se contamination at Kesterson Reservoir, formerly part of the Kesterson National Wildlife Refuge, California. The critical role of the oxidationreduction status of Se and the specific nature of Se association with various solid phases are also well recognized. Such information is essential for understanding the stability, transformations, and mobility of Se in the environment.

Two promising methods of Se speciation of relatively undisturbed soil environments are extended x-ray absorption fine structure spectroscopy (EXAFS) and $x$-ray absorption near-edge structure spectroscopy (XANES). Both of these methods of $x$-ray absorption spectroscopy (XAS) require high-intensity synchrotron radiation. Successful applications of synchrotron radiation sources for XANES and EXAFS analyses on relatively undisturbed mineral specimens have recently elucidated the nature of surface complexation for a variety of trace elements including
Se. These past studies provided the first definitive characterizations of Se(IV) adsorption on mineral surfaces.

Another powerful synchrotron-based analytical method is that of the synchrotron $x$-ray fluorescence microprobe (SXRFM). The highly collimated, intense synchrotron radiation emitted from bending magnets is focused onto a small spot on a sample of interest, and the fluorescence spectrum is collected for analysis of elemental composition. The sample is sequentially scanned in front of the stationary $x$-ray beam, thereby permitting high-resolution, two-dimensional mapping of a range of elements. A number of scientific and technical issues require such fine-scale spatial resolution of chemical composition. There is an emerging awareness that such information can be essential in understanding the nature of contaminant transport in soil and rock systems. For the present study, the SXRFM was used primarily to map Se in soil systems.

\section{Accomplishments}

\section{Synchrotron X-Ray Absorption Spectroscopy Studies}

Selenium in contaminated soils from Kesterson Reservoir, California, and in waters and sediments from laboratory simulations of the contamination process was speciated using $x$-ray absorption spectroscopy. The XAS performed at the Stanford Synchrotron Radiation Laboratory in collaboration with Gordon E. Brown, Jr. and Ingrid Pickering, provided some of the first direct evidence for the occurrence of two species of Se in contaminated samples from Kesterson Reservoir. XAS of surface soils ( 0 to $0.10 \mathrm{~m}$ depth) in a formerly Typha-vegetated pond proved that elemental selenium [Se(0)] accounted for over $90 \%$ of the total Se in these samples. Previously, such speciation was inferred from analyses based on sequential chemical extraction methods. XAS performed on a mushroom (Agaricus bernardii) sample from Kesterson Reservoir was indicative of a selenium nearest-neighbor structure similar to that of seleno-methionine.

Samples from a laboratory experiment designed to reproduce features of the sediment contamination process were also analyzed with XAS. A solution containing $240 \mathrm{mg} \mathrm{l}^{-1}$ selenium as $\mathrm{Se}(\mathrm{VI})$, and with its major ion composition similar to that of Western San Joaquin Valley agricultural drainage waters, was ponded over previously uncontaminated soils collected from an area adjacent to Kesterson Reservoir. Sediments were analyzed at successive 
stages of ponding with XAS. These $x$-ray absorption spectra exhibited shifts in K-edges that demonstrate the sequential reduction of $\mathrm{Se}(\mathrm{VI})$ to $\mathrm{Se}(\mathrm{IV})$, and finally to $\mathrm{Se}(0)$. The evolution of the Se K-absorption edge spectra from these sediments, collected at various times following ponding, is shown in Fig. 3. In addition, the spectrum from a Kesterson Reservoir sediment is included in this figure. Note the consistent pattern of decreasing K-absorption edge position with decreasing valence.

Selenium K-Absorption Edge Spectra

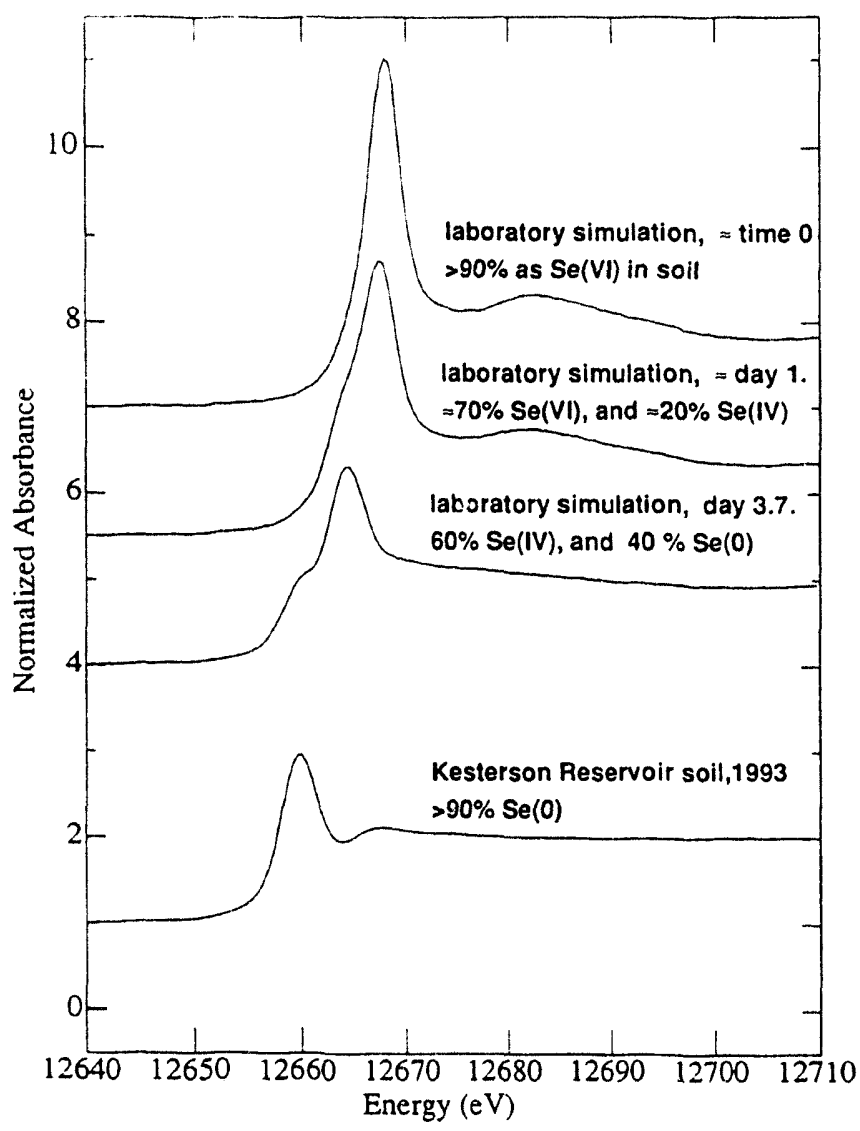

Fig. 3. X-ray absorption spectra of Se in sediments. The top three spectra are from laboratory simulations of $\mathrm{Se}$ reduction during ponding with $\mathrm{Se}(\mathrm{VI})$. These spectra provide the first direct measurements of the process of reduction from $\mathrm{Se}(\mathrm{VI})$ to $\mathrm{Se}(0)$. The bottom spectrum is from a Kesterson Reservoir soil. This spectrum provided the first direct evidence for the existence of $\mathrm{Se}(0)$ in Kesterson Reservoir sediments.
Despite certain limitations, these results provide examples of potential XAS applications in studies on cycling of Se and other trace elements and contaminants in the environment.

\section{Synchrotron X-Ray Microprobe Studies}

The synchrotron $x$-ray fluorescence microprobe at the National Synchrotron Light Source (NSLS beamline $\mathrm{X} 26 \mathrm{~A}$ ) in collaboration with Stephen $\mathrm{R}$. Sutton was used to map distributions of selenium and other elements within soil aggregates collected at Kesterson Reservoir, California, which were previously contaminated with seleniferous agricultural drain waters. Intact soil aggregates were scanned in 2 dimensions. Such analyses indicated large variations in Se concentrations occur across distances of $\approx 1 \mathrm{~mm}$ in individual soil aggregates.

The possibility that such heterogeneities were generated in anaerobic microsites within aggregates was investigated via laboratory simulations of Se contamination of individual aggregates. Twodimensional, synthetic aggregates of various sizes ( 8 to $25 \mathrm{~mm}$ nominal diameters) and water contents $(\approx 50$ to $100 \%$ saturation) were constructed from previously uncontaminated soils collected from near Kesterson Reservoir. Platinum electrodes were embedded at various points within some aggregates to provide indications of gradients in redox potentials. In some cases, sections of Scirpus roots were embedded within aggregates to provide local regions for enhanced microbial activity. Only the samples with embedded root sections displayed substantial heterogeneities in Se concentrations. An example of the generated heterogeneity is shown in Fig. 4. As much as 40-fold increases in total Se were observed in soils within $1 \mathrm{~mm}$ of such organic matter inclusions.

Such heterogeneities probably result from Se reduction to less mobile $\mathrm{Se}(\mathrm{IV})$ and insoluble $\mathrm{Se}(0)$ in the region of active organic matter decomposition, which in turn induces $\mathrm{Se}(\mathrm{VI})$ diffusion towards such zones for further reductive concentration. The measured Se accumulation around the embedded root section shown in Fig. 4 compared reasonably well with calculations based upon a simple transient $\mathrm{Se}(\mathrm{VI})$ diffusion model. Future studies of similar systems using a combination of SXRFM and valence-sensitive $x$-ray absorption spectroscopy will help test this hypothesis. 

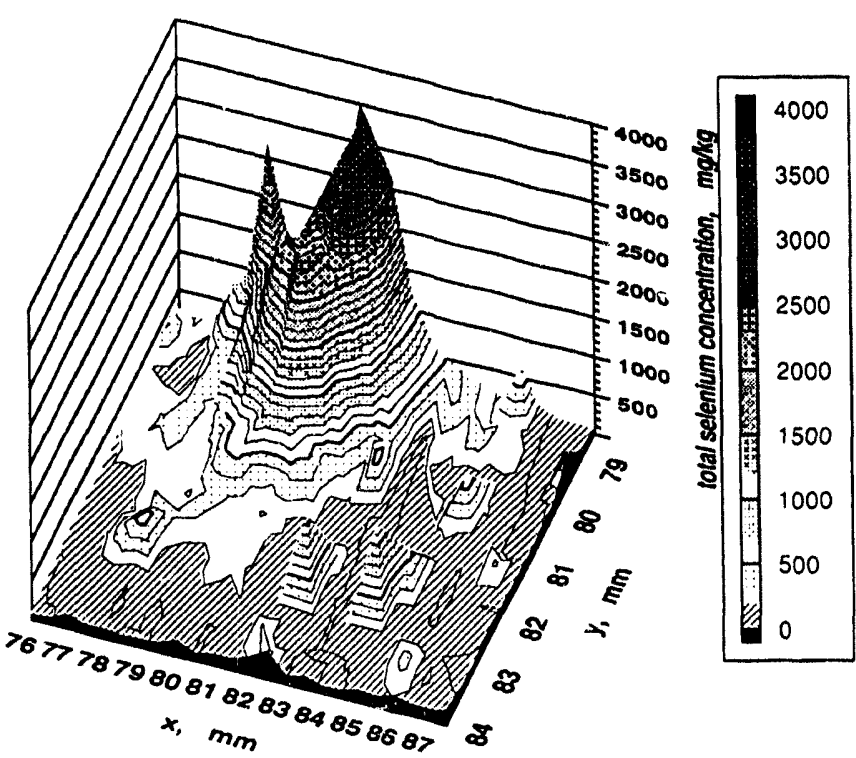

Fig 4. Synchrotron $x$-ray fluorescence microprobe map of Se accumulation around a laboratory-prepared decomposing Scirpus root embedded in a water-saturated sediment. Selenium was initially uniformly distributed as Se(VI) at $100 \mathrm{mg} / \mathrm{kg}$. The Se peak is centered around a $4-\mathrm{mm}$ diameter root cross section. Reduction of $S e(V I)$ to less soluble Se(IV) and insoluble Se(O) in the root zone probably induced diffusion of Se(VI) towards this region. Such small-scale heterogeneities in total Se were also observed in Kesterson Reservoir soils.
These SXRFM studies of Se in soils provide examples of the considerable research possibilities associated with this tool. Some near-future studies to be conducted at the ALS microprobe beamline will be designed to take advantage of the exceptional spatial resolution (approaching $1 \mu \mathrm{m}$ ) offered at this new facility. Summaries of these results were presented at the American Geophysical Union fall meeting in San Francisco.

\section{Publications}

I.J. Pickering, G.E. Brown, Jr., and T. K. Tokunaga, "XRay Absorption Spectroscopy of SeleniumContaminated Soils," to be submitted to Science.

T.K. Tokunaga, I.J. Pickering, and G.E. Brown, Jr., "Selenium Transformations in Surface Waters and Sediments during Transient Reduction," to be submitted to Soil Sci. Soc. Am. J.

T.K. Tokunaga, S.R. Sutton, and S. Bajt, "Heterogeneous Selenium Distributions within Individual Soil Aggregates: Evidence for Anaerobic Microsites Obtained With Synchrotron X-Ray Fluorescence Microprobe Mapping," to be submitted to Soil Science. 


\section{Energy and Environment Division}

\section{Exploratory RED Flow Pump Probe $X$-Ray Absorption and Emission}

Principal Investigators: Stephen P. Cramer, Jan van Elp, Judith L. Moore, Clayton R. Randall, and Xin Wang

Funding: $\$ 184,000$ (FY 93) $\$ 251,300$ (FY 92)

Project No. 92008

\section{Project Description}

Our group has been developing new types of $x$-ray spectroscopic techniques for characterizing metal sites in metalloproteins. There are three different techniques under development: (1) site-specific $x$-ray absorption (2) soft $x$-ray magnetic circular dichroism (MCD), and (3) flow-pump-probe spectroscopy. Sitespecific $x$-ray absorption spectroscopy uses small shifts in emission energies to obtain oxidation state or spin-state specific- $x$-ray absorption spectra. Soft $x$-ray $M C D$ has unique capabilities for investigating the magnetic and electronic structure of 3D transition metals in metalloproteins. The ultimate goal of flowpump-probe spectroscopy is to provide $x$-ray spectra on systems on the microsecond (or shorter) time scale.

\section{Accomplishments}

Site-specific $x$-ray absorption spectroscopy has been applied to a number of manganese-containing model complexes. The ultimate goal for this technique is to obtain specific EXAFS (extended $x$-ray absorption fine structure spectroscopy) spectra for different oxidation states or spin states in metalloproteins. We used a spherically bent $\mathrm{Si}(440)$ spectrograph to record highresolution x-ray fluorescence spectra of a physical mixture of two mono-valent $\mathrm{Mn}$ compounds of different oxidation states, $\mathrm{MnF}_{2}$ and $\mathrm{MnO}_{2}$. Figure 1 shows that site-selective XANES spectra taken by separately monitoring the $\mathrm{Mn}^{2+}$ and $\mathrm{Mn}^{4+}$ fluorescence emission of a physical mixture of $\mathrm{MnF}_{2}$ and $\mathrm{MnO}_{2}$ compare well with spectra taken of the pure compounds.

Soft x-ray absorption L-edge and MCD spectroscopy has been obtained on small proteins containing $\mathrm{Fe}$,

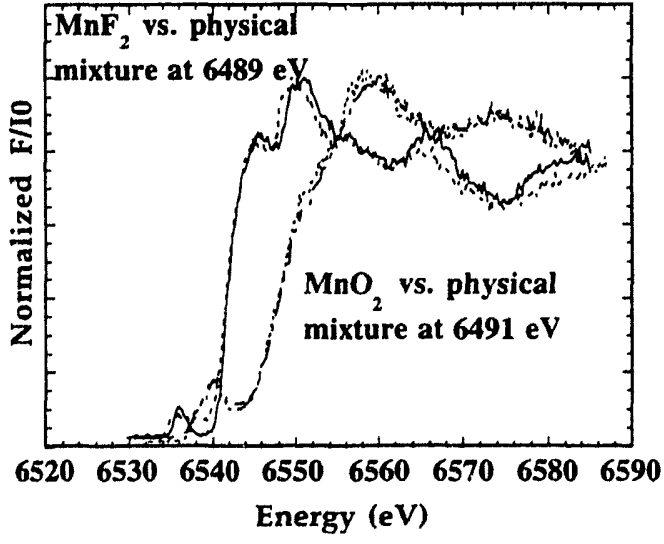

Fig. 1. Site-selective spectra of Mn model complexes.

$\mathrm{Co}, \mathrm{Ni}$, and $\mathrm{Cu}$. We have found that MCD effects are often strong $(\sim 30 \%)$, yet the measurements are extremely sensitive to experimental factors such as sample temperature, beamline stability, and photoreduction artifacts.

L-edge spectra are also interesting even without magnetic field effects. For example, we have studied the effects of electronic structure and symmetry on $\mathrm{Ni}$ $L$ edges by measuring the $L$ edges of several nickel compounds with different structural symmetries. Using ligand field atomic multiplet calculations, we find that there is a close relationship between the $\mathrm{Ni}$ L-edge spectral features and the electronic structure at the nickel site.

\section{Publications}

S. Mitra Kirtley, O.C. Mullins, J. van Elp, and S.P. Cramer, "Nitrogen Chemical Structures in Petroleum Asphaltenes and Coal by $X$-Ray Absorption Spectroscopy," Fuel 72, 133 (1993).

S. Mitra Kirtley, O.C. Mullins, J. van Elp, S.J. George, J. Chen, and S.P. Cramer, "Determination of the Nitrogen Chemical Structures in Petroleum Asphaltenes Using XANES Spectroscopy," J. Am. Chem. Soc. 115, 252 (1993). 
J. van Elp, S.J. George, J. Chen, G. Peng, C.T. Chen, L.H. Tjeng, G. Meigs, H.J. Lin, Z.H. Zhou, M.W.W. Adams, B.G. Searle, and S.P. Cramer, "Soft X-ray Magnetic Circular Dichroism-A Probe for Studying Paramagnetic Bioinorganic Systems," Proc. Natl. Acad. Sci. 90, 9664 (1993).

J. Chen, J. Christiansen, S.J. George, J. van Elp, R. Tittsworth, B.J. Hales, S. Al-Ahmad, D. Coucouvanis, N. Campobasso, J.T. Bolin, and S.P. Cramer, "Extended X-Ray Absorption Fine Structure and LEdge Spectroscopy of Nitrogenase Molybdenum-Iron Protein," Chapter 15 in Molybdenum Enzymes, Cofactors, and Model Systems, ACS Symposium Series 535, E. Stiefel, D. Coucouvanis, and W.E. Newton, eds., Washington, D.C. (1993).

S.J. George, M.D. Lowery, E. Solomon, and S.P. Cramer, "Copper L-Edge Spectral Studies: A Direct Experimental Probe of the Ground State Covalency in the Blue Copper Site in Plastocyanin," J. Am. Chem. Soc. 115, 252 (1993).

J. Chen, J. Christiansen, R.C. Tittsworth, B.J. Hales, S.J. George, D. Coucouvanis, and S.P. Cramer, "Iron EXAFS of $A$. vinelanidii Nitrogenase $\mathrm{Mo}-\mathrm{Fe}$ and $\mathrm{V}-\mathrm{Fe}$ Proteins," J. Am. Chem. Soc. 115, 5509 (1993).

S.J. George, J. van Elp, J. Chen, S. Mitra Kirtley, O.C. Mullins, and S. P. Cramer, "Soft X-Ray Absorption and X-Ray Magnetic Circular Dichroism in Biology," in Synchrotron Radiation in Biosciences, Oxford University Press, in press.

G. Pang, K. Hamalainen, F.M.F. de Groot, J.B. Hastings, D.P. Siddons, W.H. Armstrong, J. Moore, X. Wang, M. Gresh, O.C. Mullins, and S.P. Cramer, "Oxidation and Spin State Sensitivity and Selectivity from High Resolution Manganese X-Ray Fluorescence Spectroscopy," J. Am. Chem. Soc., in press.

J. Chen, R. Tittsworth, B.J. Hales, J. Christiansen, J. Bolin, N. Campobasso, and S.P. Cramer, "Refinement of a Model for the Nitrogenase Mo-Fe Cluster Using Single Crystal Mo and Fe EXAFS," Ang. Chem. 32, 1592 (1993).

J. van Elp, G. Peng, B.G. Searle, S. Mitra-Kirtley, Y.-H. Huang, M.K. Johnson, Z.H. Zhou, M.W.W. Adams, M.J. Maroney, and S.P. Cramer, "Electronic Structure and Symmetry in Nickel L-edge X-Ray Absorption Spectroscopy," J. Am. Chem. Soc., in press.

O.C. Mullins, S. Mitra Kirtley, J. van Elp, and S.P. Cramer, "Molecular Structure of Nitrogen in Coal from XANES Spectroscopy," Appl. Spectros. 47, 1268 (1993).
G.N. George, S.P. Cramer, T.G. Frey, and R.C. Prince, "X-Ray Absorption Spectroscopy of Oriented Cytochrome Oxidase," Biochem. Biophys. Acta, 1142, 240 (1993).

\section{Simulation of an Advanced Utility- Customer Interface}

Principal Investigators: Donald F. Grether, Hashem Akbari, Arthur H. Rosenfeld, Pravin P. Varaiya, Felix F. Wu, and Carl Blumstein

Funding: $\$ 75,000$ (FY 93) $\$ 75,000$ (FY 92)

Project No. 92009

\section{Project Description}

The electric utility industry is undergoing many changes. There is increasing demand for the services that electricity can provide and, at the same time, increasing economic, environmental, and regulatory constraints on the utilities' traditional approaches to meeting those demands by constructing new central station power plants, transmission lines, and distribution systems. Competition, small-scale generating units (e.g., gas fired turbines and renewable energy sources), independent power producers and cogenerators, demand-side management (DSM), programs, power wheeling, and energy storage are some of the factors contributing to a considerably more complex utility operating environment.

Key to the successful management of such systems is real-time monitoring and control through the use of power electronics, communication networks, and computers. This project focuses on one aspect of the larger problem, building upon the UC Berkeley background in $3 \mathrm{C}$ (communication, computation, and control) systems for utility automation and the LBL expertise in energy management and control systems (EMCS) for buildings. A resulting Utility-Customer $3 C$ (UC3C) system would yield energy efficiency improvements, real-time pricing, load leveling, and energy end-use monitoring, among other advantages.

\section{Accomplishments}

First, the present status and future trends in $3 C$ technologies were analyzed with a view toward utility- 
customer applications. Second, functional specifications and research needs for an integrated utilitycustomer energy management and control system that fully utilize the potential of $3 \mathrm{C}$ technologies were examined. Finally, work continued on a flexible software simulation environment intended to be suitable for the study and resolution of complex issues involved in the design of UC3C systems.

We reviewed recent progress and future trends in $3 C$ technologies including communication media and networking, computer hardware architecture and software engineering, and control algorithms. Several conclusions were reached. The so-called "digital convergence" is unifying all forms of communications-voice, data, image, video, etc. Optical fiber links carry millions of bits per second (Mbps) and broadband radio $(\mathrm{GHz})$ is emerging. SONET (synchronous optical network) transmission suitable for broadband integrated service digital network (BISDN) with channel capacity of $52 \mathrm{Mbps}$ and higher, and a multiplexing and switching technique called ATM (asynchronous transfer mode) have been developed for efficient multi-media transmission.

With computing power of workstations and capacity of communication networks increasing dramatically, dividing specific computing functions among multiple processors or processes in a networked workstations environment has become the mainstream of computing architecture. The distributed computing technology has led to a heterogeneous "enterprise computing" environment where mainframes, minis, personal computers, and workstations are all linked together. Software technology is undergoing a fundamental change to adapt to the rapid changing environment. The object-oriented approach supports modular design with a high degree of reusability, making it possible to design and maintain software made of replaceable components. The encapsulation of relevant data and procedures into self-contained objects makes it especially suitable for the distributed processing environment.

The economics of future UC3C systems favor distributed computing through broadband communication networking. After an initial investment in building the infrastructure, additional costs for bandwidth and computing power are relatively insignificant provided the system is so designed.

Within this overall context, a UC3C system is envisioned as integrating control functions in automated utility operation with energy control functions in buildings. EMCSs and microprocessor-based utility devices such as capacitor controllers, switches, etc., along with powerful database machines, and other processors, will engage in cooperative processing, which is embedded in the enterprise computing environment. The software will be object-oriented and consists of three conceptual components: a shared database, the application software, and a multi-media user interface.

Such integrated UC3C systems are complex. We have examined the multitude of functions and services they have to support and the heterogeneity of the devices they have to connect. The system must be designed to be modular, flexible, and extensible, with a high degree of reliability. The first necessary task is to construct a conceptual model of the system. We propose a model patterned after the network management systems of communication networks, where a framework to specify element (objects) in the system, called Structure of Management Information (SMI), is defined and a Management Information Base (MIB) is used for a unified representation of objects.

The design of the UC3C system requires analysis, optimization, and simulation of every aspect of the system. An integrated simulation environment for UC3C system is needed. The design of the simulator parallels the design of the system itself, therefore, the lesson learned will be extremely valuable. In particular, the design of the Information Base for the simulator is identical to that for the real system itself. In this project, we initiated the construction of a simple simulation of a UC $3 C$ system, which consists of a utility substation, a single feeder with one EMCS customer and many other noncontrolled customers. The objectives of the research are:

- to apply the object-oriented approach in the design of the UC3C Information Base

- to test the suitability of the object-oriented modular simulation package Ptolemy

- to demonstrate the feasibility of distributed computing in UC 3 C systems

- to develop methodology to study potential energy and cost savings of UC $3 \mathrm{C}$ system

The simple system has been implemented using the constructs (called stars) of Ptolemy, a software package developed by the Digital Signal Processing group at UC Berkeley. The first three objectives have been successfully achieved. The last one is near completion. 


\section{Inverse Problem to Direct Modeling of Combustion in Engines}

\author{
Principal Investigator: Antoni K. Oppenheim
}

Funding: $\$ 15,100$

Project No. 93007

\section{Project Description}

The aim of the project is to establish a close link between science and engine development and design by the provision of a numerical solution for the inverse problem with respect to the conventional direct modeling of combustion in engines. The computational program consists of three consecutive steps: (A) Thermodynamics, (B) Thermochemistry, and (C) Aerodynamics. From the first, one gets immediate information on the specific fuel consumption; from the second, an estimate is obtained on the formation of chemo-kinetically controlled pollutants, NOx and $\mathrm{CO}$; and from the third, guidelines are provided for the fluid mechanical control of the exothermic process of combustion, pertaining in particular to the reduction of unburned hydrocarbon emissions.

\section{Accomplishments}

The study yielded manuscripts for two papers: (1) "Can the Maximization of Fuel Economy be Compatible with the Minimization of Pollutant Emissions," and (2) "A Thermochemical Phase Space for Combustion in Engines," as well as (3) a project proposal on "Design Analysis for Optimal Execution of the Exothermic Process of Combustion in Natural Gas Fueled Engines," to be financed by contract with Southwest Research Institute.

The first paper provided rational arguments why the mandate of the President's Clean Car Initiative to, at least, double the mileage per gallon of fuel and simultaneously eliminate pollutant emissions that should be met by radical improvements in the execution of the exothermic process of combustion in engines-a task involving the transformation of the contemporary mode of FTC (Flame Traversing the Charge) to microprocessor controlled FMC (Fireball Mode of Combustion), the epitome of DISC (Direct Injection Stratified Charge) engine, featuring late injection and stratified combustion using a PCJ (Pulsed Combustion Jet) system. It introduces also a systematic procedure for engine development, consisting of thermodynamic and thermochemical computations, that, with an aerodynamic analysis of the turbulent field, lays the groundwork for engine design by a systematic procedure of IDT (Indicator Diagram Tailoring).

The second paper introduced the concept of a thermochemical phase space, based on the recognition that the combustion system is nonlinear, and utilizing, therefore, the classical concept of nonlinear mechanics: a space whose coordinates are the dependent variables of the problem. In the case at hand, they consist of all the thermochemical parameters of the system. The dimension of this space is thus equal to the number of degrees of freedom. We name it the Le Chatelier space. Its major asset lies in providing information, expressed in terms of trajectories or manifolds, on the global effects of the thermochemical processes that take place in the physical space of the combustion chamber. Obtained thereby is an analytical insight into the effective mechanism of the exothermic process of combustion. Application of the method is illustrated by the evaluation of advantages accruable for a premixed charge engine. It is shown, in particular, that if, instead of a throttled homogeneous combustion, the exothermic process is executed in a FMC version of a PCJ generated DISC system, the engine can be rendered the ability for part-load operation at wide open throttle, with significant gains in fuel economy and concomitant reduction in pollutant emissions. Such a mode of combustion occurs within large-scale vortex structures generated and sustained by pulsed jets.

The proposal offered to provide assistance in assessing and optimizing the performance of natural gas fueled engines. Its particular concern is with the execution of the exothermic process of combustionthe crucial event associated with the production of motive force, consumption of fuel and concomitant generation of pollutants, that so far has been vastly overlooked in engine development and design.

The project provided the background for the Conference on Scientific Countermeasures for Pollutant Emissions from Internal Combustion Engines held on November 12 at UCB under the auspices of the Institute of Transportation Studies. Among the more than 40 attendees of the conference were staffs from the California Energy Commission (CEC) and the California Air Resources Board (CARB), industrial representatives from Rockwell, PG\&E, Southern California Gas Company, BKM and Southwest Research Institute, as well as scientists from LBL, LLNL, UC at Davis, Irvine, San Diego, and Berkeley. Major recommendations of the conference were as follows: 
(1) Let us take the bull by the horns and do our best, ahead of the rest of the country, in responding to the Clinton/Gore mandate of the Clean Car Initiative.

(2) Take advantage of California's aerospace industry to provide assistance to the Big 3 in the development of a Clean Engine that will meet the CARB Zero Emission Vehicle standards taking into account, of course, that the electrical energy for motors, that today are expected to provide the ultimate solution, is derived from fossil fuels.

\section{Publications}

A.K. Oppenheim, "Can the Maximization of fuel Economy be Compatible with the Minimization of Pollutant Emissions," to be presented at the Society of Automotive Engineering Congress, Detioit, MI, February 28-March 4, 1994.

A.K. Oppenheim, "A Thermochemical Phase Space for Comhustion in Engines," to be presented at the International Symposium on Combustion, Irvine, CA, July 31-August 5, 1994.

\section{New Approaches to Assessing the Oceans as Carbon Dioxide Sinks}

Principal Investigators: Ronald L. Ritschard, Mary Quinby-Hunt, Kenneth H. Orvis, and Patrick Neale

Funding: $\$ 104,300$ (FY 93) $\$ 129,300$ (FY 92)

Project No. 92010

\section{Project Description}

The world's oceans cover approximately $70 \%$ of the surface of the earth. The ocean provides sources and sinks for $\mathrm{CO}_{2}, \mathrm{CH}_{4}$, and other gases important to the global carbon budget. The importance of oceanic biogeochemical processes in the global carbon budget is clear, but many uncertainties remain. Not all $\mathrm{CO}_{2}$ that is released into the atmosphere is accounted for by uptake into known terrestrial and oceanic sinks. A better understanding of the ocean's carbon budget is essential for understanding the mechanisms and kinetics of climate control.
Oceanic phytoplankton are an important component of the global carbon budget. Phytoplankton consume $\mathrm{CO}_{2}$ and may release it to the water column when they decompose. They usually contain chlorophyll $a$, a substance readily detected by satellites. However, typical conversion of remote-sensing data to phytoplankton biomass is often inaccurate and requires extensive correction; computer programs developed to date are inadequate, and algorithms developed for one part of the world often result in inaccurate estimates for other parts of the world, particularly where riverine inputs are a complicating factor. Therefore, the possibility that phytoplanktonic fluorescence might be used for estimating oceanic phytoplanktonic processes should be explored.

Methane formed from the decomposition of phytoplankton is another important, but less explored, carbon sink. After formation, methane may be sequestered in hydrates (clathrates). Clathrate hydrates are solids in which water forms a rigid lattice of cages that may contain a guest molecule. In the marine environment, the guest molecule is often methane, carbon dioxide, or hydrogen sulfide (all formed during phytoplanktonic decomposition). The existence of large regions of methane clathrates on the ocean floor has been suggested based on interpretation of acoustic records: estimates report that on the order of $\sim 10^{19} \mathrm{~g}$ of methane exist in hydrates globally.

Clathrates are also of interest in two additional areas: as a potential fossil fuel resource and as a submarine geohazard. If the estimate of the resource is accurate, then the amount of organic carbon that may be stored in gas hydrates is comparable to the total quantity of organic carbon stored in other global resources. Even if the estimate is off by a factor of two, this is a vast, untapped resource. Because the methane in gas hydrates and some of the hydrates themselves are formed in marine sediments, consolidation and cementation of the sediments can be inhibited. The resulting structure may therefore be weaker and more prone to slumps, debris flows, or slides in the event of a destabilizing occurrence, such as earthquakes, pressure changes due to changing sea level, or perturbation during drilling or oil/gas production activity. Clathrate-related slumps, debris flows, and slides have been reported and could present hazards to human activities, not only to oil/gas drilling and production, but to communications and other cables on affected slopes.

During the second year of this project, the potential for using the fluorescence of phytoplankton as a means of estimating phytoplankton productivity 
continued to be explored. A study of the conditions of methane clathrate formation under marine conditions was initiated at LBL. Once the ability to investigate clathrate formation is established, the results can be applied to a study of their importance to the global carbon budget and climate regulation. The preliminary results of this study are presented below.

\section{Accomplishments}

\section{Phytoplankton Fluorescence}

In attempting to improve the measurement of oceanic phytoplankton by remote sensing, a new approach via monitoring of fluorescence of chlorophyll $a$ in surface phytoplankton was explored. We were able to demonstrate that this technique may be used as a probe to study phytoplanktonic processes, as fluorescence from chlorophyll $a$ - containing phytoplankton is strongly dependent on light level. As a monitor of oceanic productivity this technique is limited by the dependence of chlorophyll $a$ fluorescence on light level, thus necessitating an extremely complex correction to any estimate of productivity. Further, much of the oceanic biomass resides at depths not detected by remote sensors; also, a significant portion of marine biomass does not contain chlorophyll $a$.

\section{Marine Gas Clathrates}

Both methane and carbon dioxide clathrates have now been prepared at LBL. Preliminary experiments were conducted using deaerated distilled water and commercial methane and carbon dioxide. A windowed autoclave permitted observation of clathrate formation at high pressure. $\mathrm{CO}_{2}$ clathrates were found to form readily at 900 psi and from 5$8^{\circ} \mathrm{C}$. The clathrates were brought to room temperature and pressure and removed from the autoclave to permit examination. They were found to be polycrystalline, white (similar in appearance to dry ice) and could be described as effervescent ice. Methane clathrates formed at higher pressures ( 1200 psi) and at similar temperatures. In order to observe them at room temperature and pressure, it was necessary to rapidly decompress. The material retrieved was multicrystalline and the vapor released as the clathrate evaporated could be ignited.

The chemistry and thermodynamics of formation of methane in anoxic sediments has been examined to better understand the environment under which they form. The thermodynamics and kinetics of marine gas hydrates have generally been studied in distilled water in the presence of oxygen. Now that it has proven possible to form clathrates at LBL under dis- tilled water/oxic conditions, studies are continuing under more natural conditions using water with appropriate concentrations of components found in anoxic sea water. This study will lead to a better understanding of the stability of gas hydrates and permit a more detailed exploration of their contribution to climate regulation, their resource potential, and problems they may present as geohazards.

\section{Publications}

P.J. Neale and R.L. Ritschard, "Study of Marine Phytoplankton Fluorescence-Photosynthesis Relationship," in preparation.

K.H. Orvis and R.L. Ritschard, "Reanalysis of CZCS Images Acquired under West Coast Continental and Urban Air Mass Regimes," in preparation.

\section{Materials Processing and Device Fabrication of Wide-Bandgap Materials}

Principal Investigator: Michael D. Rubin

Funding: \$90,000 (FY 93)

Project No. 92011 $\$ 75,000$ (FY 92)

\section{Project Description}

The general purpose of this project is to develop wide-band gap materials and devices for electronic and optoelectronic applications. Applications include blue or ultraviolet LEDs and laser diodes as well as high-power, high-frequency, or high-temperature electronics. Our specific short-term goals are to produce the best quality p-type $\mathrm{GaN}$ and fabricate an efficient blue LED. GaN is of special interest because it has a direct band gap ( $3.4 \mathrm{eV})$, and it is completely miscible and vacuum compatible with other III-V nitrides such as AIN and InN.

Our approach to developing devices based on wideband-gap semiconductors is to concentrate at first on the materials themselves. The strong chemical bonds in GaN have many advantages in terms of properties, but make defect-free bulk or epitaxial material difficult to obtain. Furthermore, because of the lowsolubility and high-equilibrium vapor pressure of 
nitrogen, GaN tends to form with high concentrations of nitrogen vacancies. Poor lattice and thermalexpansion match with practical substrates cause other types of defect generation. We study these defects and the film characteristics by a variety of technique such as temperature-dependent Hall measurements, cathodoluminescence, photoluminescence, magnetic resonance, $x$-ray diffraction, and electron microscopy. Our recently completed deposition system uses the principle of ion-assisted molecular-beam epitaxy (MBE) to produce extremely high-quality films. By starting with this good intrinsic material, we have been able to create p-type conduction in several different ways.

\section{Accomplishments}

In order to minimize ion-impact damage during the film growth, the substrate to anode potential should be as small as possible. The minimum anode potential of $+30 \mathrm{~V}$ combined with substrate biasing of $+18 \mathrm{~V}$ is expected to reduce the impinging $\mathrm{N}_{2}{ }^{+}$ion energy to about $10 \mathrm{~V}$. When this technique was used in combination with low temperature and high $\mathrm{N}_{2}{ }^{+}$ flux, $\mathrm{p}-\mathrm{GaN}$ was obtained without intentional doping. These undoped films have hole concentrations of $5 \times$ $10^{11} \mathrm{~cm}^{-3}$ and hole mobilities of over $400 \mathrm{~cm}^{2} / \mathrm{V} \mathrm{s}$ at $250 \mathrm{~K}$. From the slope of the carrier concentration versus temperature shown in Fig. 2(a), an activation energy of $0.29 \mathrm{eV}$ is obtained. The strong temperature dependence for the mobility, Fig. 2(b) indicates that phonon scattering dominates the mobility at room temperature. These electrical parameters compare quite well with values for $\mathrm{GaAs}$ when adjusted for the strength of the electron-phonon coupling and the Debye temperature, despite the higher level of impurities in our ion beam MBE system compared to conventional $\mathrm{GaAs} \mathrm{MBE}$ and the wider $\mathrm{x}$-ray peaks of the GaN films.

Diffusion of $\mathrm{Mg}$ into unintentionally doped n-type $\mathrm{GaN}$ films resulted in conversion to p-type material. The films were grown at $600^{\circ} \mathrm{C}$ without substrate bias. The diffusion was performed in a sealed ampoule at atmospheric $\mathrm{N}_{2}$ pressures and $850^{\circ} \mathrm{C}$ for 80 hours. Hall measurements at room temperature indicated a hole concentration of $2 \times 10^{16} / \mathrm{cm}^{3}$ and a mobility of $12 \mathrm{~cm}^{2} / \mathrm{Vs}$ at room temperature. Higher diffusion temperatures resulted in the removal of the film by decomposition and evaporation. Lower diffusion temperatures were not successful in converting the material to p-type.
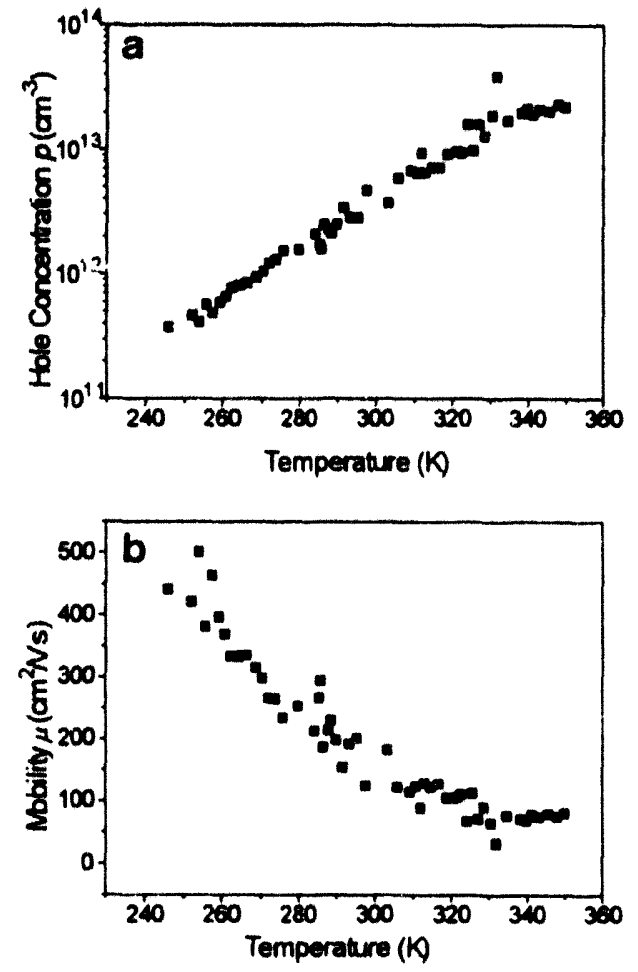

Fig. 2. (a) Hall carrier concentration and (b) Hall mobility versus measurement temperature for a biased undoped $p$ GaN film.

$\mathrm{N}$-type films grown at temperatures of $600^{\circ} \mathrm{C}$ without substrate bias were also implanted with large doses $\left(2.02 \times 10^{14} \mathrm{~cm}^{-2}\right)$ of $\mathrm{Mg}$ using a metal-vapor vacuum-arc ion source. High-energy (80-100 keV) $\mathrm{Mg}$ implants introduced strain and defects into the $\mathrm{GaN}$ films. Even after annealing at $800^{\circ} \mathrm{C}$ for 30 minutes in $\mathrm{N}_{2}$ the x-ray peaks did not return to their initial positions. A high concentration of mid-gap states were detected with optical absorption, presumably preventin the material from becoming p-type by the shallow Mg dopant. Films implanted with $\mathrm{Mg}$ ions at lower energies $(40-60 \mathrm{keV})$ recovered their original lattice parameter as determined by $x$-ray diffraction (see Fig. 3). Hot-point probe tests confirmed that these lower energy implants produced p-type conduction. This is the first report of p-type $\mathrm{GaN}$ using ion implantation. Room temperature resistivities of the implanted films are $4 \Omega-\mathrm{cm}$.

Finally, a Knudsen cell for direct in-situ coevaporation of $\mathrm{Mg}$ was used. At a $\mathrm{Mg}$ source temperature of $180^{\circ} \mathrm{C}$ and a substrate bias of $18 \mathrm{~V}$, the 


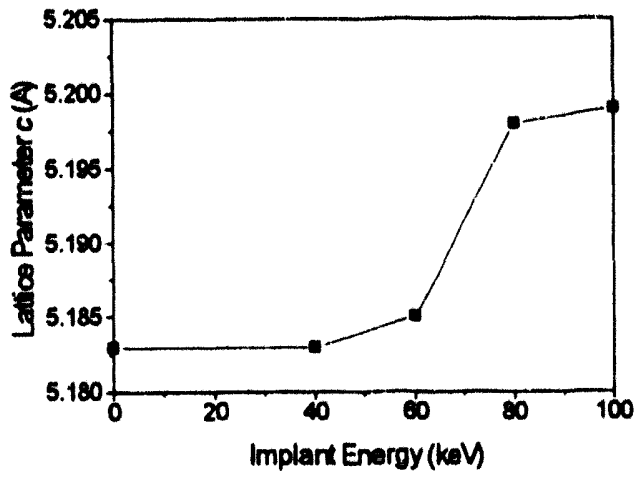

Fig. 3. Strained lattice parameters of films implanted with $\mathrm{Mg}$ at various energies.

films were found to exhibit hole conduction. Hall measurements indicated a hole concentration of $2 \times 10^{17} \mathrm{~cm}^{-3}$ and hole mobilities of $<1 \mathrm{~cm}^{2} / \mathrm{V} \mathrm{s}$ at room temperature.

In summary, p-type conduction was obtained in ion beam MBE films by a variety of doping methods in- cluding ion implantation, diffusion and coevaporation of $\mathrm{Mg}$. These results clearly indicate that the key to producing the control of hole-doping lies primarily in reducing the defects in the base material.

\section{Publications}

M. Rubin, N. Newman, J.S. Chan, T.C. Fu, and J.T. Ross, "P-Type Gallium Nitride by Reactive Ion-Beam MBE with Ion Implantation, Diffusion, or Coevaporation of $\mathrm{Mg}$," to be published in Appl. Phys. Lett.

J. Ross, M. Rubin, and T.K. Gustafson, "Single-Crystal Wurtzite GaN on (111) GaAs with AlN Buffer Layers Grown by Reactive Magnetron Sputtering," J. Mater. Res. 8, 2613 (1993).

N. Newman, J. Ross, and M. Rubin, "Thermodynamic Processes Involved in the Growth of Epitaxial GaN Thin Films," Appl. Phys. Lett. 62, 1242 (1993). 


\section{Engineering Division}

\section{Development of Microchemical Methods for Biological Assays}

Principal Investigators: Joseph M. Jaklevic and Jocelyn C. Schultz

Funding: $\$ 46,900$

Project No. 93008

\section{Project Description}

The project is to develop methods for performing biochemical procedures in submicroliter quantities arrayed in multiple reaction cells. The result of such a program will be the capability of performing biochemical assays in large numbers at reduced costs. The methods will have numerous applications in emerging biotechnological areas such as genome mapping and sequencing, and large-scale clinical assays.

There are three areas where miniaturization of laboratory procedures will be required and where proposed approaches appear feasible.

1. Large arrays of small-area reaction cells can be fabricated using standard photolithographic nanofabrication methods. Successive masking and processing steps can be used to create physical wells in an appropriate substrate. Alternatively, patterned surface treatments can be used to create hydrophobic barriers around individual reaction cells or to activate the surface attachment of reagents at spatially separated locations.

2. Dispensing nanoliter volumes can be achieved with dispensers based on drop-on-demand ink-jet printer technology. Arrays of these dispensers, each of which could be filled with microliter quantities of a different reagent, may be necessary. Preliminary measurements using pressurized reservoirs, small capillaries, and pulsed solenoid valves have demonstrated the principle of pulsed reagent dispensing of microliter volumes. Further studies are required to determine this method's lower limit of applicability.

3. There are a number of fluorescence assays that are commonly used to detect biological activity such as hybridization of complementary DNA in genome mapping. With high-resolution scanning hard- ware based on laser excitation through fiber optic bundles, small reagent quantities can be detected. This will also be investigated as a method for detecting biological activity at a sensitivity and spatial resolution commensurate with the array geometry.

The proposed program is to explore the capabilities of each of these technologies with emphasis on producing an integrated system for performing biochemistry at a level approaching one nanoliter volumes. At this level of resolution it would be possible to perform 10,000 reactions in a $1 \mathrm{~cm}^{2}$ area. Even a modest approach toward this limit represents a significant advance in current state of the art.

\section{Accomplishments}

A collaboration with ]. Gray (UC San Francisco and LBL) was pursued with a goal to produce arrays of DNA clones covalently bound to glass slides, as a way to automate Gray's technique of comparative genomic hybridization. As far as we have been able to determine, no one has reported binding DNA fragments in an array of $100-\mu \mathrm{m}$ sized dots, our target scale. As the first step in this project, we identified several methods that could be used to covalently bind samples of DNA to glass slides at spatially separated locations. One method, proposed to us by Prof. A. Glazer (UC Berkeley), would involve a photoactivated coupling reaction between a polymer, multiply substituted with a carbene-generating ligand and covalently bound to the glass slide, and the DNA fragments. The synthesis of the necessary reagents and the initial testing of the concept are being carried out by Glazer and co-workers. If promising, we will undertake the experiments necessary to optimize this system. It may be necessary to localize the reagents in reaction cells fabricated by creating hydrophobic barriers on the glass slide. The surface treatments necessary to pattern the slide have been identified.

An apparatus was assembled, and studies were begun to determine the lower limits achievable by metering a pressurized liquid with a pulsed solenoid valve. Using a fast-response solenoid valve and a short length of capillary tubing ( $0.020 \mathrm{in} . \mathrm{OD}, 0.010 \mathrm{in}$. ID) for a dispenser tip, volumes as small as 0.2 microliter could be dispensed, but good reproducibility (better than $10 \%$ ) was achieved only for volumes of 0.5 
microliter and above. Other designs of the dispenser tip, that would be expected to improve the reproducibility at the lower volumes and extend the lower limit somewhat, will be explored in the future.

To achieve nanoliter dispensing of biological fluids, we turned to ink-jet printer technology. We began developing a drop-on-demand dispenser using a piezo-ceramic transducer to generate the pressure wave that will result in a subnanoliter-sized droplet being expelled from the nozzle. The first dispenser was built from piezo-ceramics on hand, and a watch jewel with a $100-\mu \mathrm{m}$ hole as the nozzle. Using this test dispenser, progress was made in maximizing the effect of the piezo activation on the movement of the meniscus at the nozzle and in understanding the effect of different experimental variables. In the future, electronics that will allow us to apply the optimal voltage pulses to the piezo will be obtained. We also plan to develop an apparatus to provide strobed microscopic viewing of the nozzle so that the droplet formation process can be followed. Finally, to build an improved dispenser, custom-made piezoceramics will be ordered.

\section{Custom Gallium Arsenide Integrated Circuit Design for Applications in Nuclear and Particle Physics}

Principal Investigators: Stuart A. Kleinfelder, Eric Beuville, and Oren B. Milgrome

$$
\begin{aligned}
\text { Funding: } \$ 70,000 \text { (FY 93) } & \text { Project No. } 92012 \\
\$ 27,400 \text { (FY 92) } &
\end{aligned}
$$

\section{Project Description}

This investigation seeks to develop the capability to design custom microwave speed GaAs integrated circuits at LBL. The project initiated explorations into the techniques of GaAs design, necessary computeraided design tool integration, forging part fabrication pathways, and gaining experience with very highspeed part testing. A specific goal was to proceed to the point of actually fabricating and validating one or more significant experimental GaAs integrated circuits. The design projects implemented include basic parameter measurement circuits, analog building blocks including high-speed amplifiers and buffers, a complete photoreceiver circuit, digital subblocks including emitter-coupled logic-level receivers and transmitters, and two medium-scale digital circuits including a 32-bit adder and a programmable clock skew generator.

\section{Accomplishments}

The majority of the research was aimed at developing the ability to accurately model GaAs circuits. Such models are necessary during the design process, particularly of analog circuits. The models available elsewhere were useful only for digital circuits. Two chips containing different sizes of enhancement and depletion fets and resistors with back-gate contacts made with Schottky and ohmic contacts were designed and fabricated. These devices allowed comparison of simulation results and actual device behavior. Effects studied included DC characteristics, back- and side-gate effects, "looping" (hysteresis) effects, "kink" effects in drain current of small devices, "lag" effect (frequency dependence of drain and source access resistance), and noise performance. Serious flaws in simulation accuracy were identified, and means to improve the predictive quality of these simulations were investigated.

GaAs technology is the dominant vehicle for optical transmitter and receiver circuits. Our investigations included attempts to combine photodetectors and associated amplifiers. Four types of photodetectors were produced. The first is a photoconductor with an implanted active region. This is similar to a fet, but instead of a gate modulating current between the ohmic contacts, incident light modulates the current. In the second type, the active layer is omitted, leaving the ohmic contact with just the semi-insulating p-type $\mathrm{GaAs}$ between them, forming a $n+p-n+$ structure. The third is a metal-semiconductor-metal (MSM) photodiode in which the ohmic contacts are replaced by gate-metal contacts, corresponding to back-to-back Schottky diodes. The last is an asymmetric photodiode, which can operate without bias. This is identical to the $n-p-n$ type except that one of the junctions is replaced with gate metal, resulting in a metal-p-n+ (M-p-n) structure. It has different built-in potentials at the two junctions, so that under illumination the photogenerated voltages differ and do not cancel. A transimpedance amplifier was integrated with each of the photodetectors. Measurements of the dark current and photocurrent for each of the photodetectors were successfully performed. 


\section{Extended X-Ray Absorption Fine Structure Spectroscopy (EXAFS) in the 1-4 keV Region}

Principal Investigators: Carolyn S. Rossington, Kim M. Yu, Wladyslaw Walukiewicz, and Joseph M. Jaklevic

Funding: $\$ 60,900$ (FY 93) $\$ 75,500$ (FY 92)

Project No. 92014

\section{Project Description}

This project continued the development of methods for performing fluorescence extended $x$-ray absorption fine structure spectroscopy (EXAFS) in the 1-4 $\mathrm{keV}$ region. There are a number of low $\mathrm{Z}$ elements of significant material and biological interest with absorption edges in this region, but which have not been easily accessible for EXAFS study due to the experimental difficulties associated with low-energy $x$-ray attenuation and detection. A new $x$-ray spectrometer was designed and built at LBL during the first year of this project. specifically for lowenergy EXAFS work, and vras to be optimized further during the second year. The semiconductor materials systems of $\mathrm{CdTe}: \mathrm{Cl}$ and $\mathrm{ZnSe}: \mathrm{Cl}$ were chosen for initial study due to their importance in II-VI semiconductor research and to demonstrate the feasibility of the low-energy EXAFS technique. Additional materials of CdTe nanocrystals, CdMnTe thin films, and heavily doped (via ion implantation) InP:Ge, $\mathrm{ZnSe}: \mathrm{Fe}, \mathrm{ZnTe}: \mathrm{Fe}$, and $\mathrm{GaAs}: \mathrm{Mn}$ were also to be studied. The EXAFS measurements were to be performed at the Stanford Synchrotron Radiation Laboratory (SSRL), and the resulting technology and expertise are intended to open an area of research applicable to the $x$-ray energy range provided by bending magnet beamlines at LBL's Advanced Light Source synchrotron.

\section{Accomplishments}

The materials studied during the past year included $\mathrm{Cl}$-implanted $\mathrm{CdTe}$ and $\mathrm{ZnSe}, \mathrm{Fe}$-implanted $\mathrm{ZnSe}$ and $\mathrm{ZnTe}$, Ge-implanted InP, Zn-diffused GaAs and InP, and CdTe nanocrystals. In addition to the EXAFS measurements, complementary materials characterization was done using Hall effect measurements, secondary ion mass spectroscopy, Raman spectroscopy, and proton-induced $x$-ray emission. The $x-$ ray spectrometer for the EXAFS work was designed and built at LBL for high-rate, high-energy resolution $x$-ray fluorescence (XRF) spectroscopy. The first stage of the spectrometer was completed during the first year of the project and was capable of collecting XRF data for synchrotron EXAFS studies $>5 \mathrm{keV}$. Further optimization of the spectrometer during the second year resulted in improved energy resolution and count rates necessary for $<5 \mathrm{keV}$ EXAFS studies.

\section{EXAFS Results}

Although several materials systems have been studied, only two will be detailed here. The first example demonstrates the characteristics of the x-ray spectrometer that was developed for the low-energy EXAFS work. Figure 1 shows the raw XRF spectrum from Cl-doped $\mathrm{ZnSe}$, fluoresced with a $2.8 \mathrm{keV}$ synchrotron beam. The $2622 \mathrm{eV} \mathrm{Cl} \mathrm{K}$-alpha peak is well-separated from the $2830 \mathrm{eV}$ monochromator scatter peak, due to the excellent energy resolution of the spectrometer. The spectrometer parameters were: $150 \mathrm{eV}$ full-width at half-maximum (FWHM) resolution at $2.6 \mathrm{keV}, 2.5 \mu \mathrm{s}$ peaking time, and 45,000 counts per second throughput rate. This represents a 2.5 -fold increase in count rate at equivalent energy resolution over conventional spectrometers. In addition, the peak-to-background is approximately three times better, due to the geometry of the new $x$-ray detector, compared with existing commercial systems. The raw XRF data shown in Fig. 1 were used for subsequent EXAFS analysis.

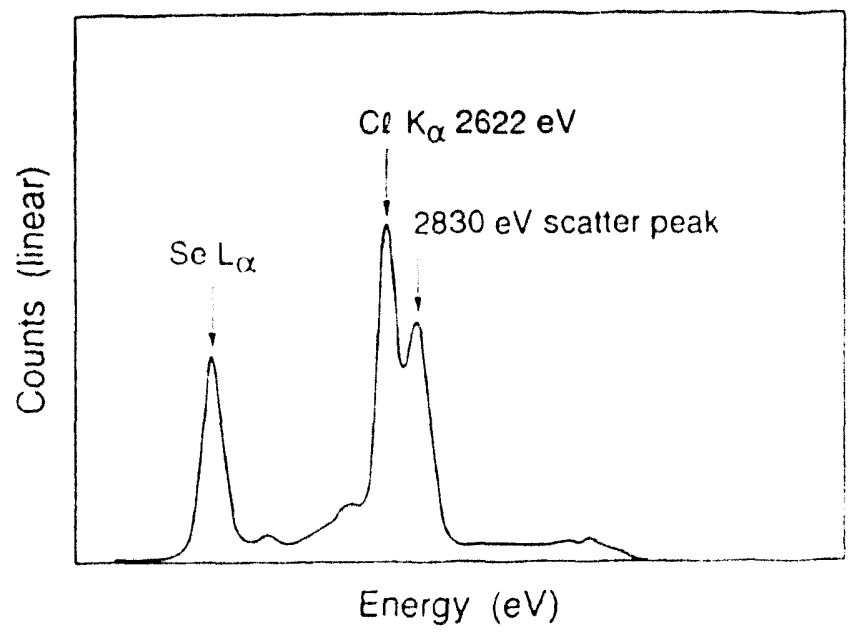

Fig. 1. X-ray fluorescence spectrum of $\mathrm{Cl}$-doped $\mathrm{ZnSe}$ fluoresced by a $2830 \mathrm{eV}$ monochromator synchrotron source, used for subsequent EXAFS analysis. Spectrometer parameters: $2.5 \mu$ s peaking time, 45,000 counts per second throughput rate, $\sim 150 \mathrm{eV}$ FWHM resolution at $2.6 \mathrm{keV}$. 
The second example is the EXAFS analysis of heavily Ge-implanted InP. InP material was Ge-implanted at both liquid nitrogen temperature (LNT) and room temperature (RT) and then annealed at $880^{\circ} \mathrm{C}$ for 10 seconds. Figure 2 shows the Fourier transform magnitudes as a function of radial distance from the

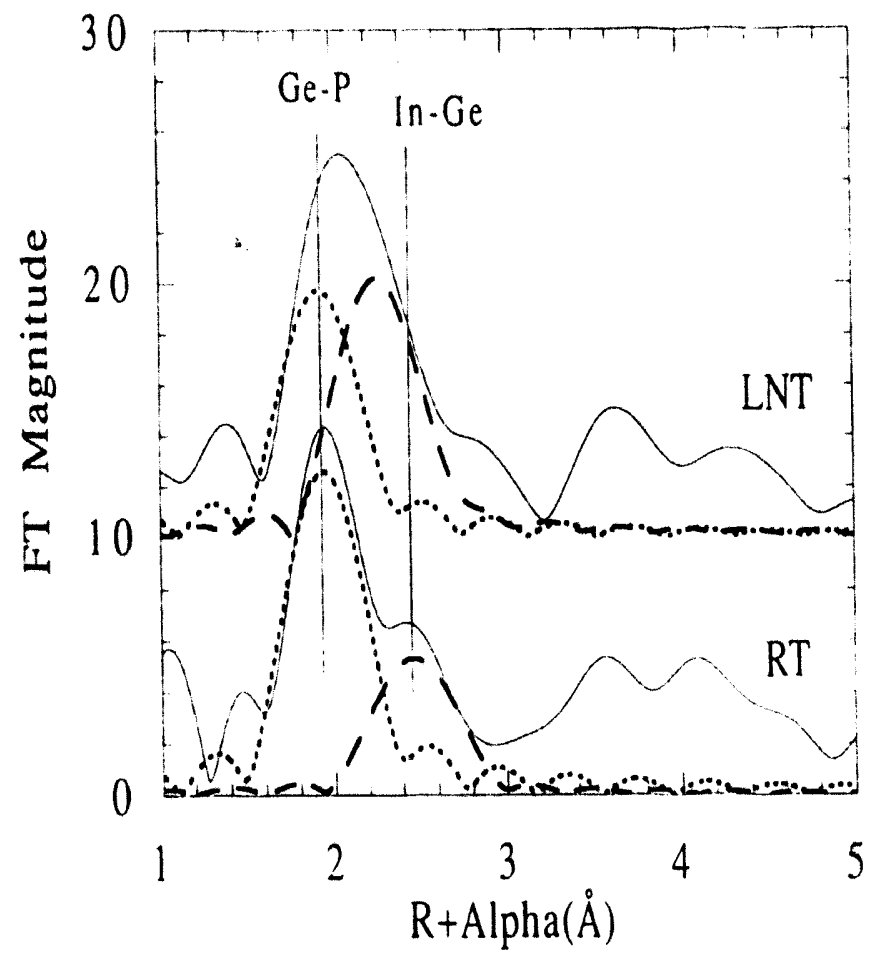

Fig. 2. Fourier transform magnitudes of the EXAFS oscillations from the LNT and RT Ge-implanted InP. The dashed curves represent the Fourier transform of the best-fit data.
Ge ( $R+$ Alpha, in A, uncorrected for phase shift), for the two different implantation conditions. These data clearly show the amphoteric nature of $\mathrm{Ge}$ in $\operatorname{InP}$ : Ge sits on both In and P sites and is electrically active on both sites, thus being both a donor and acceptor. The data also show a difference in the local environment for the two Ge sites in the LNT- and RT-implanted samples. The ratio of Ge on In sites, to Ge on P sites, is consistent with the electrical behavior of the material. The n-type activation efficiency in the RT-implanted samples was approximately a factor of two higher than in the LNT-implanted samples.

Although the amphoteric nature of Ge in InP was previously predicted based on theoretical calculations, this is the first time direct evidence has shown this amphoteric substitutionality.

\section{Publications}

K.M. Yu, J.W. Ager III, N. Derhacobian, R. Giauque, J.M. Jaklevic, C.S. Rossington, W. Walukiewicz, M. Wesela, and P. Becla, "EXAFS Analysis of Magnetic Semiconductor Thin Films Synthesized by Ion Beam Technique," Mater. Res. Soc. 307, 179 (1993).

K.M. Yu, W. Walukiewicz, L.Y. Chan, E.E. Haller, C.S. Rossington, N. Derhacobian, and J.M. Jaklevic, "Local Environment of $\mathrm{Zn}$ in Highly P-Type $\mathrm{Zn}$-Diffused GaAs," in preparation.

K.M. Yu, A.J. Moll, W. Walukiewicz, N. Derhacobian, and C.S. Rossington, "Amphoteric Substitutionality and Lattice Distortion of Ge in InP," submitted to Appl. Phys. Lett. 


\section{Environment, Health and Safety Division}

\section{Modification of an Andersson- Braun-Type Remmeter to Extend Its Sensitivity to $400 \mathrm{MeV}$}

Principal Investigator: Rai-Ko S. Sun

Funding: $\begin{aligned} & \$ 46,500 \text { (FY 93) } \\ & \$ 98,900 \text { (FY 92) }\end{aligned}$

Project No. 92015

\section{Project Description}

A remmeter is an instrument that measures the absorbed dose in tissues (dose equivalent) from a radiation field. The original purpose of this project was to modify the Andersson-Braun-type remmeter, in both construction and material, to extend its responses to medium- and high-energy neutrons $(>10 \mathrm{MeV})$. A simulation of the modification, using the Monte Carlo codes LAHET and MCNP, was very successful. By introducing a lead sleeve surrounding the $\mathrm{BF}_{3}$ detector, the remmeter response at high energies has been extended to the $\mathrm{GeV}$ range. The theoretical calculations can be verified experimentally. The final goal of this project is to convert the A-B remmeter to a new instrument with a sensitivity range from $0.025 \mathrm{eV}$ to several $\mathrm{GeV}$. This project on high-energy remmeters is also a collaborative program between the Institute of High Energy Physics (IHEP) in China and LBL, sponsored by the PRC/USA Joint Committee for Cooperation on High Energy Physics. In the agreement with IHEP, LBL designed the remmeter and carried out the high-energy experiments (above $20 \mathrm{MeV}$ ), while IHEP built some remmeters of LBL's design and make the low-energy tests $(0.025 \mathrm{eV}$ to $16 \mathrm{MeV})$.

\section{Accomplishments}

At LBL, the theoretical calculations for the extended remmeter were completed in 1992, and the results were verified by experiments (during 1992 and 1993) for medium- and high-energy tests, from $23.6 \mathrm{MeV}$ through $2.2 \mathrm{GeV}$. The results listed in Table 1 show that the deviations between experimental and theoretical calculations are less than $17 \%$, which is remarkably good.
Table 1. Comparison between the experimental and calculated results of the modified Andersson-Braun-type remmeter.

\begin{tabular}{|c|c|c|c|c|}
\hline \multirow[b]{2}{*}{$\begin{array}{l}\text { Test } \\
\text { No. }\end{array}$} & \multirow{2}{*}{$\begin{array}{c}\text { Beam } \\
\text { Energy } \\
(\mathrm{MeV})\end{array}$} & \multicolumn{2}{|c|}{ Ratio (Lead/Polyethylene) } & \multirow[b]{2}{*}{$\begin{array}{c}\text { Deviation } \\
(\%)\end{array}$} \\
\hline & & Experimental & Calculated & \\
\hline 1 & 2100.0 & 11.23 & 13.66 & 17.0 \\
\hline 2 & 1050.0 & 9.9 & 11.48 & 14.0 \\
\hline 3 & 1000.0 & 9.8 & 11.16 & 12.0 \\
\hline 4 & 400.0 & 8.9 & 10.21 & 13.0 \\
\hline 5 & 64.5 & 2.31 & 2.61 & 11.0 \\
\hline 6 & 50.0 & 2.38 & 2.22 & 7.0 \\
\hline 7 & 42.5 & 1.76 & 1.83 & 4.0 \\
\hline 8 & 40.0 & 1.8 & 2.22 & 18.0 \\
\hline 9 & 23.6 & 1.15 & 1.25 & 7.0 \\
\hline
\end{tabular}

The IHEP carried out the low-energy calibrations of the remmeter, mainly at the IHEP, Beijing University, in August 1993, with the participation of the principal investigator. The energies used were $0.025 \mathrm{eV}, 144 \mathrm{eV}$, $565 \mathrm{keV}, 2.2 \mathrm{MeV}, 4.4 \mathrm{MeV}$, and $16.67 \mathrm{MeV}$. In these calibrations, a standard Andersson-Braun remmeter was used to compare with the experimental models in the low-energy region, and the deviations between results with the standard and modified remmeter were well within $15 \%$ of the standard values. The experimental measured points for energies below $1 \mathrm{MeV}$ almost overlap, while data points above $1 \mathrm{MeV}$ from the test models are greater than those of the standard Andersson-Braun remmeter.

IHEP has manufactured four experimental remmeters following the principal investigator's design. All of them have been completed, with electronic counting systems attached to the polyethylene main body as a single unit. In addition, two of the remmeters have microprocessors for remote control, while the other two are built as standard high-energy neutron monitors. They look like standard commercial products, and can be used as practical prototypes after the final field test. 
The field tests of the fintal model are indispensable for completing this project. Though the Bevalac at LBL is no longer available for the monoenergetic highenergy n-beam experiment, other accelerator facilities are available for field tests of the renumeter. The results of Monte Carlc calculations, based on the energy spectrum of the n-beam, can still be useful to check the experin 'ntal results. It is expected that a brand new type of neutron remmeter, cylindrical, compact, and portable, could be available in the very near future.

In conclusion, the significance and value of this work can be summarized as follows:

1. The Monte Carlo method was useful for simulation of the neutron remmeter, and the theoretical calculations with the LAHET and MCNP codes have been verified experimentally and satisfactorily. This method proves to be a powerful and reliable tool in the design of a new neutron detector, e.g., the modification of a remmeter's response function for dose equivalents, according to the new quality factor recommended jointly by ICRP and ICRU.

2. The development of a neutron remmeter with its sensitivity range extended to energies of several $\mathrm{GeV}$ will produce a new and highly valuable instrument for nuclear science and high-energy physics. It would be useful for both reactor facilities and accelerator facilities worldwide, such as the ALS and other particle accelerator facilities, where the current $\mathrm{A}-\mathrm{B}$ remmeter is not able to measure neutron dose equivalent beyond $15 \mathrm{MeV}$.

3. The present experimental model of remmeter can be fully developed into a practical instrument for local-field monitoring or remote control and registration. With the sensitivity extended to the $\mathrm{GeV}$ range, this model would be a brand new type of neutron remmeter, compact and portable, and available in the near future. The demand for this new remmeter will be considerable.

\section{Publications}

R.K. Sun, G.F. Krebs, A.R. Smith, and H.H. Hsu, "A Neutron Dose Detector with REM Response to $1 \mathrm{GeV}$," presentation to the ANS/ENS 1992 International Conference, Chicago, Illinois, November 15-20, 1992.

H.H. Hsu and R.K. Sun, "Monte Carlo Calculations of High Energy Neutron Responses for Improved Andersson-Braun Remmeters," presented at the Joint Meeting of the American Physical Society, Washington, D.C., April 12-18, 1993.

R.K. Sun et al., "Experiments on the Energy Response of an High-Energy Neutron Monitor," a joint report of LBL and IHEP, August 1993.

R.K. Sun, "Annual Report of PRC/US Cooperative Program for 1992-1993, On the Development of an Extended Response Neutron Remmeter," internal memorandum LBL, October 5, 1993.

R.K. Sun et al., "Experimental Investigation of an Neutron Dose Detector With REM Response to $2 \mathrm{GeV}$," in preparation.

R.K. Sun et al., "Calibration of a High-Energy Neutron Remmeter in the Energy Range below $20 \mathrm{GeV}$," in preparation.

R.K. Sun et al., "The Performance of an EnergyExtended Remmeter and Its Comparison to a Commercial Andersson-Braun-Type Remmeter," in preparation.

\section{Patent}

Patent application: IB-930 High Energy Neutron Dosimeter DOE Case No.: S-76,656; RL-11867 Inventor: Rai-Ko Sun. 


\section{Information and Computing Sciences Division}

\section{Frameworks for Next-Generation Machining and Manufacturing}

Principal Investigator: Jane F. Macfarlane

Funding: $\$ 50,000$

Project No. 93009

\section{Project Description}

The goal of this project was to create a nextgeneration manufacturing information architecture that can integrate and augment the knowledge and data associated with a product description as the product travels through the design-to-manufacture cycle.

National competitiveness is of great concern in the U.S. today. American industries are openly asking for aid in combating the increasing competition for market share. This is not only due to increased competition from the world market, but also to a perceived decline in U.S. manufacturing.

Manufacturing practice on the manufacturing floor is a key component of this economic disease that has invaded U.S. companies. U.S. industries simply cannot compete in a global market if their time for design-to-market is considerably greater than their counterparts and their quality does not compare. It is the belief that innovative approaches to integrated manufacturing systems will allow the machine tool and manufacturing industries to recover a significant portion of market share.

Three essential elements of a next-generation manufacturing system were addressed. First, we designed a knowledge-based information architecture that captures many different knowledge and data representations associated with a product. Second, this information architecture was tied to an opensystem controller for machining. Third, high-speed networking and custom user interface designs were considered for making the information readily available to any user (designer to craftsman) through all stages of the design-to-manufacture cycle. These elements were designed and implemented for a commercial 4-axis machine tool and are being developed further, through UC Berkeley research funding, to allow easy implementation of specific technologies that are important to an intelligent machining environment.

\section{Accomplishments}

This year's work focused on two objectives. The first n'as to begin the development of a knowledge-based representation for capturing part and process through the LDRD program. The second, with support from UCB, was to develop a UNIX-based control system for the control of a Haas 4-axis vertical machining center with an automatic tool changer.

During this year, a prototype object-oriented system was developed that captures a part description as a collection of features. Within this framework, a feature based destructiye solid geometry (DSG) modeler was developed that ensures topological integrity of a designed part in the context of machining constraints. This methodology is designed to ensure manufacturability during the design phase by partitioning the machinability constraints into two types of feature interaction constraints: global feature interaction and local feature interaction. The approach during the design phase is to minimize global interactions and incrementally consider local feature interactions. This approach is described further in a invited paper that was presented at the ASME Winter Annual Meeting in November 1993.

Initially, the system automatically generated code that a previously developed expert system (Machinist) could use to determine part setups and process ordering. This first step demonstrated the integration of the part representations that are necessary to implement automated process planning. This information framework captures information about the part (features, dimensions, subfeatures etc.), the machining environment (machines and tools available), and the rules about process planning (embodied in the expert system Machinist). 
We have demonstrated the mappings between the DSG part representation-a collection of features and a stock, to a process plan representation-an ordered collection of setups and processes. The process plan representation is then mapped to a machining representation, a collection of machine tools and tools that can implement the processes. We are currently developing the mapping between the process plan and the part program in the open architecture controller environment. This involves a mapping from the machine tools and tool definitions to the Mosaic code necessary to implement the process. Initial demonstrations of this mapping have been completed.

Success in creating these methods and mappings will result in a fully integrated feature-based design system for rapid prototyping that will be the first of its kind. Providing an automated system that will map directly from a design representation to the machine code for creating the part on the machine tool within one integrated system is a unique approach to solving manufacturability problems and will result in a significantly reduced design-to-manufacture time. In addition, this system will have a direct effect on the capabilities, precision, and quality of current machine tools. The overall goal of our system is to demonstrate the effectiveness of highperformance information systems in improving manufacturing systems. It is intended to serve as a prototype that can be extended and enhanced by a broad community of computing system developers, sensor designers, control system engineers, and manufacturing technologists.

\section{Publications}

S.E. Sarma, P.K. Wright, and J.F. Macfarlane, "Reducing Global Feature Interactions: A New Paradigm for Simplifying Concurrent Process Planning," presented at ASME Winter Annual Meeting, New Orleans, LA, November 1993.

J. Stori, S. Sood, J.F. Macfarlane, and P.K. Wright, "A Knowledge-Based System for Micro-Planning in Feature Based Manufacturing," presented at ASME Winter Annual Meeting, New Orleans, LA, November 1993.

\section{POET: A Parallel Object-Oriented Environment and Toolkit for Enabling High-Performance Scientific Computation}

Principal Investigator: Jane F. Macfarlane

Funding: $\$ 81,800$

Project No. 93010

\section{Project Description}

The complexity of designing software solutions that can take advantage of high-performance computing platforms has grown significantly in the past few years. This has created a critical need for bridging the gap between the computer science necessary to algorithmically solve the problem and the representation of the scientific problem of interest. The purpose of POET (Parallel Object-oriented Environment and Toolkit) is to design and implement well-defined mappings between representations of physical phenomena in terms of mathematical structures and computational algorithms for modeling the phenomena on high-performance parallel computing platforms. The philosophy of the POET approach is to identify representations that solve entire classes of scientific problems. Thus, once the representation is defined for a particular problem area the mapping into the computing algorithm is handled automatically by the POET architecture. We have demonstrated the benefits of this approach by application to two scientific computing problems that currently are computationally limited by serial/vector computing hardware and software approaches.

The POET framework provides a structure for capturing the computational linkages necessary to solve specific scientific problem classes. Attached to the framework is the information about how to instantiate the linkages for a specific instance. A framework without a customization is a collection of static data structures that capture the knowledge about a specific problem domain. In this context, the framework captures the basic communication linkages and the structural aspects of the computational algorithm that are necessary to implement a parallel version of particular scientific problem classes. The purpose of this 
framework is to capture the intricacies of parallel communication and parallel algorithms for scientific problem classes in a predefined class hierarchy.

This approach does not attempt to create generalized problem solvers. What we capture is the computational linkages that are necessary to implement the problem, for example a partial differential equation solver, in a parallel environment. This parallel processing environment provides alternate computing mechanisms for improving performance. Rather than reprogramming the math packages to run in parallel environments, the approach is to recast the original scientific problem in a manner that will take advantage of the parallel environment. Essentially, we are exploiting the inherent parallelism of the physics itself. This representation is then captured in a framework that can be reused much in the same way that linear algebra packages were used in the past. In effect, the notion of a software library is being moved one level higher in abstraction with respect to the problem representation.

\section{Accomplishments}

We present specific applications to demonstrate two key results. The first is the design and implementation of an object class library and the associated infrastructure of POET object code that can span various applications. The second is to present results from these applications that show the resulting critical gain in compute power in the specific areas from which the applications were drawn.

For the first prototype application we targeted nextgeneration combustion models. Existing models for combustion are limited in the amount of chemistry that may be included in the model because of the computational requirements necessary to model the complex reactions associated with the combustion process. Current modelers are forced to use reduced chemistry models that approximate the 20 or more chemical mechanisms with 3 or 4 simple mechanisms. Parallel computing offers the only solution to advancing combustion modeling to accurately predict pollutant formation that results from the combustion process.

The results from this effort are shown in Figs. 1-3. Figure 1(top) shows the temperature profile that is generated by reduced chemistry models that are in general use today. Figure 1(bottom) shows the temperature profile generated by the POET code that includes full detailed chemistry. As can be seen from the figure, the profiles look similar and one would
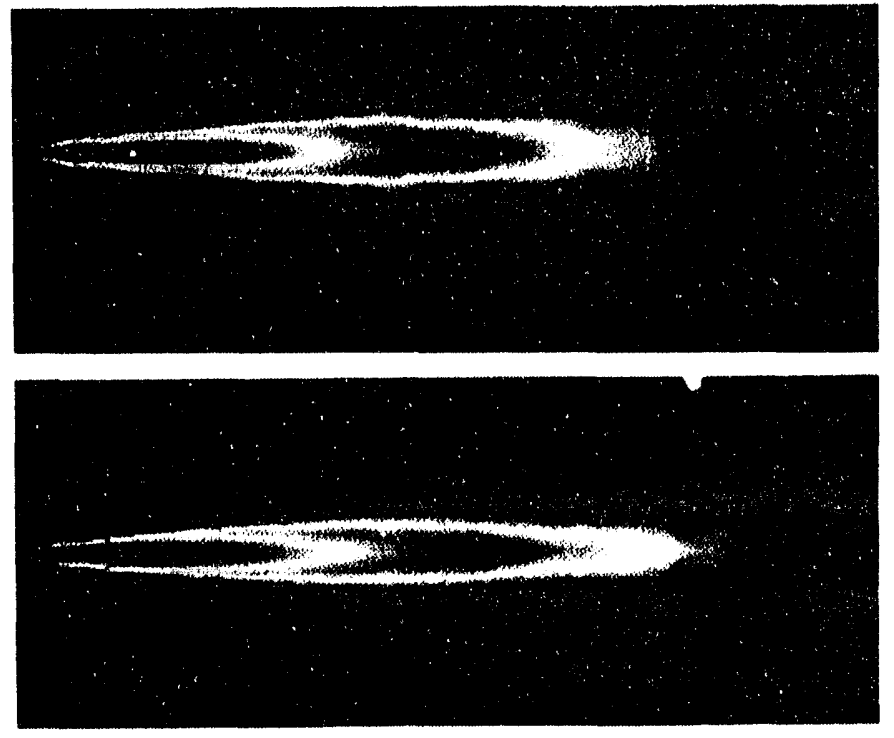

Fig. 1. Temperature profile calculated by a reduced chemistry model (top) and a detailed chemistry model (bottom). (Central region $=2100 \mathrm{~K}$; outer region $=300 \mathrm{~K}$.)

believe that the reduced chemistry model is effective at predicting the dynamics of the model. However, as can be seen in Fig. 2, the results for NO prediction are
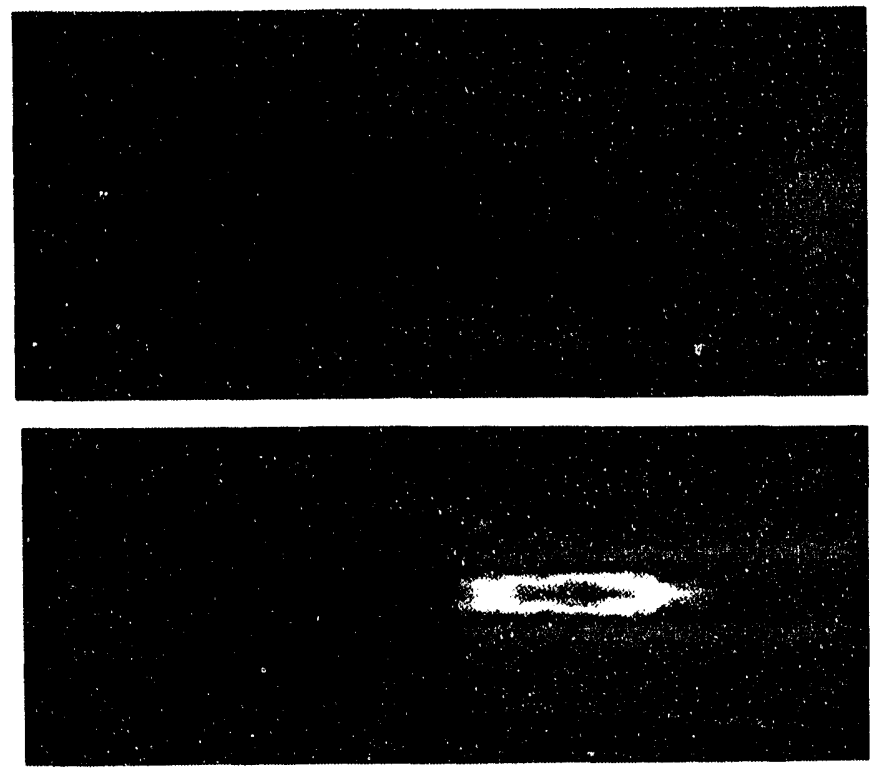

Fig. 2. NO concentration calculated by a reduced chemistry model (top) and a detailed chemistry model (bottom). (Central region $=50 \mathrm{ppm}$ mole fraction; outer region $=0$ ppm.) 


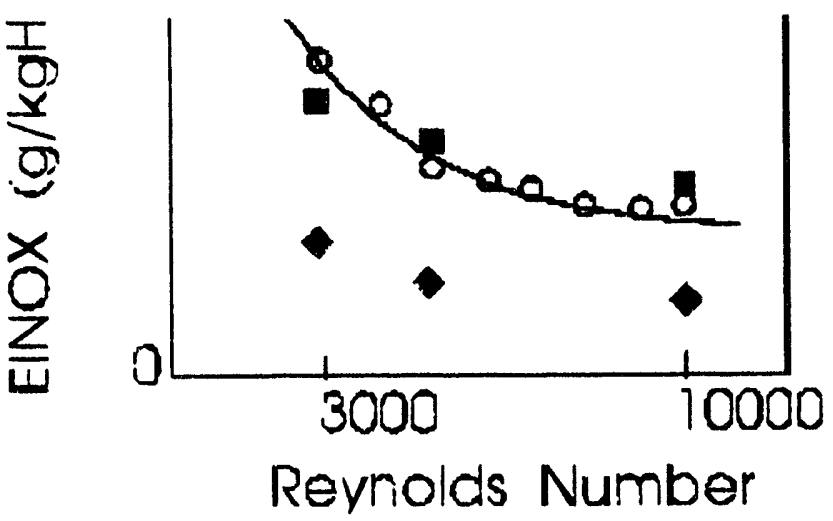

Fig. 3. NOx emission prediction comparison for experiment (O) [Driscall et al., Flame 88, 37 (1992)]; a detailed chemistry model (a) vs. a reduced chemistry model (A).

drastically different (more vivid in a false-color version). These results are crucial to the understanding of pollutant formation in combustion devices. The NO production is predicted at the parts per million (ppm) levels that combine from the many combustion devices in the environment to produce the severe environmental problems that we see today. Clearly, the reduced chemistry models fall short of allowing combustion scientists to understand the effects of the combustion process at the level necessary to design next-genera tion devices. The results are further demonstrated in Fig. 3, where experimental results are contrasted to the reduced chemistry model predictions and the detailed chemistry model predictions. This application has been easily moved to the following architectures: SGI, IBM RS600, and Sun $10 / 41$, without any modifications to the POET code.

Our second application targeted the human genome sequencing problem. Stepping up the discovery of human genome sequences is a major goal of the human genome project. Understanding the importance of a newly discovered sequence presents a major com puting challenge because exhaustive sequence comparisons, in which large databases of known sequences are searched for instances of the newly discovered sequence, already take a significant amount of computing resources. In addition, sequence length is increasing as the technology for recognizing the sequences improves. The result is genome databases that are growing at a very high rate and similarity searches that are becoming prohibitive in standard computing environments. POET provided a standard "bag-of-tasks" object in the architecture while concurrently providing a solution to the genome sequencing problem at hand. The technique provides a strategy for dealing with the exponential expansion of databases in the years to come.

The human genome application and the combustion modeling examples demonstrate the flexibility of the software design methodology captured in the POET software. The intelligence associated with distributing the problem over multiple processors is embedded in the objects defined in POET. As such, a user interface that is customized to the specific scientific application can provide easy access to supercomputing power to scientists who would otherwise be overwhelmed by the complexity of using supercomputing resources. With this added capability, problems that are currently unapproachable in these disciplines will be within reach, and we can begin to tackle problems that truly qualify as grand challenges.

\section{Publications}

J.F. Macfarlane and R. Armstrong, "Frameworks for Scientific Computation in a Parallel Environment," Cluster Computing Workshop, December 1993.

R.C. Armstrong, J.F. Macfarlane, R.E. Cline, and M.L. Koszykowski, "A Toolkit Approach to Distributed Computing," Proceedings of the Second Workshop on Cluster Computing, December 2, 1992.

J.F. Macfarlane, R. Armstrong, R.E. Cline, Jr., and M.L. Koszykowski, "Achieving Full Chemistry in Combustion Models Using POET," presented at the High Performance Computing Symposium-Grand Challenge Applications, Arlington, Virginia, March 19, 1993.

M.L. Koszykowski, R.C. Armstrong, R.E. Cline, Jr., J.F. Macfarlane, J.Y. Chen, and N.J. Brown, "ACME-the Advanced Combustion Modeling Environment," Computing at the Leading Edge: Research in the Energy Sciences, February 1993.

J.F. Macfarlane, R. Armstrong, R.E. Cline, Jr., and M.L. Koszykowski, "Application of Parallel ObjectOriented Environment and Toolkit (POET) to Combustion Problems," presented at the Hawaii International Conference on System Science, January 1993. 
M. Zorn, J.F. Macfarlane, and R. Armstrong, "BioPOET: A System for Large Sequence Analysis," DOE Genome Program Contractor and Grantee Workshop, Cold Spring Harbor, May 12-16, 1993.

M. Zorn, J.F. Macfarlane, S. Scherer, and R.

Armstrong, "Large Scale Sequence Analysis," DOE

Genome Program Contractor and Grantee Workshop, Cold Spring Harbor, May 12-16, 1993.

R.C. Armstrong and J.F. Macfarlane, "Achieving Full Chemistry in Combustion Models Using POET (Parallel Object-Oriented Environment and Toolkit)," presented at the NAS Parallel Tools Seminar, NASA Ames Research Center, January 27, 1993.

R.C. Armstrong, J.F. Macfarlane, R.E. Cline, M.L. Koszykowski, "Achieving full Chemistry in Combustion Models Using POET (Parallel ObjectOriented Environment and Toolkit)," presented at the National Energy Research Supercomputing Center MPP/Distributed Computing Seminar, December 18, 1992.

R.C. Armstrong, J.F. Macfarlane, R.E. Cline, and M.L. Koszykowski, "POET: A Toolkit for MIMD Parallel Computations," presented at the Lawrence Livermore Central Computing Seminar, October 13, 1992.

\section{Robust Shotgun DNA Sequence Assembly}

Principal Investigators: Frank Olken, Lawrence E. Lawler, and Daniel M. Gusfield

Funding: $\begin{array}{ll}\$ 51,500 \text { (FY 93) } & \text { Project No. } 92016 \\ \$ 82,900 \text { (FY 92) } & \end{array}$

\section{Project Description}

We are developing algorithms for DNA sequence assembly from shotgun data that address common sources of difficulty for current systems: repetitive DNA sequences, chimeric clones, and nonuniform error rates along fragments. Our work builds upon the four-phase approach of our collaborators J.

Kececioglu and E. Myers (see J. Kececioglu, "Exact and Approximation Algorithms for DNA Sequence Reconstruction," Ph.D. thesis, CS Dept., Univ. of Ariz. 1991), which decomposes sequence assembly into overlap detection, fragment orientation, fragment layout, and consensus sequence determination.

Discussions with $D$. Wemmer (LBL) suggested the problem of 2D nuclear magnetic resonance (NMR) spectral peak assignment could be addressed by graph theoretic methods akin to those used for sequence matching. We thus turned our attention to this problem.

The existence of 2D NOESY NMR spectral peaks signals the proximity of two nuclei (within $5 \AA$ ). The nuclei could be close either because they are contained in sequential residues in the protein sequence or because of the way protein folds. Such spatial constraints are used to compute the 3D structure of proteins. However, this requires that the NOESY NMR spectral peaks be correctly assigned to the appropriate pairs of nuclei. At present this is a very tedious manual process taking several personmonths. Attempts to automate the process have not been successful on large proteins. Typically, a 125 amino-acid protein sequence would generate 1-2 K 2D NOESY peaks.

\section{Accomplishments}

In FY 1992 we were concerned with data characterization, algorithm design, and acquisition of datasets and codes which we planned to modify or incorporate into a new sequence assembly system. See FY 1992 Progress report for a discussion of the algorithms for dealing with repetitive DNA sequences, chimeric clones, and nonuniform sequencing errors.

Our collaborators G. Alexander and T. Speed devised a maximum likelihood-based method for overlap detection and scoring, which accounts for substitution errors (but not repetitive DNA).

During FY 1993 a study was done on sequence data (from L. Hood and T. Hunkapiller), as well as testing and fitting statistical models for the sequencing errors. The analysis indicates that incorrect reporting of the length of short runs of single letters (e.g., runs of $A^{\prime}$ s) accounts for about 75 percent of the sequencing errors. An EM algorithm has been used to estimate model parameters. Approximate likelihoods for multiple sequence alignments have been calculated based on this error model. An advantage of this approach is that most insertion/deletion errors can be modeled as substitution errors over an extended alphabet, i.e., with additional symbols for runs. This work will be reported in Alexander's forthcoming thesis. 
In the 2D NMR problem, the NOESY peaks can be grouped (by means of perturbed TOCSY experiments and related analyses) into spin systems that correspond to the individual residues (amino acids) of the protein sequence. The difficulties lie in determining which spin systems correspond to which residues in the protein sequence.

There are 3 major sources of difficulty:

(1) Often, we can not classify a spin system into exactly one type of amino acid, but only an aminoacid equivalence class (typically containing 4 possible AAs).

(2) Peaks indicating coupling between spin systems may be due to either sequential or nonsequential residue proximity. Only peaks indicating sequential residues are relevant for matching the protein sequence residues to spin systems.

(3) A pair of spin systems may be degenerate (superimposed peaks), so that we may not be able to determine which peaks (indicating spin systems proximity) couple to which spin systems.

Thus, leaving aside errors, spectral peak assignment can be viewed as finding a maximum likelihood consistent embedding of the linear protein sequence graph (PSG) in the (ambiguous) aggregated spin system proximity graph (ASSPG).

Such an embedding can be found by a relaxation to a shortest path problem in the correspondence graph (defined on the cross product of the amino-acid residues and the spin systems). Fast algorithms are known to solve shortest path problems. A branchand-bound procedure (deleting offending nodes of the correspondence graph) will be used to enforce the constraint that the embedding of the PSG in the ASSPG be one-to-one on the respective vertices.

A variety of extensions of this model can be made to incorporate various sorts of errors (estimated by $D$. Wemmer): missing edges in the ASSPG (missing peaks) $(5 \%)$, missing spin systems $(2 \%)$, ambiguous or incorrectly resolved degenerate spin systems (3\%), misclassification of spin systems ( $3 \%$ unless larger equivalence classes are used).

The success of this approach hinges on the amount of ambiguity and errors in the experimental data. We are designing computational experiments to empirically validate the approach. 


\section{Life Sciences Division}

\section{Biophysical Modeling for Optimization of Treatment Plans}

Principal Investigators: William T. Chu and Bernhard A. Ludewigt

Funding: $\$ 94,900$

Project No. 93011

\section{Project Description}

Heavy-charged-parti-le beams offer physical and biological advantages for the treatment of human cancer over conventional radiation. Due to their welldefined range and the Bragg ionization curve characteristics, the highest dose can be deposited in the target volume by properly adjusting the energy of the particles. Heavy particles suffer less multiple scattering than conventional radiation that leads to smaller lateral dose falloffs (penumbrae) and, perhaps even more importantly, opens up the possibility of scanning the target volume in three dimensions with narrow pencil beams.

Pencil-beam scanning is not only an excellent method for conforming the high dose region to the target volume but also provides for a new flexibility in dose delivery. Currently, the highest uniform dose is given to the target volume while not exceeding limits to the surrounding normal tissues. By employing pencilbeam scanning dose distributions can be arbitrarily shaped in three dimensions, limited only by the width of the pencil beam. This capability can be exploited for optimizing the biological effect, i.e., the tumor control probability and the normal tissue complication rate. Depending on the radiosensitivity of the surrounding organs and other normal tissues and tumor characteristics, a sharper or a wider distal falloff may be more advantageous; it may be best to deliver nonuniform fields from various directions, which in combination result in the optimal dose distribution.

In order to take full advantage of the pencil-beam scanning capabilities, new and more automated treatment planning techniques must be developed. The goal of this study was to explore concepts for optimizing three-dimensional treatment plans and, specifically, to develop algorithms and methods for the optimization of scanning patterns that can be incorporated into treatment planning software.

A stepwise approach was taken towards the goal of optimizing treatment plans. First, algorithms for solving the inverse problem of optimizing threedimensional dose distributions were tested, and, second, the nonlinear problem of directly optimizing the cell survival in the target volume and surrounding tissues was studied before addressing the optimization of tumor control probabilities and normal tissue complication rates. All calculations were based on a superposition of pencil beams reflecting a realistic beam-scanning system. The result of the optimization procedure, the weights for each pencil beam, or the Bragg peak density function, correspond to a scanning pattern that can directly be used to control the scanning system. The same mathematical formalism can also be employed for handling a more general class of problems where beams or radiation fields can be described by a superposition of narrow pencil beams. In fact, this method has been used in dose calculations when the differences in multiple scattering and energy losses, for example between bone and s.jt tissue, were investigated.

\section{Accomplishments}

Full 3-D optimizations were performed on a $128 \times$ $128 \times 40$ matrix for several test volumes. The calculations were done on a 1-mm transverse grid with a 5mm longitudinal spacing. The modeled pencil beam includes all features essential for the purpose of this study: change in stopping power as a function of depth, small-angle scattering, energy straggling, and losses due to nuclear interactions. The spread-out Bragg peak (SOBP) requires beams of several energies to be overlaid to produce a smooth longitudinal dose profile and to minimize the entrance dose. Scattering spreads the beam, and nuclear interactions fragment the primary particles and attenuate the beam along its path. At present the dose deposited by the fragments has not been taken into account. To facilitate the simulation calculation, the energy deposition function and rms beam width of the pencil beams over the entire 
energy range are precalculated. The programs for the beam modeling and the optimization procedure were written in $C$ and in FORTRAN and run on Sun SPARC stations.

Displayed in Fig. 1 are the results for a test volume with the shape of half a ring wrapped around a sensitive structure, for example, a spinal cord. Shown are distributions on a cut through the volume in the main plane of the ring. The beams enter from the side opposite the "cutout" for the sensitive structure (i.e., upper right). Fig. 1(a) shows the desired fractional cell survival. Inside the target volume the desired cell survival probability was specified as $50 \%$ and outside as $100 \%$. For better visualization the survival distributions in Fig. 1 are inverted. Figure 1(b) displays the cell survival distribution optimized using the iterative algorithm. This distribution represents the "best" one, which is achievable using the given pencil beams. The fractional cell survival is about $50 \%$ in the target volume and significantly higher in the entrance region. The sensitive volume at the distal end is spared. The Bragg peak density is shown in Fig. 1(c). The beams that stop at the edges of the target volume have the highest weights. This is a result of the desire for a sharp penumbra and for the sparing of the normal tissue while keeping the survival within the target volume at or under the desired level.

This study has confirmed that the iterative method is not only well suited for optimizing dose distributions, but also converges when used for solving the nonlinear problem of optimizing cell survival distributions. Most importantly, it has been demonstrated that a fully three-dimensional optimization can be done on available workstations, e.g., Sun SPARC stations 2 and 10. Between 20 and 50 iterations are required for a satisfactory optimization, which can be performed in less than a day on a SPARC station 10. This study has clearly demonstrated the feasibility of incorporating optimization procedures based on an iterative algorithm for solving the inverse problem into the treatment planning process.

The authors would like to thank John Staples for helpful discussions.

\section{Publications}

W. Chu, "Conformal Treatment Planning," presented at the XIX Proton Therapy Cooperative Group Meeting, Cambridge, MA, October 31-November 2, 1993.

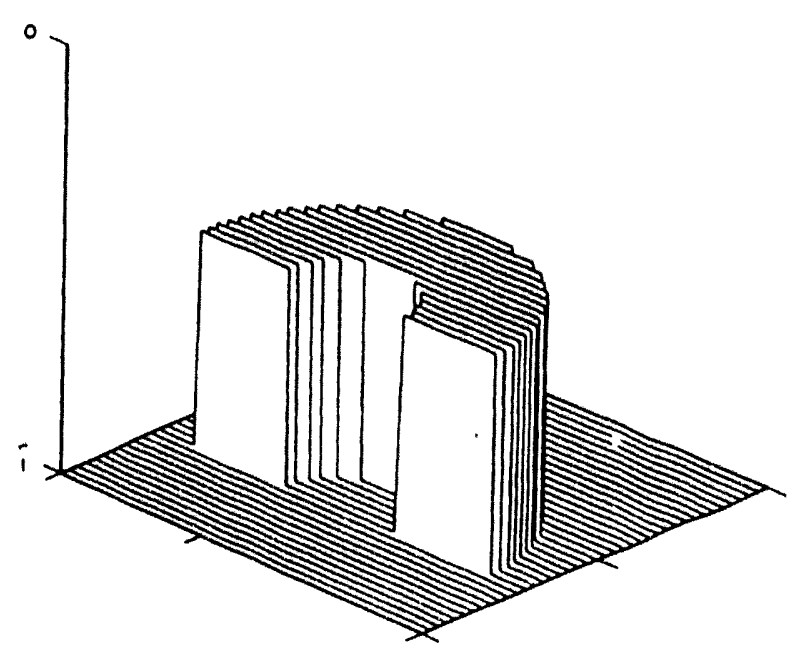

Fig. 1(a). Desired fractional survival.

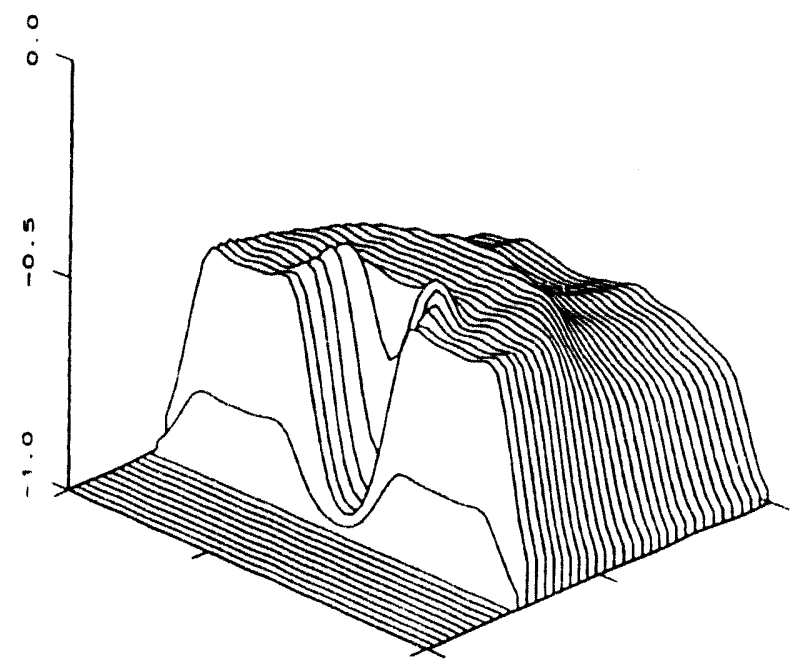

Fig. 1(b). Optimized fractional survival.

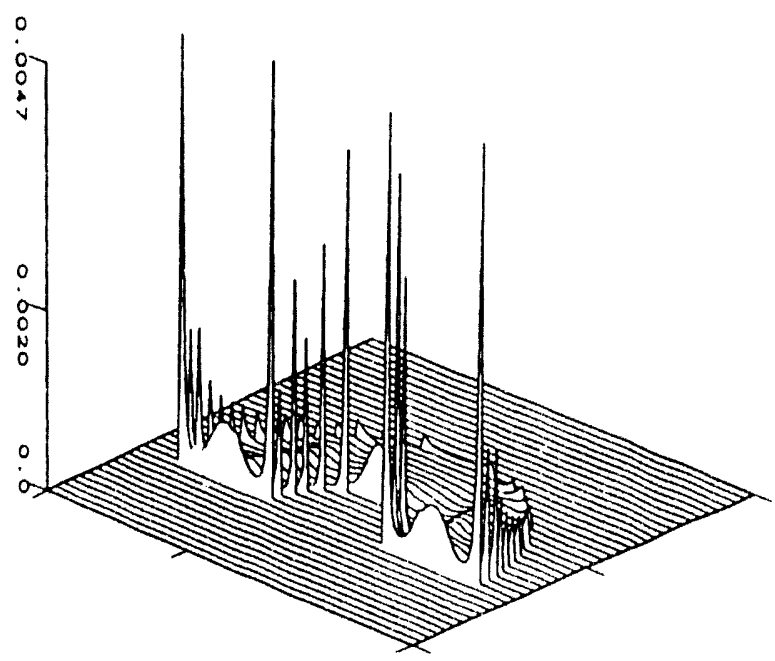

Fig. 1(c). Bragg peak density. 


\section{Environmental Air Pollutants and Oxidative Stress}

Principal Investigators: Trudy M. Forte and Ronald
M. Krauss

Funding: $\$ 87,000$ (FY 93)

$\$ 90,500$ (FY 92)

Project No. 92019

\section{Project Description}

The overall objectives of this research are (1) to study the effect of environmental pollutants such as ozone, reactive aldehydes, and free radical generators on plasma lipoproteins and (2) to determine whether specific antioxidants can protect plasma components, particularly lipoproteins, from oxidative stress. In the past year we compared gas phase cigarette smoke (contains high levels of reactive aldehydes) with copper ions (free radical generator) with respect to their ability to inhibit lecithin:cholesterol transferase (LCAT) activity and to modify lipids and/or vitamin $E$ levels in lipoproteins. Comparison of gas phase of cigarette smoke (CS) effects with those of a known free radical generator can provide insights on how cigarette smoke inhibits LCAT and/or modifies lipoproteins.

\section{Accomplishments}

Environmental air pollutants such as those found in gas phase of CS have the potential ability to diffuse across the epithelial lining of the lung and dissolve either in the lung interstitial fluid or the plasma of capillaries in the lung. The oxidative components generated by cigarette smoking may alter lipoproteins and other components of lipoprotein metabolism that will ultimately result in increased risk for cardiovascular disease. Oxidative modification of lipoproteins can in fact be an important pathophysiological contributor to foam cell formation in the artery wall. In the past year we carried out extensive studies to determine the mechanism(s) by which CS alters LCAT activity. In addition, we examined several antioxicants for their ability to protect LCAT activity. Since copper ions have frequently been used to oxidize lipoproteins and since its mode of action is known, we compared CS effects with those of copper ions.

Pooled plasma obtained from normal subjects was dialyzed against phosphate-buffered saline and exposed to 1 puff of filtered CS (U. Kentucky 2R1 cigarettes) at $15 \mathrm{~min}$ intervals for periods up to $6 \mathrm{hr}$
( 1 cigarette was used every $2 \mathrm{hr}$ ). Since oxidative constituents in CS are numerous and complex, we also examined the properties of a well-known prooxidant, copper $\left(\mathrm{Cu}^{++}\right)$, on LCAT and compared its effect with CS exposure. Representative results, shown in Fig. 2, indicate that $\mathrm{CS}$ and $\mathrm{Cu}^{++}(500 \mu \mathrm{M}$ added to plasma) had similar effects on LCAT activity that shows a substantial reduction (approximately $30 \%$ ) as early as $15 \mathrm{~min}$ of exposure. These results suggest that LCAT activity is extremely sensitive to oxidative damage by either CS or copper since inhibition is extensive at $15 \mathrm{~min}$.

LCAT is unique because, in addition to catalyzing the esterification of cholesterol, it also has the ability to hydrolyze phospholipid (PC) in an aqueous environment. This phospholipase activity may have an important role in the normal metabolism of low-density lipoprotein (LDL) and high-density lipoprotein (HDL). We asked the question whether phospholipase activity was also altered by oxidizing events, and we found that both $\mathrm{CS}$ and $\mathrm{Cu}^{++}$rapidly inhibit phospholipase activity. Oxidation events, therefore, are not restricted to the esterification function of LCAT.

LCAT is a highly glycosylated protein of approximately $66 \mathrm{kD}$, which possesses two disulfide bridges and two free cysteines (sulfhydryls). The catalytic site is believed to involve the Ser-His-Asp triad that is located in a so-called pocket bounded by the two cysteines. It is reasonable to expect that $\mathrm{CS}$ and $\mathrm{Cu}^{++}$ may oxidize the free cysteines of LCAT, thus forming a disulfide bridge in its functional region. This being the case, it is likely that antioxidants (or reducing agents) that protect thiol groups may protect LCAT

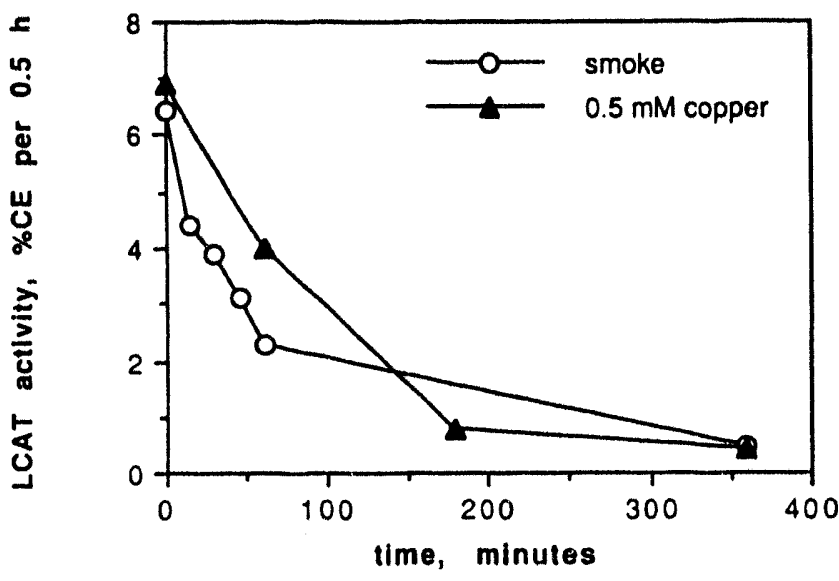

Fig. 2. Effect of cigarette smoke and copper on LCAT activity. 
activity. To test this hypothesis, glutathione, a wellknown protector of thiols, was added to the plasma prior to exposure to smoke. At $1 \mathrm{mM}$ glutathione, there was $75 \%$ protection after 3-hr exposure to CS. These data suggest that preservation of the free cysteine groups are probably essential for maintaining LCAT activity. It is likely that CS leads to the oxidation of the free thiols after plasma protein thiols are spent by exposure to $C S$.

We also investigated the effect of $\mathrm{CS}$ and $\mathrm{Cu}^{++}$on polyunsaturated fatty acid levels (20:4 relative to $16: 0$ ) and found that $\mathrm{Cu}^{++}$decreased the polyunsaturated fatty acids approximately $11 \%$ for HDL and $15 \%$ for LDL after 6-hr exposure. CS had no effect on the polyunsaturated fatty acids. CS, however, had a much more profound effect on vitamin $E$ levels of HDL and LDL where vitamin E completely disappeared after 6-hr exposure compared with $\mathrm{Cu}^{++}$. In the latter case, $29 \%$ and $33 \%$ vitamin $E$ remained in HDL and LDL, respectively (see Fig. 3), after 6-hr exposure.

The results suggest that components of CS and copper-mediated lipid peroxidation products are potent inhibitors of LCAT. Oxidative processes, especially thcse mediated by CS, may interfere with normal HDL maturation and the reverse cholesterol transport process. The depletion of vitamin $\mathrm{E}$ by CS may also have a signiicant role in increasing the oxidation events associated with HDL which is the key substrate for LCAT. Inhibition of the reverse cholesterol transport process, which requires both HDL and LCAT, by environmental air pollutants may contribute to increased risk for cardiovascular disease.

\section{Publications}

M.R. McCall, J.J.M. van den Berg, F.A. Kuypers, D.L. Tribble, R.M. Krauss, L.J. Knoff, and T.M. Forte, "Modification of LCAT Activity and HDL Structure: New Links between Cigarette Smoke and CHD Risk," Arteriosclerosis and Thrombosis, in press.

J.K. Bielicki, M.R. McCall, J.J.M. van den Berg, F.A. Kuypers, and T.M. Forte, "Inhibition of LCAT and Modification of HDL in Plasma by Copper Ions and Gas-Phase Cigarette Smoke," in preparation.

M.R. McCall, F. Kuyper, J. van den Berg, R.M. Krauss, L. Knoff, D.L. Tribble, and T.M. Forte, "Gas Phase Oxidants from Cigarette Smoke Deplete Plasma

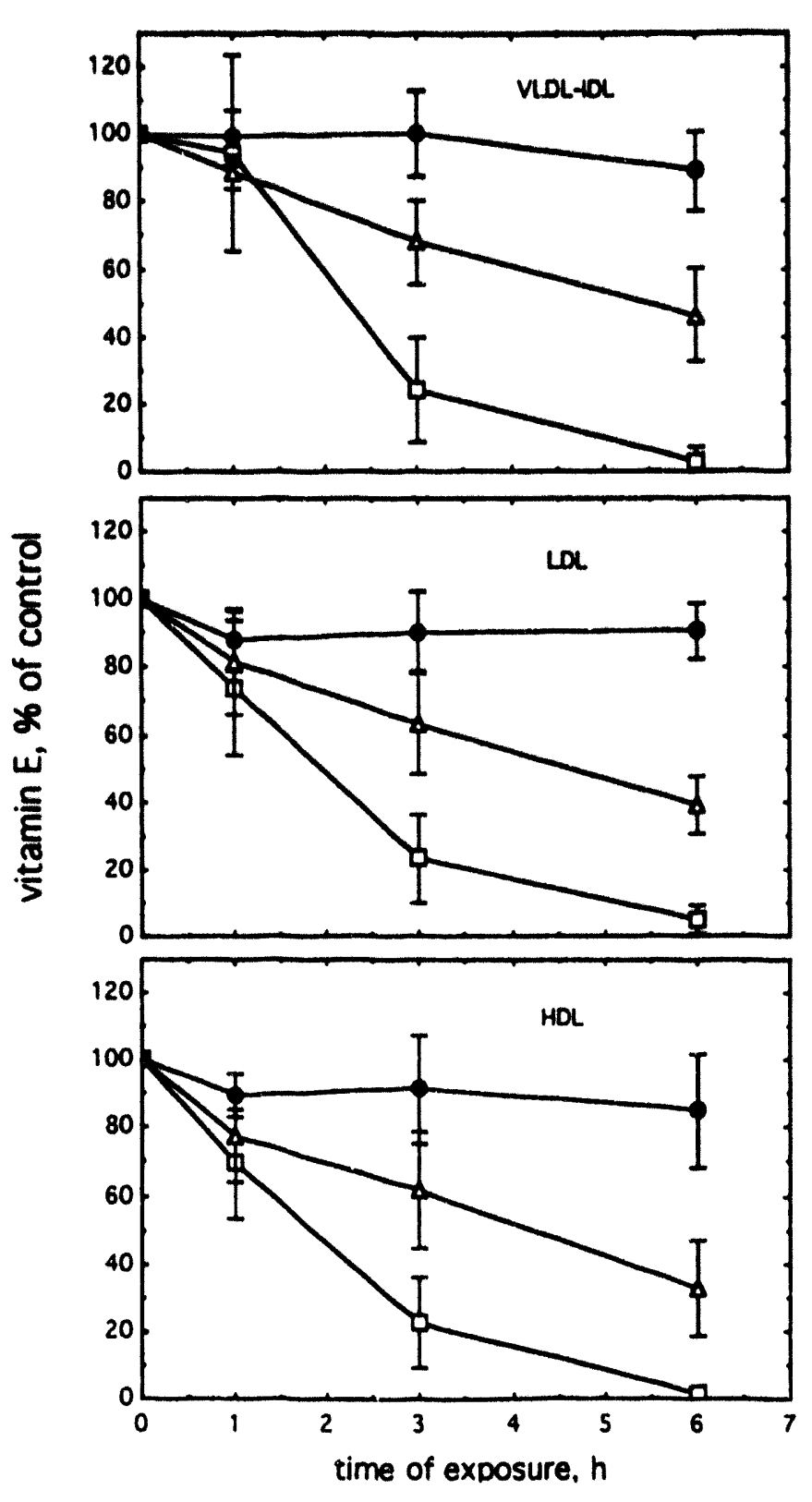

Fig. 3. Changes in vitamin $E$ in lipoprotein fractions after exposure to cigarette smoke and copper: $\bullet=$ air, $\Delta=$ copper, $\square=$ smoke.

Vitamin E and Impair LCAT Activity," FASEB J. 7, Part 1, A414 (1993).

J.K. Bielicki, M.R. McCall, J.M. van den Berg, F.A. Kuypers, and T.M. Forte, "Inactivation of LCAT and Modification of HDL in Plasma by Oxidative

Processes," Am. Heart Assoc. 66th Scientific Sessions, November, 1993. 


\section{A Comprehensive Approach to Protein Folding}

\author{
Principal Investigator: Teresa L. Head-Gordon \\ Funding: $\$ 98,800$ (FY 93) \\ $\$ 10,800$ (FY 92) \\ Project No. 92021
}

\section{Project Description}

The realization of a solution to the protein-folding problem would include such benefits as the reengineering of defective proteins indicted in disease, the design of synthetic proteins relevant for biotechnical applications, and a general understanding of how molecules self-assemble. A detailed understanding of the kinetic mechanism of the entire folding event would comprise a complete solution, but the complexity of the problem ensures that such a solution will remain elusive in the near future. Therefore researchers generally take one of two routes in protein folding: (1) the development of empirical predictive mappings between amino acid sequence and tertiary structure or (2) the use of simplified models in the hope of gaining greater physical insight. The former approach has the advantage of genuinely predicting structure, but is empirical and subject to uncontrolled error. Physical models are meant to be robustly predictive in a general sense, but are often so greatly simplified that they have not yet yielded genuine structures that can be directly compared with experiment. This research approach involves an emphasis on model studies whose purpose is to converge with the goal of quantitative protein structure prediction.

Therefore, a tandem approach of using simple models for insight in order to develop folding algorithms that provide quantitative protein structure prediction is the research aim. A constrained optimization algorithm known as the Antlion method was developed and has been used with success on the 26residue polypeptide, melittin. While the melittin pilot study demonstrates that constrained optimization provides the framework for a predictive atomic structure algorithm, it also reveals the algorithmic components that are weak: (1) an understanding of the chemistry that drives folding to the area of the vast potential surface where the native minimum is found; (2) once in this area, predicting the final native structure adopted; (3) incorporating information from (1) and (2) as robust constraints on a derived energy function to predict the native structure minimum with atomic resolution, and (4) progressing toward a derived energy function that is quantitative. To address these weaknesses, three distinct areas of research were set up. The first is an understanding of the initial folding kinetics in order to discern what chemistry - the amino acid sequence, aqueous environment, or both-dominate the drive toward native structure. The second research area begins with the assumption that once initial folding is completed (regardless of the mechanism), the chemical details of the amino acid sequence then dictate the final folded outcome. Here neural networks are used as a computational tool to make this final prediction, and they will eventually use the knowledge of the initial folding kinetics to further improve their predictions. Finally, the quantitative predictive problem was tackled by studying small amino acids with the best theoretical methods currently available. Once these research areas yield fruit, their incorporation into constrained optimization should comprise a robust and accuratr. prediction algorithm for protein structure prediction.

\section{Accomplishments}

\section{Early Folding}

The domination of aqueous environment over sequence in determining the early folding events would be consistent with a hydrophobic interaction mechanism. One fundamental question that has not been addressed until now is what is the molecular origin of the hydrophobic interaction and effect. We have developed a new statistical mechanical orientation perturbation theory for pure liquid water and water solutions, which will permit the resolution of the exact nature of the hydrophobic interaction. Preliminary studies have now been completed on the association of small, spherical nonpolar solutes in water to determine what distinguishes water from other organic solvents-its small size or hydrogenbonding capabilities-a distinction which is still under debate, but which will be resolved at the completion of this study. Also, a new Monte Carlo algorithm was developed that improves convergence of these calculations. A substantial grant of Cray time has been received to move on to understanding more complex solutes and their association in water.

\section{Final Native Structure}

The successful application of neural network algorithms for prediction of protein structure is stymied by three problem areas: (1) the sparsity of the database of known protein structures; (2) poorly 
devised network architectures that make the inputoutput mapping opaque; and (3) a global optimization problem in the multiple minima space of the network variables. As a consequence, the current protein structure predictive capacity of neural networks is no better than sequence homology modeling and statistical analyses of the databank of structures-methods that have not in themselves yielded reliable prediction algorithms. We propose to correct these problems by focusing on well thought out network designs. Recently completed is a neural network design for prediction of amino acid contacts of a simplified polypeptide model residing in two dimensions, with only two amino acid types- $A$ and B. The small size and reduced dimensionality allows the determination of the global energy structure for all possible sequences of pentamer, hexamer, and heptamer lengths. Given this complete structural database, neural networks were devised that reproduce the "tertiary" structure of all sequences with absolute accuracy and with the smallest number of network variables. These optimal networks reveal that thoughtful network designs can actually overcome the detrimental traits described above to provide network algorithms which genuinely impact on the ability of the network to generalize or learn the desired mappings. Furthermore, the 2D polypeptide model shows sufficient chemical complexity so that transfer of certain aspects of neural network technology to more realistic 3D proteins is evident. We are now at the stage of transferring some of the learned insights of this simple network to networks that predict protein structure of large lengths with full sequence diversity. We are also scaling up our polypeptide model to include more chemical complexity. Air Force Office of Scientific Research fund- , ing was recently received for three years to further develop this area of neural network research.

\section{Quantitative Energy Surfaces}

Small peptides are ubiquitously used as structural models of larger proteins, especially in the design of protein force fields for theoretical protein structure determination. Whether such small peptides are good structural models is open to question. We can begin to address this concern by interrogating the conformational preferences of these small peptides in gas and solution phases with the best theoretical means possible. $\mathrm{Ab}$ initio electronic structure methods are known to be quantitatively correct when appropriate levels of theory are used. Using the power of these theoretical tools, we have recently evaluated the full conformational space of glycine and alanine dipeptide in their gas and solution phases (the latter represented as a dielectric continuum). One important conclusion found from these dipeptide studies is the lack of secondary structure minima in the gas phase for these two peptides. We have recently finished electronic structure calculations that demonstrate that right and left-handed $\alpha$-helical secondary structure stabilization in glycine and alanine dipeptides is a solvent-induced effect; this origin of secondary structure is a surprise and strongly demands further improvement for incorporating environment in polypeptide structure studies. However, the quantitative theoretical tools currently lack the ability to accurately handle solvent environments.

\section{Publications}

H.S. Shang and T. Head-Gordon, "Stabilization of Helices in Glycine and Alanine Dipeptides in a Reaction Field Model of Solvent," J. Am. Chem. Soc., in press.

T. Head-Gordon, "Toward Quantitative Protein Structure Prediction," in Protein Structure Determination, K. Merz and S. LeGrand, eds., Springer-Verlag, in press.

T. Head-Gordon and F.H. Stillinger, "Optimal Neural Networks for Protein Structure Prediction," Phys. Rev. E 48, 1502 (1993).

F.H. Stillinger, T. Head-Gordon, and C.L. Hirschfeld, "Toy Model for Protein Folding," Phys. Rev. E 48, 1469 (1993). 


\section{Organometallic Complexes as Novel DNA Sequencing Agents}

Principal Investigator: Marcos F. Maestre and

Richard H. Fish

Funding: $\$ 50,000$ (FY 93)

$\$ 87,400$ (FY 92)

Project No. 92022

\section{Project Description}

The sequencing of the human genome is very laborious and time consuming by virtue of its enormous size. A technique, which would shorten the time and/or reduce the effort in any of the stages of sequencing the DNA of any one of the chromosomes, would be of considerable utility in the human genome program. In this project, the recently developed techniques for visualizing and manipulating single DNA molecules by fluorescence microscopy was used to manipulate a single DNA molecule in the microscope stage. The main focus of the project was to develop a series of water soluble organometallic rhodium and ruthenium complexes that can specifically bind to defined sequences in oligomers. These organometallic-oligomer complexes will then be bound to DNA strands by hydrogen bonding. This organometallicoligomer-DNA complex was used as an anchor to given metal supports such as gold or platinum surfaces. The bound organometallic-oligomer-DNA complex will be stretched by applying electric or magnetic fields. The directions of stretching the DNA molecule was controlled to allow the organometallicoligomer complexes, each with different sequences, to bind the various segments of a chosen DNA strand to the metal supports, a type of "stitching." This binding to DNA strands by the organometallic-oligomer complex was selective by choosing the correct sequence of the oligomer section of the anchoring organometallic complex.

There are three important aspects to this project: (a) The use of water soluble organometallic rhodium and ruthenium complexes to study their binding characteristics to oligomers. This is a necessary condition, due to the liability of the DNA molecules in organic solvents. In this regard, a $\left[\mathrm{Cp} * \mathrm{Rh}\left(\mathrm{H}_{2} \mathrm{O}\right)_{3}\right]^{2+}$ complex can be prepared and is readily water soluble. (b) Selective binding of the organorhodium and organoruthenium complex to DNA bases. The $\left[\mathrm{Cp} * \mathrm{Rh}\left(\mathrm{H}_{2} \mathrm{O}\right)_{3}\right]^{2+}$ complex has been shown in preliminary ${ }^{1} \mathrm{H}$ NMR experiments to selectively bind to adenosine (N7 and N2) and guanosine (N7), but not thymidine or cytosine. Thus, the important criteria of selective binding to DNA oligomers of a defined sequence is now possible. (c) The organometallicoligomer complex can now be ligated to the specific sequence in the DNA molecule. It is important that the organometallic-oligomer-DNA complex also be bound to the specific supports; preferably, metallic surfaces of the microelectrodes such as gold or platinum surfaces. This also is entirely feasible, since open coordination sites on the rhodium metal center should be available for this type of additional binding.

\section{Accomplishments}

\section{Manipulation of Single DNA Molecules}

A short description of the new methods for manipulation of single DNA molecules follows: The rationale for the invention is to construct a network of electrodes of dimensions of the order of the size of large DNA molecules. This would be dimensions in the range of 20 to $50 \mu \mathrm{m}$ in separation with electrodes sizes of approximately 10 to $20 \mu \mathrm{m}$ in thickness. The second important concept is the use of inhomogeneous electrical fields. If the long DNA polymers are to be manipulated in a way so that different sections of the molecule can be placed in specific position sin the electrode net, it is essential that these parts feel different forces, i.e., different electrostatic fields. By varying the voltage between the different electrodes (by computer control) the molecule can be oriented and moved in the plane of the electrode net. In this manner, it can be placed in regions where it would be accessible to chemical modification.

\section{Sequence-Specific Oligonucleotides}

Since the described application depends on the selective binding of the sequence-specific oligonucleotide to the [Cp* $\mathrm{Ch}$ (Aqua)] complex, we stressed these studies over the above-described adenine dimer and tetramer oligomer studies. Structural studies on several sequence-specific oligonucleotides that may selectively interact with the [C $\mathrm{p}^{*} \mathrm{Rh}$ (Aqua)] complex; for example, $\left(3^{\prime}-\mathrm{dA}-5^{\prime}\right) 12$

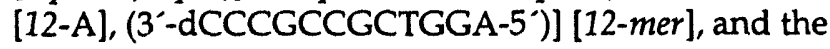
(3'-dA)12(dCCCGCCGCTGGA-5') [24-mer]; were analyzed by $C D, U v$-vis, and GFAA ( $R h)$ analysis, after purification by dialysis.

A comparison of the $C D$ spectra of the 24-mer, 12-mer, and the 12- $A$ oligomers, when reacted individually with the [Cp*Rh(Aqua)] complex at the molar ratios of one $C p^{*} R h /$ two base pairs, show that the greatest 
change in the $C D$ corresponds to the regions of the $C D$ spectrum that are associated with the 12-A oligomer structure. Interestingly, the largest alteration of the $C D$ is found in the spectral regions below $260 \mathrm{~nm}$ in the 24-mer, and similarly in the 12-A oligomer. There are only small changes in the 12-mer spectra upon addition of the [Cp*Rh(Aqua)] complex. The $C D$ spectral studies are, therefore, indicative of changes mainly in the 12- $A$ oligomer section of the 24mer. This agrees with the previous NMR binding studies of the [Cp*Rh(Aqua)] complex with the individual nucleobases, which indicated a strong binding preference for the adenine base.

The CD spectral changes in the 24-mer upon reaction with $\left[C p^{*} R h(\right.$ Aqua)] are indicative of preferred binding to the adenine rich section of the molecule, i.e., the $12-A$ section of the 24-mer. While we do not as yet know the exact bonding mode of the 24-mer with the $C p^{*} R h$ group from these $C D$ results, i.e., hydrogen, electrostatic, or covalent bonding (or all three), complementary UV-vis and GFAA analyses to determine the ratio of $\mathrm{Rh} /$ nucleobase were initiated. After extensive dialysis $(21 \mathrm{~h})$ of the $\mathrm{C} \mathrm{p}^{*} \mathrm{Rh}-24-$ mer complex, to determine the extent of covalent bonding, the radio of $0.6 \mathrm{Rh} / 24$-mer was found. We conclude from these preliminary results that all three types of possible bonding modes may be present (concentration dependent) with the covalent bonding associated with the terminal 3 -adenosine base in the 12- $A$ portion of the 24-mer as also suggested from our ApA NMR results. Further degradation and HPLC isolation studies with the Cp*Rh-24-mer complex will be attempted to ascertain terminal binding to the $3^{\prime}$-ApA position. The conclusion from these investigations is that there is preferred binding of $\mathrm{Cp}^{*} \mathrm{Rhodium}$ to specific runs of poly $A$ in an oligomer, and this could be used as the binder to the metal or glass substrate.

\section{Publications}

D.P. Smith, E. Baralt, B. Morales, M.M. Olmstead, M.F. Maestre, and R.H. Fish, "Bioorganometallic Chemistry. 1. Synthetic and Structural Studies in the Reactions of a Nucleobase and Several Nucleosides with a ( $\mathrm{h}^{5}$-Pentamethylcyclopentadienyl)rhodium Aqua Complex," J. Am. Chem. Soc. 114, 16047 (1992).

D.P. Smith, M.M. Olmstead, M.F. Maestre, and R.H. Fish, "Biooganometallic Chemistry. 2. A Synthesis and Structural Study of the Reaction of a Nucleobase, 1-Methylcytosine, with a ( $\mathrm{h}^{5}$-Pentamethyl- cyclopentadienyl)rhodium Aqua Complex," Organometallics 12, 593 (1992).

D.P. Smith, E. Kohen, M.F. Maestre, and R.H. Fish, "Biometallic Chemistry. 3. The Role of the Phosphate Group During Reactions of Adenosine Monophosphate Derivatives with a ( $h^{5}$-Pentamethylcyclopentadienyl)rhodium Aqua Complex, in the Diastereoselective Formation of Cyclic Timers, [Cp*Rh(AMP)]3," Inorg. Chem. 32, 4119 (1993).

D.P. Smith, M.T. Griffin, M.M. Olmstead, M.F. Maestre, and R.H. Fish, "Bioorganic Chemistry. 4. The Bonding Roll of Carbonyl and Amino Functionalities in the Reactions of Guanine Nucleobase Derivatives with ( $\eta^{5}$-Pentamethylcyclopentadienyl)rhodium Complexes in Methano and Aqueous Solutions," Inorg. Chem. 32, 4677 (1993).

\section{Genetic Map of Canis Familiaris}

Principal Investigators: Jasper D. Rine and Elaine A. Ostrander

Funding: \$100,000 (FY 93)

Project No. 92023 $\$ 100,000$ (FY 92)

\section{Project Description}

We recently proposed that a genetic map of the domestic dog can be constructed. Once such a map was in place, long-term-genetic studies on dog behavior, morphology, and diseases would be initiated. Such studies could provide insight into the well-recognized breed-specific differences in appearance, temperament, and behavior that tharacterize the modern dog species. In addition, the biochemical basis for diseases that appear to be sporadic in the general human population, such as lupus and certain types of cancer, could be mapped and studied in the dog where there is a strong genetic component and it is relatively easy to obtain pedigrees with large numbers of affected animals. An ideal set of markers to be used in the assembly of a genetic map for either individual chromosomes or whole genomes would be homogeneously distributed, highly informative, easily utilized, and readily transferred between laboratories. 
Microsatellite repeat sequences, such as $(C A)_{n}$, have many characteristics that satisfy these criteria. This class of repeats is extremely abundant, occurring once every $50-150 \mathrm{~Kb}$ in mammalian genomes.

Microsatellites often exhibit length polymorphisms, and individual markers may have multiple, highly informative alleles. These length polymorphisms are detected by polymerase chain reaction (PCR)-based assays necessitating only nanogram amounts of sample for each genotype. The specificity of each marker comes from the choice of unique sequence primers that flank the microsatellite repeat. PCRbased assays are readily adaptable to automated or semiautomated formats allowing for rapid, large-scale mapping efforts.

The approach entails constructing genomic libraries, which we term marker-selected libraries, that are highly enriched for clones which are apt to contain microsatellite repeats. Such repeats, such as $(C A)_{n}$, have been shown to be ideal for construction of genetic maps because they are homogeneously distributed, highly informative, easily utilized, and often exhibit length polymorphism, with a single marker having several alleles in the population. Constructing marker-selected libraries entails the construction of a small insert primary library, followed by a selection step in which the majority of clones lacking a microsatellite are destroyed. The resultant libraries are highly enriched for clones that are apt to contain a microsatellite and its surrounding sequences. Once these clones have been sequenced, PCR primers are constructed from unique sequences that bracket the repeat to serve as "tags" for distinguishing one microsatellite repeat from another. The products of PCR reactions with the oligonucleotide tag and genomic DNA are analyzed on denaturing sequencing gels to determine which alleles a given individual has a given locus. The level of cosegregation of alleles from different markers in pedigrees of informative individuals provides information about the genetic distance between markers. About 350 evenly spaced markers will be necessary to construct a genetic map that has, on average, $10 \mathrm{cM}$ resolution.

\section{Accomplishments}

First, a large block of simple sequence repeat (SSR) polymorphisms for the dog genome has been isolated and characterized. Screening of primary libraries by conventional hybridization methods as well as by construction of enriched marker-selected libraries led to the isolation of a large number of genomic clones that contained $(C A)_{n}$ repeats. The sequences of 101 clones showed that the size and complexity of $(\mathrm{CA})_{n}$ repeats in the dog genome was similar to that reported for these markers in the human genome. Detailed analysis of a representative subset of these markers revealed that most markers were moderately to highly polymorphic, with Polymorphism

Information Content (PIC) values exceeding 0.70 for $33 \%$ of the markers tested. An association between higher PIC values and markers containing longer $(\mathrm{CA})_{\mathrm{n}}$ repeats was observed in these studies, as previously noted for similar markers in the human genome. A list of primer sequences that tag each characterized marker is provided, and a comprehensive system of nomenclature for the dog genome is suggested.

Second, genetic differentiation, the evolutionary product of mutation, gene flow, genetic drift and selection, is a fundamental quantity of populations that is often difficult to measure precisely. Although studies of the rapidly evolving mitochondrial genome have provided new insights into the structure of closely related populations, the maternal and clonal mode of inheritance of mitochondrial DNA may bias evolutionary reconstructions. Most nuclear loci studies in natural populations evolve slowly and exhibit little polymorphism, consequently a highresolution nuclear perspective on population-level variation has yet to be demonstrated. Microsatellite loci, which are tandem repeats of simple sequences, are hypervariable in length and wide abundant in eukaryotic genomes. Although microsatellite loci have been used extensively in gene mapping and to a limited extent, to deduce paternity and social structure, they have not yet been used to deduce patterns of genetic differentiation among natural populations. We assess variation in 10 microsatellite loci across the geographic range of three species of wolf-like canids to characterize levels of genetic variation, gene flow and relatedness, and to test hypotheses for the origin of the red wolf. Past research on mitochondrial DNA diversity of red wolves suggested the possibility that it may be a form derived from repeated hybridization of coyotes and gray wolves. We provide definitive nuclear and new mitochondrial DNA evidence for this hypothesis based on population samples of living and extinct red wolves.

Third, 93 new dinucleotide repeat polymorphisms specific for the canine genome have been identified and characterized. Screening of both primary canine 
genomic libraries and marker-selected libraries that were enriched for simple sequences repeats led to the isolation of large numbers of clones that contained $(\mathrm{CA})_{n}$ repeats. Over 500 of these clones were sequenced, and $P C R$ primer that bracket the repeat were developed for those that contained ten or more continuous $(\mathrm{CA})_{n}$ units. This led to the production of 93 markers that were polymorphic when tested on a small population of mixed breed dogs. A subset of these markers 23 are considered highly polymorphic and had 5 or more alleles when tested on a panel of unrelated dogs.

\section{Publications}

E.A. Ostrander, G.F. Sprague, and J. Rine, "Identification and Characterization of Dinucleotide Repeat (CA) n Markers for Genetic Mapping in Dog," Genomics 16, 1 (1993).

M.S. Roy, E. Geffen, D. Smith, E. Ostrander, and R.K. Wayne, "Microsatellite Loci as a Population Genetic Tool: A Hybrid Origin of the Red Wolf Confirm," submitted to Trends in Genetics.

E. Ostrander, G.F. Sprague, and J. Rine, "NinetyThree New Sample Sequence Repeat-Based Markers for the Canine Genome," to be submitted to Genomics.

\section{Head Probe for Nuclear Magnetic Resonance Imaging and Spectroscopy at $10 \mathrm{~T}$}

Principal Investigators: Mark S. Roos, Sam T.S. Wong, and Thomas F. Budinger

Funding: $\$ 48,100$

Project No. 93012

\section{Project Description}

The objective of this project is to develop rf coil technology critical to ${ }^{1} \mathrm{H}$ NMR spectroscopic imaging of objects the size of the human head at $10 \mathrm{~T}$. This technology will be a major component in a future generation of NMR spectrometer that will have substantial improvements in sensitivity over current machines. Applications of such instruments include in vivo biomedical research, plant studies related to global climate change, geophysics, and materials science.

Novel volume coils operating at up to $430 \mathrm{MHz}$ have been designed and fabricated and will be tested to determine their suitability for NMR studies. Direct characterization of the probes is not possible because large bore NMR magnets operating at fields of 8 to $10 \mathrm{~T}$ do not yet exist. However, the relevant performance parameters including the signal-to-noise ratios (SNR) for NMR experiments, local and global power deposition, and field homogeneity can be deduced from benchtop measurements. We will measure these parameters in two classes of phantoms: one designed to validate theoretical predictions and the other to duplicate the geometry of the human head. This project will thus provide technology essential for in vivo NMR at $10 \mathrm{~T}$ and experimentally verify predictions for the performance of these instruments.

\section{Accomplishments}

\section{Design and Fabricate a Prototype Head Coil to Operate at $400 \mathrm{MHz}$}

Prototype coils based on the preliminary design have been constructed. It was found difficult to obtain sufficiently high resonance frequencies with this design, and that the inductance of the end rings is undesirable. A capacitively shortened waveguide resonator was studied that has been employed as a body coil operating at $170 \mathrm{MHz}$ for $4 \mathrm{~T}$ whole body MR by Siemens. A prototype head resonator was constructed with two segmented inner conductors on a $30-\mathrm{cm}$ transformer in a 35- $\mathrm{cm}$ cylindrical shield, shown in Fig. 4. The two sides of the probe are

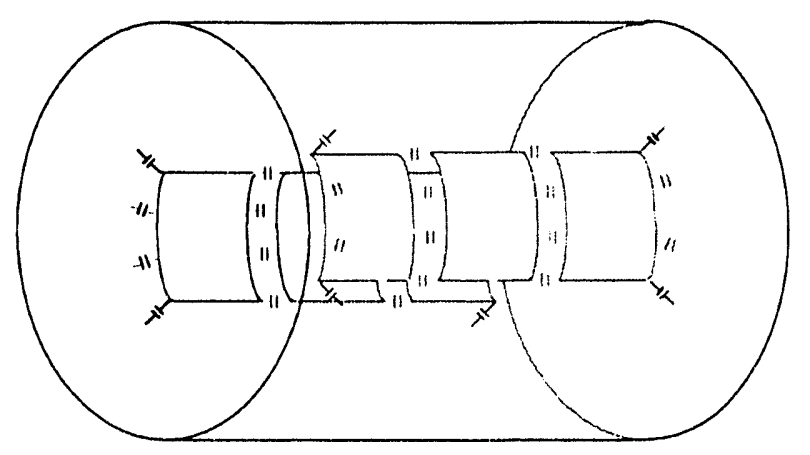

Fig. 4. Diagram of capacitively shortened waveguide resonator. 
connected by a $\lambda / 2$ balun, and driven across the end capacitors. This structure resonates at up to $460 \mathrm{MHz}$. As built, the structure will produce a linearly polarized B field in its interior. The next steps will be an addition of two more segmented inner conductors and construction of a quadrature hybrid and matching circuits for production of a circularly polarized field.

It is desirable to have a mathematical model of the probe that can predict the spectrum of resonances and the associated current distributions. This would aid in selecting component values and improving the drive circuit. We have developed a model consisting of three segments of transmission line connected by capacitive coupling networks. This model has not yet produced accurate predictions of the spectrum of the structure, but it does have similar features. The probable source of error is that the transmission line segments are short, and that interactions among segments and the terminations at the ends of the probe are not described properly. We will continue to investigate whether a model can be constructed without resorting to 3-D numerical methods, which are not available to us.

\section{Construct an Apparatus for Surveying of Fields}

A number of loop detectors have been constructed incorporating zero-bias Schottky barrier diodes (Hewlett Packard HSCH-3486), chip capacitors, and high-impedance plastic leads (Polymer Corp., Reading, Pennsylvania). These devices have been tested using annular resonators up to $400 \mathrm{MHz}$ and have been demonstrated to have a minimal effect on the impedance of the resonator, as well as usable linearity. We are convinced that this approach will work for surveying the fields produced by the heat resonator in air and in conducting phantoms.

The next step in this project is to survey the rf field, measure loading and local heating in cylindrical, spherical, and human head phantoms, and compare with spherical shell model. 


\section{Materials Sciences Division}

\section{Valence-Band Photoemission from Semiconductor Nanocrystals}

Principal Investigator: A. Paul Alivisatos

Funding: $\$ 79,600$

Project No. 93013

\section{Project Description}

The surface of an inorganic nanocrystal plays an important role in determining its structural, thermodynamic, optical, and transport properties. Because of low symmetry and the absence of long-range order on the crystal surface, traditional probes of surface structure are not applicable. Thus more is known about the interior composition and symmetry of nanocrystals than about the surface. Only one previous experiment has been done on II-VI nanocrystal surfaces. This experiment used nuclear magnetic resonance (NMR) to determine bonding geometry and surface coverage of thiophenol on CdS nanocrystals. NMR is probably the most powerful probe available for study of the bonding geometry at the nanocrystal surface. However, it does not immediately provide a route to the full composition of the surface. X-ray photoelectron spectroscopy provides such a probe of composition. Recently, we developed techniques that allow the chemical binding of nanocrystals to $\mathrm{Au}$ and $\mathrm{Al}$ surfaces. The binding molecule situates the nanocrystals $\sim 12 \AA$ from the metal surface, allowing for electron tunneling between the nanocrystals and the surfaces in order to avoid charging. In addition, the samples are supported on a macroscopic surface. This has allowed the exploration of the range of more traditional surface techniques for use on nanocrystals.

\section{Accomplishments}

The first use of a traditional surface science technique, $x$-ray photoelectron spectroscopy (XPS), is reported to explore the surface properties of semiconductor nanocrystals. Crystalline, nearly monodisperse CdSe nanocrystals ranging in radius from 9 to $30 \AA$ were chemically synthesized and covalently bound to $\mathrm{Au}$ and Si surfaces for study. XPS core-level peak positions for $\mathrm{Cd}$ and Se were in agreement with those of bulk CdSe. We have determined that the majority of Se atoms on the surface are unbonded as prepared and that $\mathrm{Cd}$ atoms are bonded to the surface ligand, tri-n-octyl phosphine oxide (TOPO), to the extent that such bonding is sterically allowed, corresponding to total ligand saturation of the nanocrystal surface of from $60 \%$ in the smaller crystals to $30 \%$ in the larger crystals. The saturation coverage is higher than that reached by extrapolation to a homogeneous, uniformly spherical surface, and agrees better with the assumption of the development of surface inhomogeneity, e.g., facets, in the larger nanocrystals. In addition, we have determined that upon exposure of the nanocrystals to air, Se surface sites are oxidized, forming a $\mathrm{SeO}_{2}$ surface film that causes the nanocrystals to degrade over time. The nanocrystal surface can be modified by dissolving the crystals in pyridine. Nearly all of the P ligands are removed in this case, leaving behind primarily unsaturated $\mathrm{Cd}$ and Se surface atoms. In this case, both $\mathrm{Cd}$ and Se will oxidize upon exposure to air.

\section{Publications}

J.E. Bowen-Katari, V.L. Colvin, A.N. Goldstein, A. Tu, and A.P. Alivisatos, "X-ray Photoelectron Spectroscopy of CdSe Nanocrystals with Applications to Studies of the Nanocrystal Surface," to be submitted to J. Chem. Phys.

\section{Electronic Thermalization in Metals and Semiconductors}

Principal Investigator: Jeffrey Bokor

Funding: $\$ 180,900$

Project No. 93014

\section{Project Description}

The goal is to obtain quantitative data on hot electron relaxation mechanisms and rates in a variety of metals and semiconductors. This information can be used to 
optimize electronic device performance in the nanoscale regime and to design laser photochemical processes on metal surfaces.

Time-resolved photoemission spectroscopy will be used as the principal technique for directly measuring laser-excited hot electron energy distributions, and the time evolution of these distributions as they thermalize and equilibrate by various energy loss and momentum exchange processes. In conjunction with this experimental effort, theoretical models capable of describing the results will be developed, and the relevant scattering parameters will be determined.

\section{Accomplishments}

Laser laboratory equipment to be used for these studies has been transported to Berkeley. A new femtosecond laser system design has been completed, which will involve this equipment as well as the addition of several new subsystems. These new subsystems have been ordered. The basic laboratory facility has been completed, and construction of the laser systern is in progress.

A comprehensive theoretical approach to modeling the evolution of nonthermal electron energy distributions produced by femtosecond laser excitation has been developed. This approach is to solve the Boltzmann transport equation with a minimum of approximations. Previous work indicates that the shape of the energy distrib' 1tion changes dramatically on the time scale of interest, so a simple relaxation time approximation approach will not be adequate. It is also known that electron-electron collisions are affecting the distribution on the same time scale as electron-phonon collisions. We are therefore taking the ambitious approach of solving the full Boltzmann equation with both the electron-electron and electronphonon collision kernels included simultaneously. A computer code implementing this has been completed and tested. Good agreement has been found between the calculations and previous experiments. Execution times are only a few hours on a Sparcstation-10.

\section{Publications}

J. Bokor, "Ultrafast Dynamics of Electrons at Surfaces," abstract for the Interdisciplinary Laser Science Conference, Oct. 3-8, 1993.

\section{MOCVD Growth and Characterization of II-VI Compounds Using Nontoxic Single-Molecule Precursors}

Principal Investigators: Edith D. Bourret and John Arnold

Funding: $\$ 75,000$ (FY 93)

$\$ 73,100$ (FY 92)

Project No. 92025

\section{Project Description}

The purpose of this proposal is to develop new nontoxic single-molecule precursors for metal-organic chemical vapor deposition (MOCVD) growth of II-VI compounds. There are two main goals:

(1) To be able to tailor-make specific single-source precursors incorporating any element of choice (for binaries, ternaries, and/or dopants) so that we will be in a position to tailor and control at the molecular level the thin-film properties prior to growth.

(2) To provide replacements for highly toxic conventional precursors. Conventionally, the growth of II-VI compounds by MOCVD involves co-pyrolysis of toxic metal alkyls (such as dimethylzinc) with a volatile highly toxic dialkyl or hydride chalchogenides (such as $\mathrm{H}_{2} \mathrm{Se}$ ). The use of these compounds is heavily regulated and very cosily to industry. There is an urgent need to develop new nontoxic precursors: the environmental and economical impact for the semiconductor industry will be enormous.

The research is conducted in two main areas:

(1) Synthesis and characterization of the novel singlemolecule precursors. This research is conducted through development of new synthetic routes to produce molecular precursors not accessible with previously known techniques and through design of the molecular precursors for specific semiconductor thin films and thin-film properties.

(2) MOCVD growth and characterization of thin films using the new precursors. This research involves study of the pyrolysis reactions and of the kinetics of thinfilm growth, design and fabrication of a specific growth reactor, and characterization of the thin films. 


\section{Accomplishments}

The research results have shown that the proposed single-molecule precursors can indeed be synthesized and are perfectly suited for thin-film growth by MOCVD. A new reactor specifically designed for use of the new precursors has been built, and new sources of funding are needed to pursue the synthesis research and fully explore the use of the new precursors in the newly built reactor for epitaxial growth of II-VI semiconductors.

New $\mathrm{Zn}, \mathrm{Cd}$, and $\mathrm{Hg}$ chalcogenolates that contain the ctel, csel, and sisel ligands were synthesized by procedures similar to those developed in the first year of this program for the $\mathrm{Zn}$ and $\mathrm{Cd}$ sitel complexes. As expected based on similar steric bulk, the ctel, csel, and sisel ligands form complexes with similar physical and spectroscopic properties to their sitel analogues. Thus, they all melt cleanly and may be sublimed at low pressure. The steric bulk of the sitel, sisel, ctel, and csel ligands has made it possible to synthesize volatile complexes of the group 12 metals that pyrolyze cleanly to give deposition of thin films of $\mathrm{ZnTe}, \mathrm{CdTe}$, and $\mathrm{ZnSe}$. These films have been characterized by Rutherford backscattering, to obtain information on their stoichiometry and thickness, and $x$-ray diffraction to identify their crystalline phase. We have demonstrated that new classes of singlemolecule precursors can be used for organometallic vapor phase epitaxy of II-VI compounds.

The most interesting and promising result is the successful deposition of $\mathrm{ZnSe}, \mathrm{ZnTe}$, and $\mathrm{CdTe}$ are very low temperatures (ZnSe on GaAs: $310-345^{\circ} \mathrm{C}$, ZnTe on InAs: $285-310^{\circ} \mathrm{C}$, and CdTe on Si: 240 $345^{\circ} \mathrm{C}$ ). Low-temperature deposition is essential to prevent reactions between the substrate and the film that result in degradation of the heterointerface and of the film electronic and optical properties. The deposition temperature that is obtained, in some cases, is even lower than those obtained using conventional precursors.

These preliminary deposition experiments were limited by the features of the very simple reactor (mainly a thermal gradient tube furnace) that was used. Encouraged by the initial success, the second year was spent designing and building a new reactor specifically for use with the solid precursors. With this reactor we will be able to flow hydrogen gas that is mandatory to properly clean the substrates surface prior to growth of the films. The precursors are oxygen sensitive. A glove box attached to the reactor will be used to load the precursors in controlled atmosphere free of oxygen. We will have the choice of carrier gas: nitrogen or hydrogen. The reactor growth chamber is a vertical chamber equipped with a discursive flange to get a laminar flow of the sublimed precursors onto the substrates. The deposition experiments can be done at atmospheric pressure or at reduced atmosphere. The construction of the reactor is now completed.

\section{Publications}

J. Arnold, J.M. Walker, K.M. Yu, P.J. Bonasia, A.L. Seligson, and E.D. Bourret, "Growth of II-VI Thin Films from Single-Source Precursors Based on Sterically Encumbered Sitel Ligands," J. Crystal Growth 124, 647 (1992).

A.L. Seligson, P.J. Bonasia, J. Arnold, K.M. Yu, J.M. Walker, and E.D. Bourret, "Single-Source Precursors for the Growth of Metal-Chalcogenide Thin Films," Mat. Rec. Soc. Symp. Proc. 282 (1993).

P. Bonasia, A. Seligson, J. Arnold, E. Bourret, and J. Walker, "Volatile Homoleptic Tellurolates and Selenolates of $\mathrm{Zn}, \mathrm{Cd}$ and $\mathrm{Hg}$ and Their Use as SingleSource Precursors to II-VI Semiconducting Thin Films, presented at the 203rd ACS National Matting, San Francisco, April 5-10, 1992.

\section{Optical Investigations of Quantum Well Structures under High Magnetic Fields}

Principal Investigator: Daniel S. Chemla

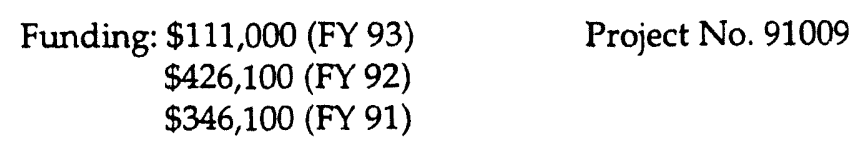

\section{Project Description}

The project was for the investigation of optical properties and ultrafast dynamics of elementary excitations of semiconductors and their heterostructures under very high magnetic fields.

Modern crystal growth techniques are now able to produce very high-quality semiconductor thin films with thickness controlled at atomic-layer level. These novel artificial materials have had a tremendous 
impact on the fundamental sciences of solid state and have already produced numerous applications. Attempts to produce lower-dimensionality nanostructures (quasi-1D or 2D) have been plagued by the difficulties of fabricating samples without defects and of avoiding size fluctuations.

By applying large magnetic fields on semiconductors, the electronic states are further confined to $0 D$ or 1D. Thus a convenient way to avoid the difficulties associated with sample fabrication, and yet investigate the physics of low dimensionality and potential applications, is to apply high magnetic fields to semiconductors to further the confinement to OD and 1D. The approach to this project exploited recent advances in time-resolved laser spectroscopy and split-coil superconducting magnets to investigate the physical properties of elementary excitation in lowdimensionality regime.

\section{Accomplishments}

The optical laboratory for femtosecond time-resolved spectroscopy has been completed. The experimental set up for nonlinear optical measurements inside the cryostat-magnet assembly has been built and tested. New experiments at high magnetic fields have started. Three ongoing projects are already producing very exciting results:

(1) Investigation of instantaneous frequency dynamics of coherent emission from magnetoexcitons in gallium arsenide. Experiments were performed at $1.6 \mathrm{~K}$ and $\mathrm{B}=6 \mathrm{~T}$ using $100 \mathrm{fs}$ laser pulses. The ultrafast nonlinear optical magnetoexciton response was measured by simultaneously resolving spectrally and temporally coherent wave mixing emission (see Fig. 1). Quantum interference affecting the amplitude and the phase of the emitted light has been observed.

(2) Utrafast nonlinear optical response of Fano resonances in gallium arsenide under high magnetic field. It was found that the coupling of high order 1D-

magnetoexcitons with 1D-continuum of free particle states results in Fano resonances. Using femtosecond spectroscopy, we study for the first time, the dynamics of the coherent emission from Fano resonances and compare it to that from an isolated Lorentzian line. We find that despite the very narrow frequency lineshape, the coupling to the continuum induces an almost instantaneous nonlinear optical response.

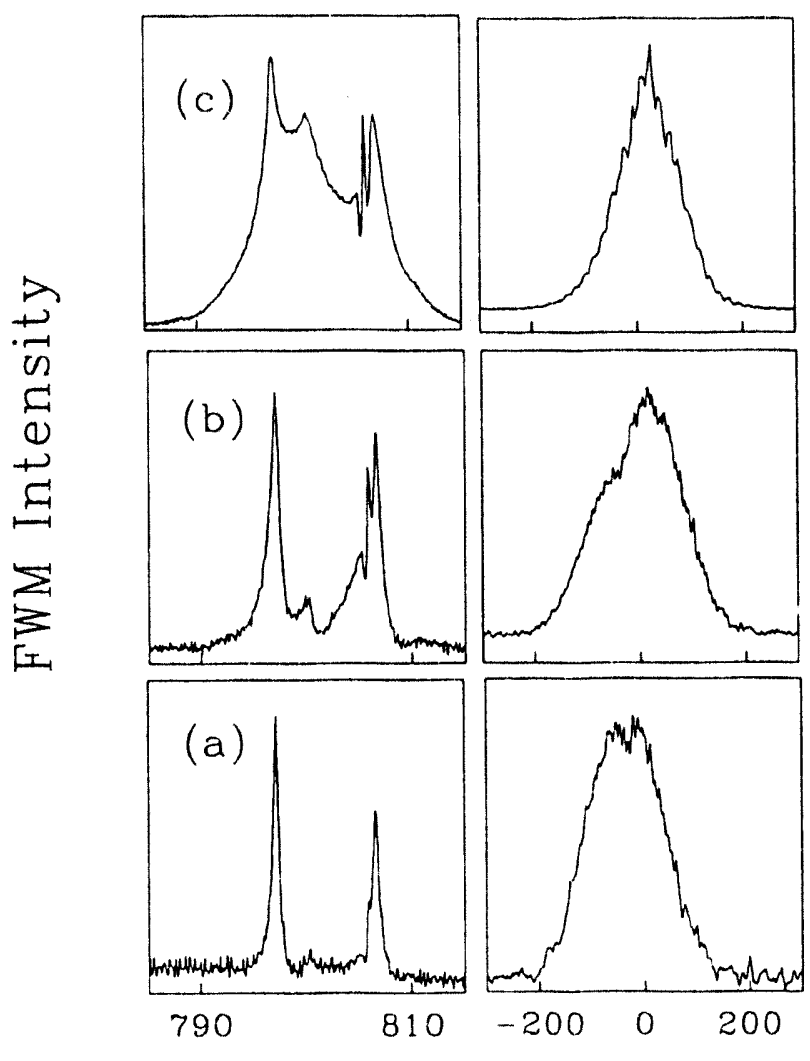

Wavelength ( $\mathrm{nm}$ ) T'ime Delay

Fig. 1. Time dependence (right column) and spectral content (left column) of the coherent emission of gallium arsenide at $6 T$, for three excitation densities by ultrashort light pulses. The potver spectra reveal density dependent quantum interferences between the magnetoexcitons.

(3) Temporal and spectral investigation of multi-Landau level quantum beats in $\mathrm{GaAs}$. By resolving temporally and spectrally transient wave-mixing generated by a ultrafast spectrally broad laser pulse, we observe multi-Landau level quantum beating in $\mathrm{GaAs}$ at moderate, $B=6 \mathrm{~T}$, magnetic field. We observe for the first time nonperiodic beats that originate from complex density dependent quantum interferences between coupled states in the material (see Fig. 2).

In conclusion, the preliminary results already obtained indicate that the control of the dimensionality by application of high magnetic fields provides the ideal laboratory for studying both the effects of quantum confinement on the dephasing properties of and interactions between elementary excitations in semiconductors. 


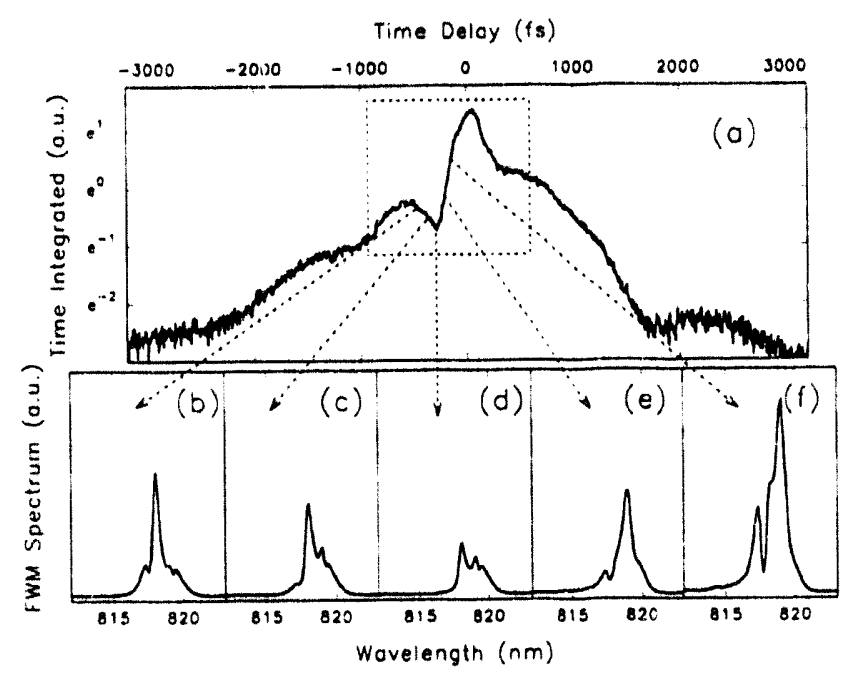

Fig. 2. Upper trace, time dependence of logarithm of the total intensity of the coherent emission. The lower traces present the spectral content of the emission at five time delay between $-560 \mathrm{fs}$ and $-160 \mathrm{fs}$. The changes in the power spectra show an extremely fast rate of modulation of the instantaneous frequency.

\section{Publications}

M.-A. Mycek, U. Siegner, and D.S. Chemla, "Instantaneous Frequency Dynamics of Coherent Emission from Magnetoexcitor $\dot{s}$ in Gallium Arsenide," submitted to the 1994 International Quantum Electronics Conference, Anaheim, CA.

U. Siegner, M.-A. Mycek, and D.S. Chemla, "Utrafast Nonlinear Optical Response of Fano Resonances in Gallium Arsenide Under High Magnetic Field," submitted to the 1994 International Quantum Electronics Conference, Anaheim, CA.

U. Siegner, M.-A. Mycek, and D.S. Chemla, "Temporal and Spectral Investigation $n$. Multiple Landau Level Quantum Beats in GaAs," sutmitted to the 1994 International Quantum Electronics Conference, Anaheim, CA.
Electron-Beam Lithographic Fabrication of Submicron Junctions for Macroscopic Quantum Tunneling, Coulomb Blockade and High- $\mathrm{T}_{c}$ SQUIDS

Principal Investigator: John Clarke

Funding: $\$ 119,800$

Project No. 93015

\section{Project Description}

The first goal of the project is to apply expertise in electron beam lithography gained on an elderly, borrowed ETEC Autoscan scanning electron microscope to a new JEOL JSM-6400 scanning electron microscope. This electron-beam writing machine is then to be used to pattern submicron tunnel junctions for two different experiments. The first is a study of resonant tunneling in submicron Josephson junctions. Such junctions are expected to make transitions from the zero voltage state to the voltage state at bias currents far below the thermodynamic critical current because of macroscopic quantum tunneling (MQT) between quantum states. Observations of this novel phenomenon would shed new light on MQT between discrete states. The second experiment is an investigation of the Coulomb blockade of submicron normal metal-insulator-normal metal junctions with self-capacitance $C \leqq 10^{-15} \mathrm{~F}$, cooled to millikelvin temperatures. The junctions interconnect an array of quantum dots each with a capacitance $\mathrm{C}_{g}$ to $a$ groundplane. These arrays are predicted to exhibit novel dynamical critical phenomena such as metastability and hysteresis.

\section{Accomplishments}

During the first three quarters of the year we continued to use the ETEC microscope; the JEOL microscope was finally installed at the Center for XRay Optics in July 1993. Because of the delayed arrival of the new machine, all of the Josephson 
junctions were made on the ETEC machine, while the arrays have been made on the JEOL.

The Josephson junctions were fabricated using a double-layer resist in which a narrow bridge is suspended above the substrate. The first aluminum film was evaporated at an angle of about $45^{\circ}$ to the substrate, and then oxidized. The second aluminum film was evaporated after tilting the substrate through about $90^{\circ}$, so that the second film overlapped the first in a narrow region to form a small area tunnel junction. Four high-resistance leads each consisting of a narrow, thin $\mathrm{NiCr}$ film were connected to each junction to permit current-voltage characteristics to be measured. In the current series of devices, two such junctions are connected in parallel to form a dc Superconducting QUantum Interference Device in which the critical current can be adjusted by means of a magnetic field. At millikelvin temperatures these devices switch randomly between the zero voltage and the voltage states, and a detailed study of the mechanism involved is currently underway.

The arrays of junctions are also fabricated from aluminum films, using the technique described above. In the experiment, the films are driven into the normal state by a magnetic field of about 0.5 tesla. To achieve a known, controllable capacitance $C_{g}$ between the quantum dots and a groundplane, we use a degenerately doped silicon substrate. A thermal oxide grown on the surface of the silicon electrically isolates the array from the substrate, which serves as the groundplane. The procedure involves eleven processing steps and produces oxide thicknesses within $1.5 \%$ of the design goal. To date, the JEOL electron beam writer has been used to produce $10 \times 10$ test arrays. An electron micrograph of such an array is shown in Fig. 3, and an image at higher magnification showing a single quantum dot and the associated four junctions is shown in Fig. 4. For topological reasons, the diamond-shaped arrangement of islands in the array leads to a square array of junctions. As can be seen, junctions with lateral dimensions approximately $40 \mathrm{~nm}$ by $60 \mathrm{~nm}$ have been fabricated in this way. These dimensions are close to the state-of-the art of junctions made with these techniques. The small arrays shown in Fig. 3 will be used to ascertain that such parameters as the tunneling resistance and capacitance to ground $C_{g}$ are correct prior to making the large (up to 70 by 70 ) arrays required for the quantum transport measurements. It is expected that experiments on these large arrays will begin early in 1994.

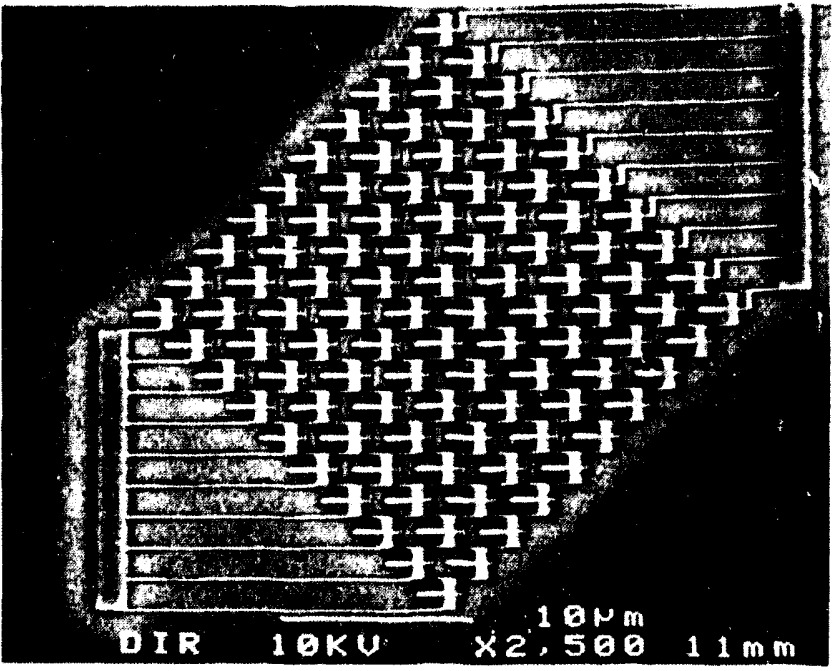

Fig. 3. Electron micrograph of $10 \times 10$ test array consisting of $\mathrm{NiCr}$ on a silicon substrate. The array was fabricated using shadow evaporation techniques; $\mathrm{NiCr}$ was used to make the array visible in the electron microscope. Individual islands are clearly visible as well as bus bars (on two sides of the array) for application of bias voltage. The unit cell size for the array is $2.3 \mu \mathrm{m}$ by $2.3 \mu \mathrm{m}$. (XBB 9311-7633A)

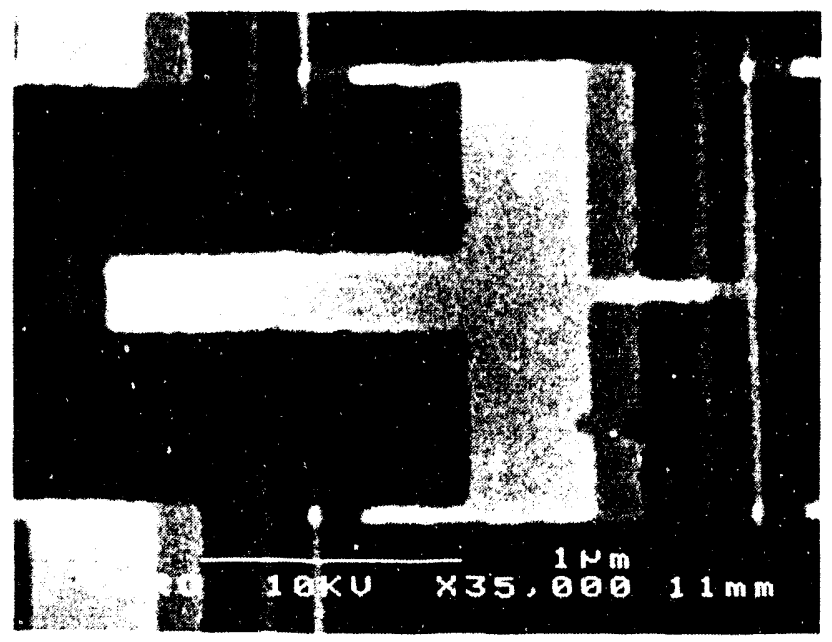

Fig. 4. Electron micrograph of a single island from the array in Fig. 3. Four junctions with dimensions approximately $40 \mathrm{~nm}$ by $60 \mathrm{~nm}$ are clearly visible at the intersections of the narrow vertical and horizontal lines. (XBB 9311-7633B) 


\section{Novel Surface and Interface Studies Using Photoelectron Spectroscopy Combined with Scanning Tunneling Microscopy}

Principal Investigator: Charles S. Fadley

$\begin{aligned} \text { Funding: } \$ 96,000 \text { (FY 93) } & \text { Project No. } 91011 \\ \$ 129,000 \text { (FY 92) } & \\ \$ 193,900 \text { (FY 91) } & \end{aligned}$

\section{Project Description}

New methods are being developed for the study of surfaces, interfaces, and nanostructures and are being applied to novel surface morphologies that have been synthesized as part of the research. A principal focus is on experimental characterization techniques utilizing synchrotron radiation. This work also involves combining synchrotron radiation measurements with independent characterizations by complementary techniques such as scanning tunneling microscopy (STM).

The principal experimental methods used are photoelectron spectroscopy a.ld photoelectron diffraction, which together can provide detailed information on both the atomic geometries of surface species in different chemical or magnetic states and the valence electronic structure that binds them together. Photoelectron diffraction is also being extended into a holographic analysis, with this development promising to produce accurate three-dimensional images of near-surface atomic and magnetic structures for the first time.

An additional important component of the work is the theoretical interpretation of photoelectron diffraction patterns and the development of more efficient and accurate methods of extracting accurate structural information from them. This involves unique computer codes for calculating diffraction patterns, as well as for holographically inverting these patterns to produce real-space images.

\section{Accomplishments}

\section{Surface Structure Determinations from Photoelectron} Holography

The intriguing prospect of being able to use photoelectron diffraction patterns above surfaces to determine surface atomic structures directly by holographic imaging methods is a very recent idea.
Our group is still one of relatively few in the world working to assess the feasibility of this approach. Experimental studies of both the bulk semiconductors $\mathrm{Si}$ and $\mathrm{Ge}$ and a simple adsorbate system $(\mathrm{S} / \mathrm{Ni}$ ) show that holographic images do indeed contain features associated with near-neighbor atoms. Although bulk images are found to be more affected by aberrations, for the adsorbate test case of $\mathrm{S} / \mathrm{Ni}$, the most accurate nearest-neighbor images from experimental data to date have been produced. We have also explored theoretically the various ways in which such holographic data can be obtained including scannedangle and scanned-energy approaches, and studied the possibility of using spin-polarized photoelectron holography to image magnetic moments for the case of a cubic lattice of $\mathrm{MnO}$.

\section{Application of Ultrahigh Vacuum STM to Metal-} Semiconductor and Metal-Metal Interactions

In preparation for combining STM with photoelectron diffraction and holography at the ALS, we have continued to use a custom-built ultrahigh vacuum STM. This system provides for in situ transfer of specimens from the STM to stations for either metal deposition, ion bombardment, or LEED/Auger characterization. Our first study with this system was of the interaction of $\mathrm{Sn}$ with $\mathrm{Ge}(111)$. High-quality STM images with atomic resolution were obtained for several types of structures including the clean $c(2 \times 8)$ surface, room-temperature-deposited Sn atoms and microclusters, and an annealed $\mathrm{Sn} / \mathrm{Ge}$ structure that is very similar to the well-known $(7 \times 7)$ reconstruction on $\mathrm{Si}(111)$. These images furthermore contain several new structural elements not observed before. A second and ongoing study involves the epitaxial growth of Gd on W(110), a system that has been studied in some detail for its unusual surface magnetic properties, but never before with STM. Our STM images have revealed thie precise growth modes of epitaxial Gd.

\section{Combination of Photoelectron Diffraction and STM in a Single System}

We have completed a custom-built chamber and special sample manipulator that have been added to an existing photoelectron spectrometer so as to permit performing for the first time photoelectron diffraction and STM measurements on the same sample. This system is presently being used to study the growth of iron oxide on $\mathrm{Pt}(111)$. We are at present obtaining a complete set of photoelectron diffraction data for determining the internal structure of this oxide that should permit much better understanding the growth of this and other epitaxial magnetic oxides. 


\section{Advanced Photoelectron Spectrometer/Diffractometer for the ALS}

Considerable progress has also been made during the current year on a unique next-generation and station that will be used at the ALS. This new system will provide ultrahigh resolutions in both energy $(\Delta E / E=$ $10,000)$ and emission angles $\left( \pm 1.0^{\circ}\right.$ or better). It will also permit detecting electrons at the extremely high rates that are expected due to the high brightriess of the ALS, as well as measuring spin-resolved electron spectra in studies of magnetic systems.

\section{Other Surface Structure Studies Based upon Photoelectron Diffraction}

- The structure of silver on Si(111). In a study of the $(\sqrt{3} \times \sqrt{3})$ overlayer of $\mathrm{Ag}$ on $\mathrm{Si}(111)$, both scannedangle and scanned-energy photoelectron diffraction data were obtained, and both the atomic structure and the Ag coverage better determined.

- High-temperature surface phase transition on $\mathrm{Ge}(111)$. This surface disordering transition occurs $150^{\circ}$ below the melting point. Our results include holographic analyses below and above the transition temperature, and they indicate that the transition involves a layer about 2 atoms in thickness.

- Theoretical simulation of photoelectron and Auger electron diffraction results. This work is centered on a general-purpose program that we have written for doing both single-scattering and fullyconverged multiple-scattering calculations of such diffraction patterns, based on a new separable Green's function method. During the current year, the speed of this code has been increased by another order of magnitude and has been modified so as to be able to excite the photoelectrons with either linearly-polarized or circularly-polarized radiation.

- Spin-polarized photoelectron diffraction. The addition of spin resolution to the photoelectron diffraction measurement has also been studied theoretically in both a single-scattering and multiple-scattering framework. The holographic analysis of this type of data shows promise for directly imaging magnetic moments.

- Effects of multiple scattering on effective sampling depths in photoelectron spectroscopy and diffraction. In another theoretical study, we have shown via numerical simulations that the strong multiple elastic scattering effects involved in photoelectron diffraction can lead to marked change in the effective sampling depth below the surface as a function of emission direction. This result is important for the quantitative analysis of photoelectron intensities so as to determine surface compositions.

\section{Publications}

T.T. Tran, S. Thevuthasan, Y.J. Kim, D.J. Friedman, A.P. Kaduwela, G.S. Herman and C.S. Fadley, "Photoelectron Diffraction and Photoelectron Holography Study of a Ge(111) High-Temperature Surface Phase Transition," Surf. Sci. 281, 270 (1993).

G.S. Herman, E.L. Bullock, M. Yamada, A.P. Kaduwela, D.J. Friedman, S. Thevuthasan, Y.J. Kim, T.T. Tran, C.S. Fadley, Th. Linder, D.E. Ricken, A.W. Robinson, and A.M. Bradshaw, "A Scanned-Angle and Scanned-Energy Photoelectron Diffraction Study of $(\sqrt{3} \times \sqrt{3})$ R3O\# Ag on Si(111)," Surf. Sci. 284, 23 (1993).

K. Higashiyama, S.K. Lewis, R.X. Ynzunza, E.D. Tober, Y.J. Kim, and C.S. Fadley, "Asymmetry of Adatoms on $\mathrm{Ge}(111)$ Observed by Scanning Tunneling

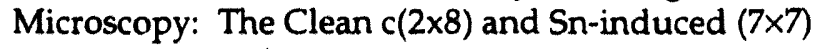
Structures," Surf. Sci. 291, 47 (1993).

S. Thevuthasan, R.X. Ynzunza, E.D. Tober, A.P. Kaduwela, M.A. Van Hove, and C.S. Fadley, "HighEnergy Photoelectron Holography for an Adsorbate Test System: c( $2 \times 2) \mathrm{S}$ on Ni(001)," Phys. Rev. Lett. 70, 595 (1993).

A.P. Kaduwela, M.A. Van Hove, and C.S. Fadley, "Comment on Adsorbate Structures from Photoelectron Diffraction: Holographic Reconstruction or Real-Space Triangulation?," Phys. Rev. Lett. 71, 299 (1993).

A.P. Kaduwela, Z. Wang, S. Thevuthasan, M.A. Van Hove, and C.S. Fadley, "Imaging Short-Range Magnetic Order by Spin-Polarized Photoelectron Holography," submitted to Phys. Rev. Lett.

P.M. Len, S. Thevuthasan, C.S. Fadley, A.P. Kaduwela, and M.A. Van Hove, "Holographic Atomic Imaging from Scanned-Angle and Scanned-Energy Photoelectron Diffraction Data," in preparation.

E.D. Tober, R.X. Ynzunza, C. Westphal, and C.S. Fadley, "Gadolinium Thin Film and Cluster Growth on W(110) as Studied by Scanning Tunneling Microscopy," in preparation. 
R.X. Ynzunza, R. Couch, S. Ruebush, S. Chaniberlain, S. Thevuthasan, A.P. Kaduwela, M.A. Van Hove, and C.S. Fadley, "Effects of Multiple Scattering on Effective Sampling Depths in X-ray Photoelectron Spectroscopy and Diffraction," in preparation.

C.S. Fadley, "Diffraction and Holography of Photoelectrons and Fluorescent X-rays," invited review in Mat. Res. Soc. Symp. Proc. 307, 261 (1993).

C.S. Fadley, "Diffraction and Holography with Photoelectrons and Auger Electrons: Some New Directions," invited review to appear in Surface Science Reports, December 1993.

C.S. Fadley, "Photoelectron Diffraction and Holography: Present Status and Future Prospects," invited review to appear in the Proceedings of the Fifth International Conference on Electron Spectroscopy, Kiev, Ukraine, August 1993 (Elsevier Publishing).

\section{C.S. Fadley, "Photoelectron Diffraction and} Holography: Some New Directions," invited review to appear in the Proceedings of the Fourth International Conference on the Structure of Surfaces, Shanghai, China, August 1993 (World Publishing).

\section{Isotope Heterostructures Selectively Doped by Neutron Transmutation}

Principal Investigators: Eugene E. Haller and Wladyslaw Walukiewicz

Funding: $\$ 45,400$

Project No. 93016

\section{Project Description}

The sy, thesis and processing of semiconductor multilayer sıructures with extremely long minority carrier lifetimes required for high-efficiency photovoltaicenergy conversion was proposed. These structures will also allow the study of dopant-induced intermixing at very high dopant concentrations. Isotopically enriched and chemically pure ${ }^{70} \mathrm{Ge}$ and ${ }^{74} \mathrm{Ge}$ will be used to grow an undoped isotope superlattice by molecular beam epitaxy (MBE). Since host atom diffusion is slower than dopant diffusion, this approach will lead to the most abrupt isotope interfaces. Doping will be achieved by the neutron transmutation process at the Missouri Research Reactor facility. The transmutation doping will produce an npnp superlattice. Photogenerated electrons and holes will be spatially separated by the internal electric fields and can be collected along the $n$ and $p$ layers. The spatial separation will result in reduced electron-hole recombination and thus also in enhanced minority carrier diffusion lengths.

\section{Accomplishments}

Rapid progress towards the proposed goals has been made. High purity, single crystals of isotopically pure ${ }^{70} \mathrm{Ge}$ and ${ }^{74} \mathrm{Ge}$ have been grown successfully. The crystals have been used as substrates for growth of a heterointerface between two different isotopes. Four different isotope heterostructures have been grown in the laboratories of $\mathrm{G}$. Abstreiter at the Technical University in Munich. Two of them are $565 \AA$ thick layers of ${ }^{70} \mathrm{Ge}$ and ${ }^{74} \mathrm{Ge}$ grown on a natural $\mathrm{Ge}$ substrates: one is a $565 \AA$ thick layer of ${ }^{70} \mathrm{Ge}_{0.574} \mathrm{Ge}_{0.5}$ alloy, and the other is a heterostructure consisting of two subsequently grown $1000 \AA$ thick layers of ${ }^{74} \mathrm{Ge}$ and ${ }^{70} \mathrm{Ge}$. Prior to neutron transmutation doping, the structural and electronic properties will be studied in order to assess the quality and chemical purity of the structures.

During preparation of the isotope single crystals, a few crystals were doped with oxygen. In its bond centered position oxygen forms an infrared active center in Ge. Because of the large number of different mass combinations of the two neighboring germanium atoms, the oxygen vibrational spectrum is very complicated in natural Ge [Fig. 5(a)]. A spectacular simplification of this spectrum is achieved through isotope enrichment. In collaboration with A. Ramdas, Purdue University, we have recorded high-resolution spectra with ${ }^{\times} \mathrm{Ge}: \mathrm{O}(\mathrm{x}=70,73,74,76)$ crystals. As an example, the ${ }^{74} \mathrm{Ge}: \mathrm{O}$ spectrum is shown in Fig. 5(b). These high-resolution spectra allow the first complete and unambiguous assignment of all the features in the natural Ge:O spectrum. The above results clearly demonstrate the excellent quality of the single isotope crystals that are being used as substrates for the preparation of isotope heterostructures.

Publications

E.E. Haller, "Semiconductor Isotope Engineering," Solid State Phenomena 32, 11 (1993), Proc. GADEST '93, Klingmühle, Germany, Oct. 9-14, 1993; LBL-34279. 


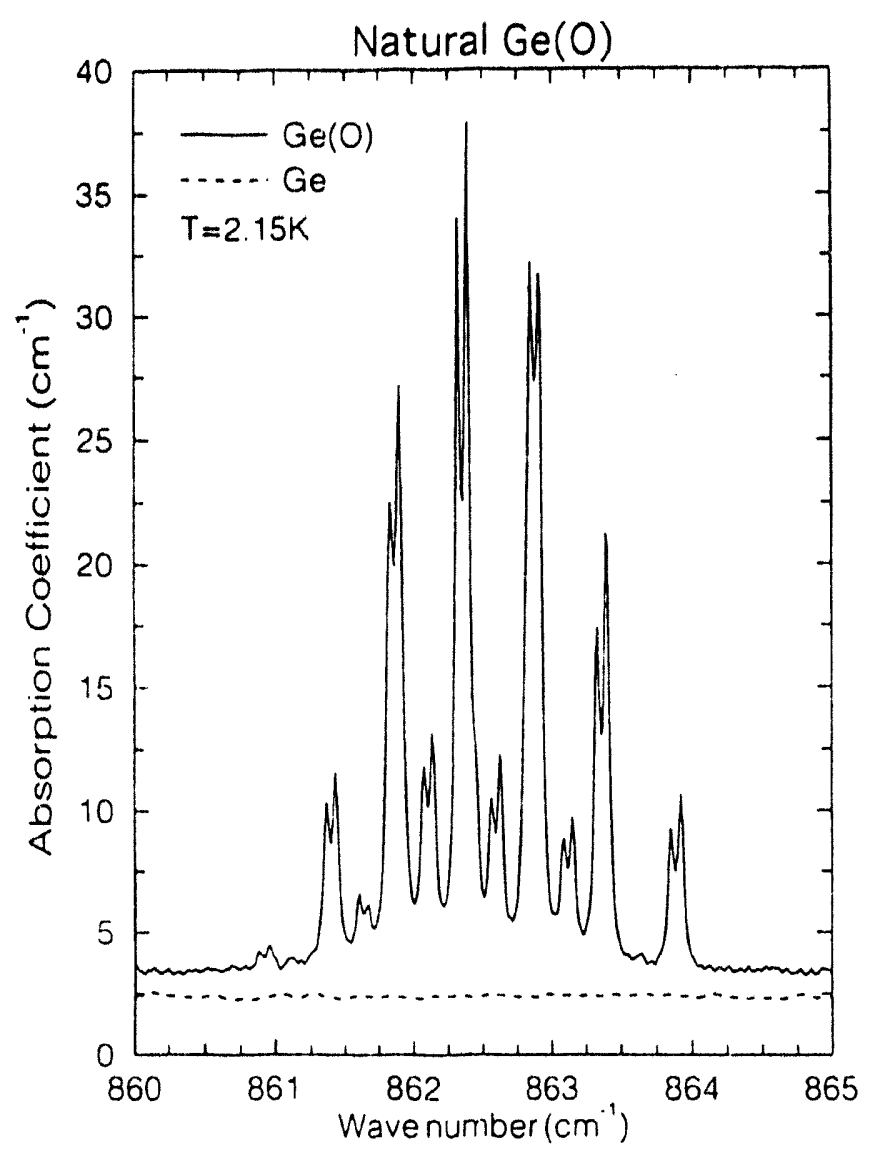

(a)

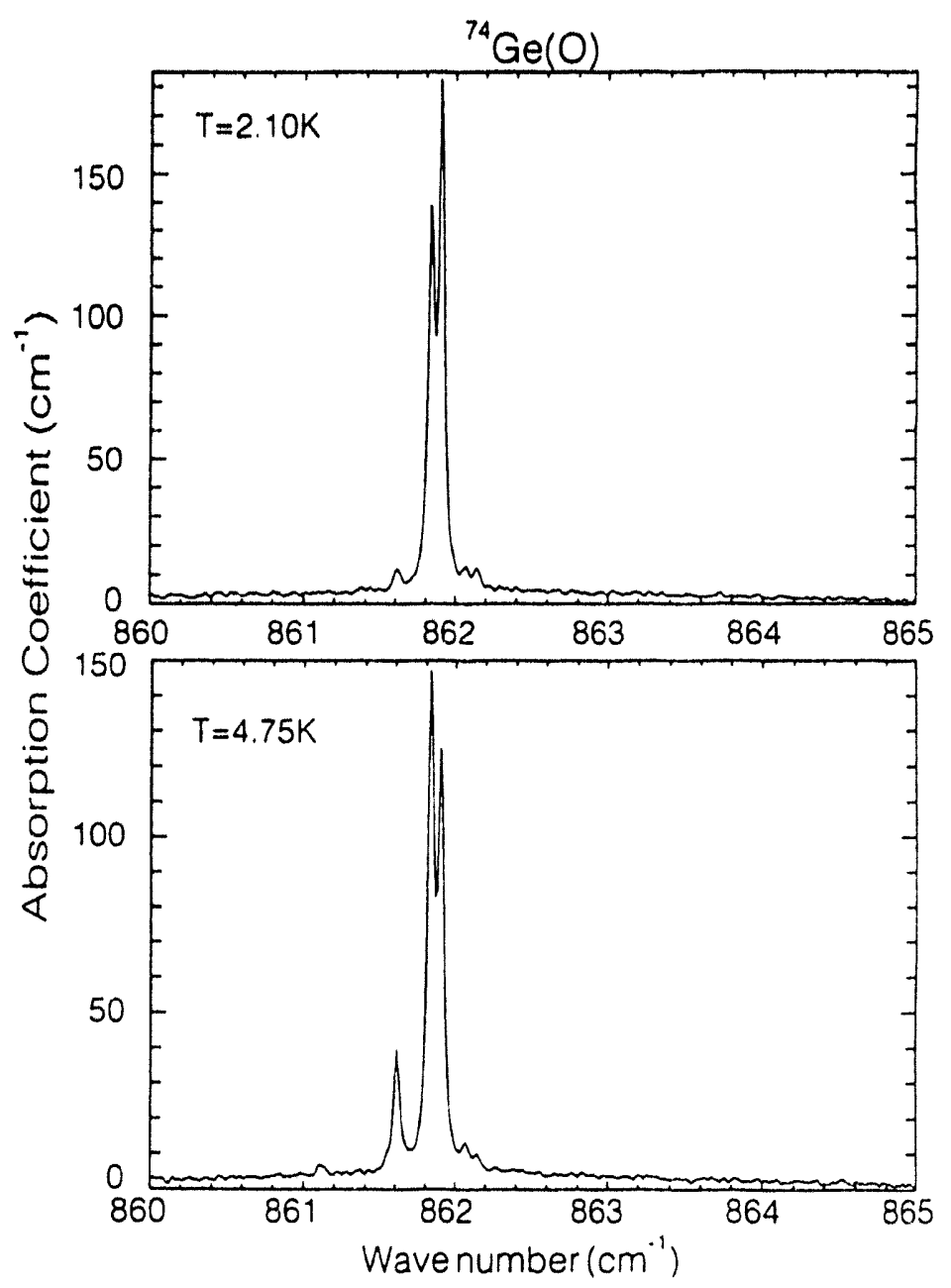

(b)

Fig. 5. Far infrared absorption spec.ra of (a) pure and oxygen doped natural Ge and (b) oxygen doped ${ }^{74} \mathrm{Ge}$ at two temperatures.

H.D. Fuchs, K.M. Itoh, and E.E. Haller, "Isotopically Controlled Germanium: A New Medium for the Study of Carrier Scattering by Neutral Impurities," submitted to Phil. Mag. (M. Cardona Festschrift); LBL34848.

E.E. Haller, "Physics with Chemically and Isotopically Pure Semiconductors," Acta Physica Polonic, A84, 395 (1993).

J. Spitzer, T. Ruf, M. Cardona, W. Dondl, R. Schorer, G. Abstreiter, and E.E. Haller, "Raman Scattering by Optical Phonons in Isotopic ${ }^{70}(\mathrm{Ge})_{n}{ }^{74}(\mathrm{Ge})_{n}$ Superlattices," submitted to Phys. Rev. Lett.

\section{Electron Transport in Nanostructures}

Principal Investigator: Paul L. McEuen

Funding: $\$ 71,400$ (FY 93) $\$ 114,800$ (1992)

Project No. 92028

\section{Project Description}

This project is to study ballistic and phase-coherent electron transport in semiconductor nanostructures at low temperatures and very high-magnetic fields. Ultrafast measurement techniques will also be 
developed to investigate transient transport phenomena in nanostructures. An electron beam lithography system will be used to define structures with features as small as $10 \mathrm{~nm}$. A dilution refrigerator/superconducting magnet system is used to investigate these structures at low temperatures $(\mathrm{T}=10 \mathrm{mK})$ and high-magnetic fields $(B=14 \mathrm{~T})$. In addition, ultrafast laser pulses and photoconductors are used to study the dynamics of transport on a picosecond time scale.

Nanostructures are artificially patterned systems whose characteristic dimensions lie midway between the macroscopic and atomic worlds. While their sizes and shapes are under the control of the experimenter, their properties are nevertheless regulated by basic quantum effects. This remarkable situation opens up many interesting avenues for research. For example, nanostructures may be used as a laboratory for studying the physics of quantum transport. In addition, they may be used to construct entirely new materials with novel electronic properties. Initially, we concentrated on the fabrication and measurement of electrons confined within semiconductor heterostructures. The heterostructures are provided through external collaborations, patterned using electron beam lithography facilities to be developed at LBL, and measured in a low-temperature/highmagnetic field system.

\section{Accomplishments}

A number of tasks have been completed and scientific results obtained during the previous year. The lowtemperature/high-magnetic field measurement system has been made fully operational, and, in collaboration with the Center for X-Ray Optics, a new electron beam lithography system has been commissioned. We are currently using these facilities to fabricate and measure a variety of nanostructures, particularly GaAs quantum point contacts (QPCs). These QPCs are lithographically defined constrictions in a two-dimensional electron gas, and initial measurements on these structures were described in last year's final report.

Ultrafast measurements of these QPCs are underway in collaboration with the another LBL group.

Evidence for photon-assisted transport was obtained, and the temperature and magnetic field dependence of the observed effects are currently being studied. In a second set of experiments on QPCs, some exciting but unanticipated results were obtained in high- magnetic fields. Using QPCs as a source of spinpolarized electrons, techniques were developed to create and probe nuclear spin polarization on a submicron scale. Scattering of spin-polarized electrons emerging from a QPC pumps the nuclear spin system, polarizing the nuclei in the vicinity of the QPC. The fraction of the electrons that have spin-flip scattered can be measured using a second QPC, and this can be used to infer the local nuclear polarization and electron spin-nuclear spin coupling.

Unambiguous evidence for the nuclear spin detection is shown in Fig. 6. The current through a constriction at $B=4.7 \mathrm{~T}$ is measured as a function of an applied radio-frequency $(\mathrm{r} f$ ) field. When the applied $\mathrm{rf}$ coincides with the known NMR lines of the Ga or As host atoms, a dramatic change in the current is observed. These experiments clearly demonstrate we can probe nuclear polarization on a submicron scale. This opens up a variety of new possibilities for research. First, we can study questions of nuclear spin diffusion and electron/nuclear spin interactions in a size regime previously inaccessible. Secondly, the properties of the material can be probed through local electrically detected NMR, and perhaps even perform magnetic resonance imaging with submicron resolution.

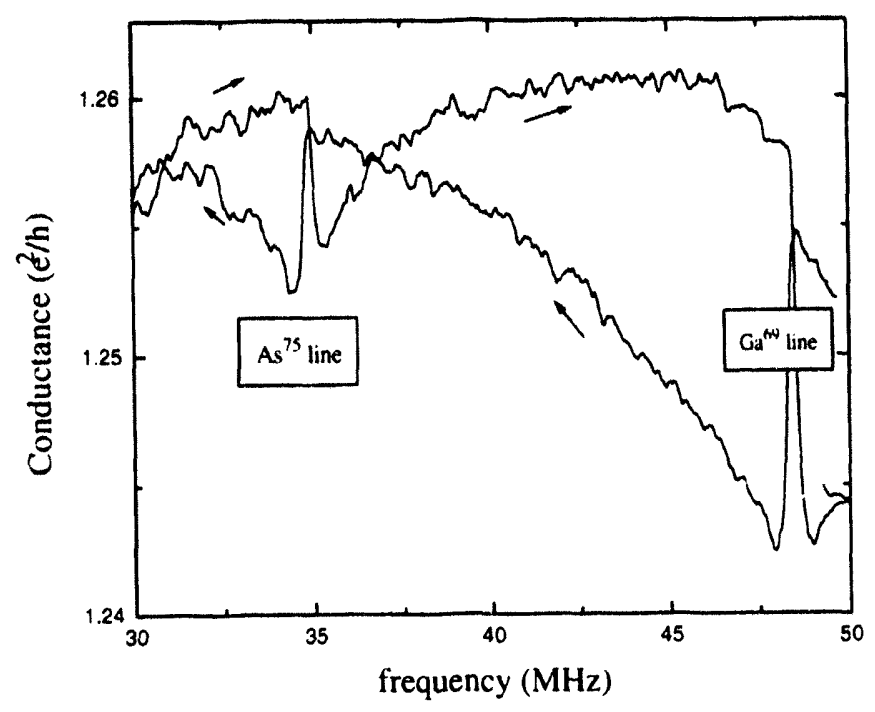

Fig. 6. Local NMR using a QPC. The conductance of a quantum point contact at $B=4.7 \mathrm{~T}$ is measured vs the frequency of an applied of field. Changes are observed at the NMR lines of the GaAs. The arrows indicate the sweep direction. 


\section{Publications}

S. Jahuar, C. Karadi, K.R. Wald, P.L. McEuen, and J. Orenstein, "Response of a GaAs Quantum Point Contact to Microwave Frequencies," abstract submitted to the March Meeting of the American Physical Society.

K.R. Wald, L. P. Kouwenhoven, and P.L. McEuen, "Local Dynamic Nuclear Polarization Using Quantum Point Contacts," to be submitted to Phys. Rev. Lett.

Near-Field Scanning Optical Microscopy/Spectroscopy of LowDimensionality Materials at 50-nm Resolution

Principal Investigators: D. Frank Ogletree and Shimon Weiss

Funding: $\$ 48,000$

Project No. 93017

\section{Project Description}

Novel low-dimensionality materials are being synthesized at LBL. The near-field scanning optical microscope (NSOM) is a new tool that combines the power of optical imaging and spectroscopy with spatial resolution exceeding $1 / 10$ wavelength. The NSOM and related spectroscopic techniques have many potential applications to low-dimensionality materials under development at LBL. Two specific applications are:

(1) chemical synthesis and manipulation of semiconducting nanoclusters, and

(2) synthesis of Langmuir-Blodgett molecular films with di-acetylene groups in the hydrocarbon chains.

The NSOM technique offers many of the advantages of conventional optical microscopy with sub- wavelength or "super-resolution." In common with other scanned probe microscopies such as scanning tunneling microscopy (STM) and atomic force microscopy (ATM), the spatial resolution is determined by the probe geometry, and imaging is accomplished by mechanical translation of the probe in the vicinity of the sample surface.

Optical measurements with sub-wavelength resolution are made using a probe consisting of a single-mode optical fiber drawn down to an end diameter of $<100 \mathrm{~nm}$. Images are made by positioning this probe within $\sim 50 \mathrm{~nm}$ of a surface and scanning the probe as in STM or AFM imaging. High contrast images can be obtained by detecting photoluminescence or fluorescence with this probe from specimens excited by an external light source.

\section{Accomplishments}

Methods for fabricating probes for near-field optical microscopy and spectroscopy have been developed. Single mode optical fibers can be pulled to submicron diameters and metallized.

A microscope for near-field optical microscopy/ spectroscopy has been developed. This instrument operates on the principle of shear-force feedback for imaging. The NSOM fiber probe is osciliated perpendicular to its axis by $\sim 5 \mathrm{~nm}$. This oscillation is monitored by measuring the diftracted light of a diode laser focused on the side of the fiber. As the probe comes within $\sim 10 \mathrm{~nm}$ of the surface, the oscillation is attenuated. This attenuation is used to maintain a constant probe-surface separation during data acquisition independent of optical signals.

The general purpose NSOM microscope can be operated in transmission mode for transparent samples or reflection mode for opaque samples. Samples can be translated by millimeter distances under imaging conditions to find regions of interest. Optical signals can be measured in both photon counting and analog modes.

Reports at the Second International Conference on Near Field Optics (October 93) show that the NSOM technique is capable of detecting single fluorescent molecules and also of performing spectroscopic measurements on semiconductor nanoclusters. 


\section{Quasiparticle Dynamics of High- $T_{c}$ Superconductors: Probing the Symmetry of the Pair Wavefunction}

Principal Investigator: Joseph Orenstein

Funding: \$161,100

Project No. 93018

\section{Project Description}

Many of the most interesting and important phenomena in condensed matter physics are associated with energy scales in the terahertz $\left(10^{12}\right)$ regime of the electromagnetic spectrum. Coherent terahertz spectroscopy, the generation and detection of subpicosecond electrical pulses, promises to open this exciting regime for study. A unique capability for coherent terahertz spestroscopy has been developed at LBL during the previous year. We are currently applying this capability to study the following topics: Quasiparticle dynamics in high- $T_{c}$ superconductors and dynamics of electron propagation in nanostructures.

Devising techniques for the generation and detection of sub-picosecond pulses was the starting point for this research. These pulses have been used to study the systems listed above. Two main approaches have been adopted: linear spectroscopy as a probe of quasiparticle dynamics in high- $\mathrm{T}_{\mathrm{C}}$ superconductors, and correlation spectroscopy to study the dynamics of electrons in nanostructures.

The first approach, linear spectroscopy, is a powerful probe of the electrodynamics of high- $T_{c}$ cuprate-based superconductors. It provides the ability to measure both the real and imaginary parts of the conductivity (or resistivity) of a material without Kramers-Kronig analysis. This is particularly useful in superconducting materials where the response changes from reail to imaginary near $T_{c}$.

\section{Accomplishments}

Recently a great deal of attention has focused on understanding the electrodynamics of cuprate superconductors when placed in a large, steady magnetic field. During the past year a time-domain spectrometer has been designed and implemented that operates in conjunction with a superconducting magnet in order to measure the electrodynamical response of materials in high fields. Using the spectrorneter/magnet system the resistivity of YBCO thin films was measured as a function of frequency from 100 to $800 \mathrm{GHz}$ in fields up to 6 Tesla. In a magnetic field there will be diagonal and off-diagonal components of the resistivity. The off-diagonal component, $\rho_{x y}$, corresponds to the Hall resistivity. To determine both components, we measured the transmission of the terahertz beam through YBCO thin films placed between parallel and crossed wiregrid polarizers ( $t_{x x}$ and $t_{x y}$, respectively). The temperature dependence of the real and imaginary parts of the off-diagonal resistivity, $\rho_{x y}$ at $350 \mathrm{GHz}$, as determined from $t_{x y}$, is shown in Fig. 7.

Similar to dc measurements, we find that $\rho_{x y}$ at high frequency varies roughly as $1 / T$ above $T_{c}$ (not shown) and changes sign just below $T_{c}$. As the temperature is lowered further, other researchers have found that $\rho_{x y}$ at zero frequency becomes unmeasurably small. However, our high-frequency data reveal a remarkable temperature dependence: $\operatorname{Re}\left\{\rho_{x y}(\omega)\right\}$ changes sign again, reaching a positive maximum by $40 \mathrm{~K}$ and dropping to zero at $10 \mathrm{~K}$. The peak is not expected for vortex motion. We believe it is due to the cyclotron motion of quasiparticles in the superconducting state, observed for the first time.

In relation to nanostructures, extensive studies of electronic transport in the quantum regime have led to a deep understanding of the near-zero frequency conductance of mesoscopic structures. The Landauer formula, relating the conductance to the electron

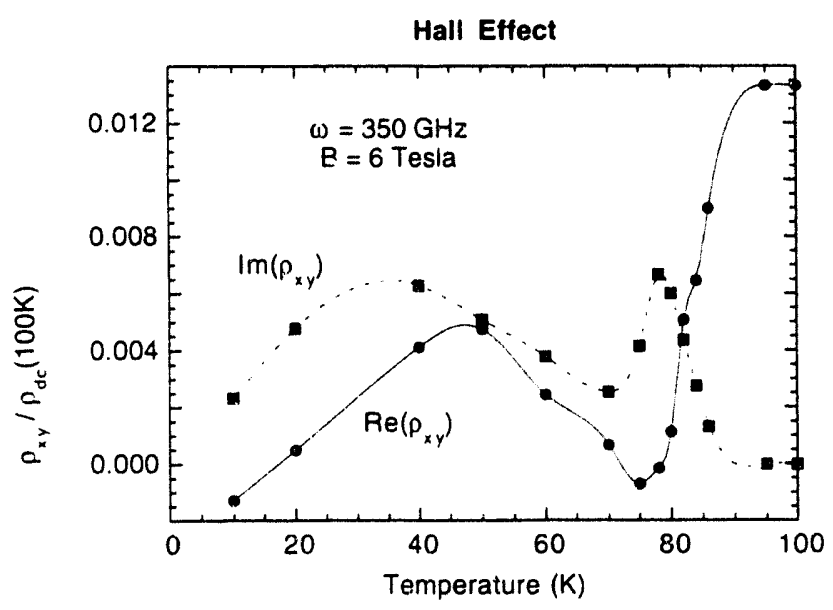

Fig. 7. Real and imaginary parts of the Hall effect at 350 $\mathrm{GHz}$ resulting from the cyclotron motion of quasiparticles in the superconducting state of $\mathrm{YBa}_{2} \mathrm{Cu}_{3} \mathrm{O}_{7}$. The peak near $40 \mathrm{~K}$ is caused by the competition between increasing quasiparticle lifetime and reduced density as the temperature is lowered. 
scattering matrix, provides a unifying viewpoint for understanding such seemingly diverse phenomena as conductance plateaux in the quantum Hall effect and transport through narrow constrictions in a twodimensional electron gas. In contrast, the understanding of high-frequency conductance is much less well developed, owing largely to the comparative lack of experimental results. An experimental study has begun of the high-frequency response of the simplest of mesoscopic structures, the quantum point contact (QPC). We believe that our results offer evidence for photon-assisted transport across a $\mathrm{QPC}$ and provide a foundation for further studies.

The main part of Fig. 8 shows typical curves of the enhanced dc current through the barrier, $\Delta \mathrm{I}$, as a func-

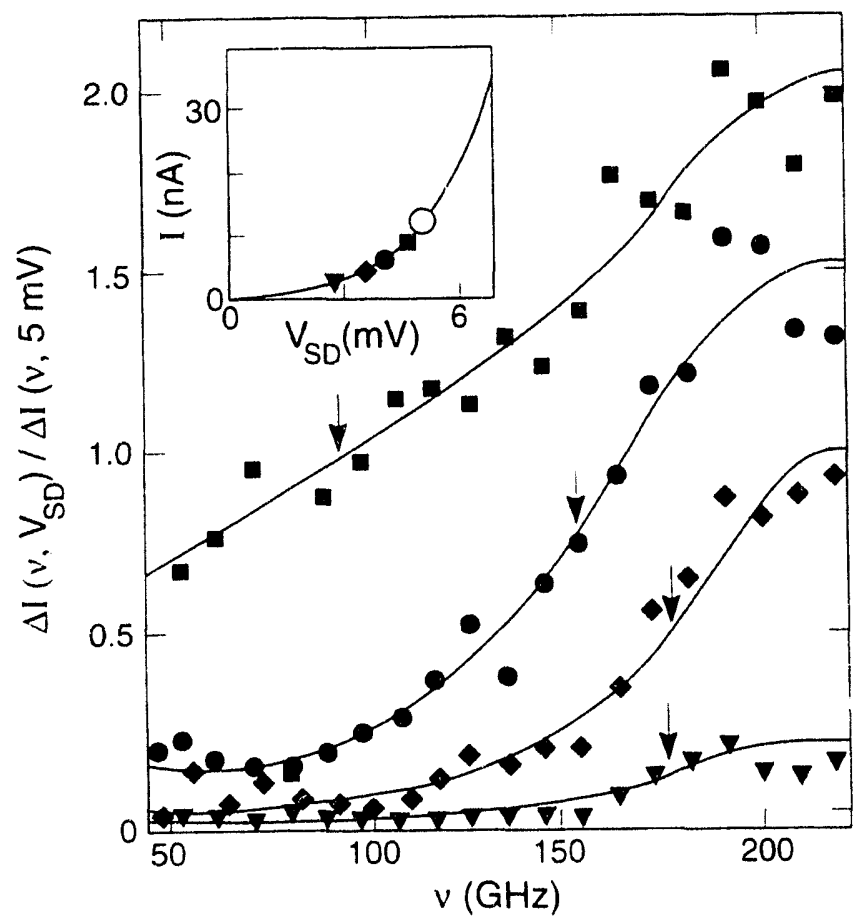

Fig. 8. The magnitude of photon-assisted-transport across a QPC, as function of frequency, for several values of the potential drop across the barrier. The characteristic frequency for the onset of photon-assisted-transport (indicated by the arrows) shifts to lower frequency as increasing bias voltage lowers the barrier. The potential drop for each curve and the corresponding dc current through the barrier are shown in the inset. tion of excitation frequency for several values of $\mathrm{V}_{\text {sd }}$, the voltage across the barrier. The dramatic changes in the spectra as a function of $V_{\text {sd }}$ suggest that the enhanced conductance is due to photon-assistedtransport over the barrier. Consistent with this interpretation, the spectra shift systematically to higher frequency as $\mathrm{V}_{\text {sd }}$ decreases and the height of the barrier grows. In the future we will use our twopulse correlation technique to determine the complex nonlinear admittance of nanoscale structures in the terahertz frequency regime, in which the effects of quantum confinement and Coulomb interaction combine to determine the dynamics.

\section{Publications}

S. Verghese, C. Karadi, C.A. Mears, J. Orenstein, P.L. Richards, and A.T. Barfknecht, "Measured Response of $\mathrm{Nb} / \mathrm{Al}_{2} \mathrm{O}_{3} / \mathrm{Nb}$ Tunnel Junctions to Picosecond Voltage Pulses," presented at Applied Superconductivity Conference, September, 1992, Chicago, IL.

C. Karadi, S. Verghese, C. A. Mears, J. Orenstein, P.L. Richards, A.T. Barfknecht, "Response of $\mathrm{Nb} / \mathrm{Al} 2 \mathrm{O} 3 / \mathrm{Nb}$ Tunnel Junction to Picosecond Electrical Pulses," J. Shah and U. Mishra (eds.), OSA Proceedings on Ultrafast Electronics and Optoelectronics 14 (1993), p. 231.

S. Verghese, C. Karadi, C.A. Mears, J. Orenstein, P.L. Richards, and A.T. Barfknecht, "Response of $\mathrm{Nb} / \mathrm{Al} 2 \mathrm{O} 3 / \mathrm{Nb}$ Tunnel Junctions to Picosecond Voltage Pulses," Bulletin of the Am. Phys. Soc., Transactions, Part A, 38(7), 1624 (1993).

S. Verghese, C. Karadi, C.A. Mears, J. Orenstein, P.L. Richards, and A.T. Barfknecht, "Picosecor d Response of the Quasiparticle Current in Superconducting Tunnel Junctions," Proceedings of the XXth International Conference on Low Temperature Physics, August 1993, edited by R. J. Donnelly, Eugene, Oregon.

S. Verghese, C. Karadi, C.A. Mears, J. Orenstein, P.L. Richards, and A.T. Barfknecht, "Broad-Hand Response of the Quasiparticle Current in a Superconducting Tunnel Junction," Appl. Phys. Lett., in press. 


\section{A Femtosecond Multi-Wavelength Source for Visible/Infrared Spectroscopy}

Principal Investigator: Gregg D. Sucha

Funding: $\$ 118,100$

Project No. 93019

\section{Project Description}

The main objective of this research is the development and use of a multi-wavelength, femtosecond laser source for spectroscopic studies of a variety of materials systems. This source will make it possible, for the first time (with $100 \mathrm{fsec}$ resolution), to investigate electronic-vibronic dynamics of molecules and interband-intraband dynamics of solids. In addition, we study the generation of ultrashort pulses in additive pulse modelocked (APM) lasers, in particular, the nonlinear dynamics of the modelocking process.

We use an amplified, femtosecond, color-center laser system at $1.5 \mu \mathrm{m}$ to generate continuum in the infrared. This continuum can be used to probe vibronic states in molecules and solids. Simultaneously, 3rd and/or 5th harmonic generation can be used to provide green or violet pump pulses for excitation of electronic transitions in these molecules and solids.

\section{Accomplishments}

In the past year, the performance of the amplified $\mathrm{NaCl}$ laser system has been greatly enhanced by two major modifications. First, we have installed a more powerful pump laser that allows either higher pulse energies at the original $1 \mathrm{kHz}$ repetition rate, or the previous power levels $(20 \mu \mathrm{J})$ at repetition rates up to $5 \mathrm{kHz}$. Second, we have installed an improved lithium-niobate pockels cell (a pulse-picker) that has acoustic damping. This allows pulse switchout at repetition rates up to $10 \mathrm{kHz}$ with improved contrast ratio over the old pockels cell. Currently, this system generates continuum at $5 \mathrm{kHz}$ reliably enough to perform pump-probe measurements.

The generation of powerful pulses in the visible is necessary for the direct excitation of electronic transitions in molecules. Starting from a wavelength of $1.5 \mu \mathrm{m}$, this requires third harmonic generation (THG) to produce green light $(\lambda=500 \mathrm{~nm})$. We have succeeded in generating significant amounts of third harmonic in calcite. Starting with about $2 \mu \mathrm{J}$ of infrared light, we obtain about $200 \mu \mathrm{J}$ of green light.
This corresponds to a peak power of $1 \mathrm{MW}$ in the green. This process is also accompanied by continuum generation, which may or may not be deleterious to the THG process. By frequency doubling the $1.5 \mu \mathrm{m}$ pulses, we have also been able to generate up to $3 \mu \mathrm{J}$ of light at $750 \mathrm{~nm}$. This light is sufficiently powerful to generate continuum in quartz, thus allowing simultaneous generation of broadband continuum around $750 \mathrm{~nm}$ and $1.5 \mu \mathrm{m}$. In the process of developing this source, we have also discovered a number of previously unknown aspects of laser behavior. We have found that our laser system exhibits period-doubling and quasi-chaotic behavior under certain conditions. Experimental and theoretical studies of this behavior have helped us to shed new light on the dynamics of APM laser systems.

We have used the improved setup and the improved continuum generation to perform femtosecond measurements of carrier relaxation and the nonlinear optical response of InGaAs quantum wells as well as bulk InGaAs, as shown in Fig. 9. These differential absorption spectroscopy (DAS) measurements are the first of their kind at this wavelength. This represents a significant advance in ultrafast studies in this technologically important material. It also clearly demonstrates the usability of the amplified $\mathrm{NaCl}$ system.
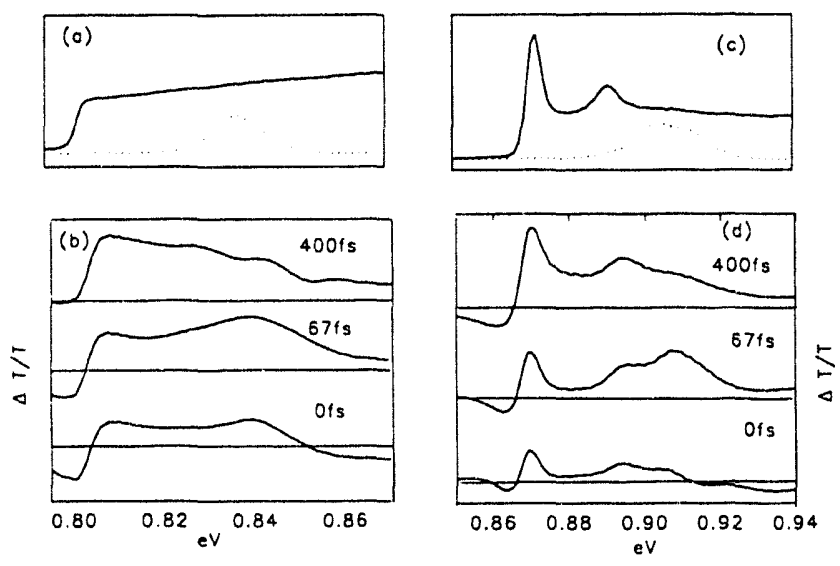

Fig. 9. Absorption and differential absorption spectra (DAS) of $6000 \AA$ and $100 \AA$ InGaAs samples. These spectra demonstrate the high-quality pump-probe data available with the continuum system, and show important differences in many body interactions between two and threedimensional semiconductors. (a) Absorption spectrum (solid) and pump spectrum (dotted) of $6000 \AA$ sample. (b) $D A S$ of $6000 \AA$ sample. (c) Absorption spectrum and DAS of $100 \AA$ sample. (d) DAS of $100 \AA$ sample. 


\section{Publications}

G. Sucha, S.R. Bolton, S. Weiss, and D.S. Chemla, "Period-Doubling and Quasiperiodicity in Additive Pulse Modelocked Lasers," Optics Lett., in press.

S.R. Bolton, G. Sucha, D.S. Chemla, D. L. Sivco, and A.Y. Cho, "Dimensionality Dependence of Semiconductor Nonlinear Optical Response," QELS, Baltimore, MD, May 1993.

G. Sucha, S.R. Bolton, and D.S. Chemla, "Nonlinear Dynamics of Additive Pulse Mode-locked Lasers: period doubling and chaos," CLEO, Baltimore, MD, May 1993.

G. Sucha, S.R. Bolton, D. Botkin, D.S. Chemla, D. L. Sivco, and A.Y. Cho, "Dimensionality Dependence of Semiconductor Nonlinear Optical Response," APS March Meeting, Seattle, WA, March 1993.

S.R. Bolton, G. Sucha, D. Botkin, D.S. Chemla, D.L. Sivco, and A.Y. Cho, "Carrier Dynamics in InGaAs Quantum Wells: Dependence on dimensionality," APS March Meeting, Seattle, WA, March 1993.

\section{Time-Resolved Studies of VUV, XUV, and Soft-X-Ray Photoinduced Chemistry of Surfaces}

Principal Investigator: Harry W. K. Tom

Funding: $\$ 123,800$

Project No. 93020

\section{Project Description}

The purpose of this research is to study mechanisms for surface chemical reactions induced by high-energy photon photoexcitation. Previous interest in this field has been limited to ion desorption (Photon-Stimulated Ion Desorption, PSID). These new investigations extend to more complex chemical reactions. The mechanisms of photon-stimulated chemistry are particularly relevant to semiconductor XUV and soft$x$-ray photolithography. A better understanding of the reaction mechanisms may open new ways of manipulating surface photochemistry and improving semiconductor processing.

The laser-based techniques will be used to timeresolve the surface chemical reactions on the 30$50 \mathrm{fsec}$ time-scale. Measurement of the reaction time after a 30-50 fsec VUV or soft-x-ray initiating pulse will allow a unique way to distinguish between direct photochemical processes that occur in less than $100 \mathrm{fsec}$, indirect secondary electronic processes that occur during the first 1 psec, and lattice thermal processes that continue for several psec. The pump VUV or soft-x-ray pulse will be generated by highharmonic generation in a gas. A second time-delayed probe pulse will measure the evolution of the chemical reaction on the surface by surface secondharmonic generation or UV photoemission.

This program specifically develops the capability to manipulate XUV and soft-x-ray beams (focusing, beam-splitting, beam-combining, time-delaying, and beam overlapping) required in time-resolved measurements at synchrotrons such as the Advanced Light Source (ALS) at LBL. The optics will be developed in close collaboration with $\mathrm{D}$. Attwood and J. Underwood at LBL's Center for X-Ray Optics.

\section{Accomplishments}

The laboratory has been renovated to support highpower laser experiments. An amplified laser system is being built to generate $35 \mathrm{fs}$ pulses at $800 \mathrm{~nm}$ at energies up to $75 \mathrm{~mJ}$ per pulse. So far, a Kerr-lens modelocked Ti:sapphire oscillator has been built producing $30 \mathrm{fs}$ pulses at $800 \mathrm{~nm}$. Two multipass amplifiers and a grating stretcher and compressor have been designed for maintaining a $35 \mathrm{fs}$ final amplified pulse width. An ultrahigh vacuum surface science chamber with standard surface probes and an electron time-of-flight tube for time-resolved photoemission measurements have been setup, and construction begun of a second subchamber for generating the high harmonics in a rare gas jet. 


\section{Picometer-Scale Structure of Interfaces from Synchrotron Radiation}

Principal Investigators: Michel A. Van Hove, Charles S. Fadley, and Philip N. Ross

Funding: \$133,000 (FY 93) Project No. 92031 $\$ 137,000$ (FY 92)

\section{Project Description}

Many physical, electronic, optical, magnetic, chemical, tribological, mechanical, and other properties of modern materials depend intimately on their atomicscale structure. Modern materials increasingly rely on the special properties of selected interfaces between chemically or structurally different substances. This project has developed novel methods that can determine the structure of solid/vacuum and solid/solid interfaces on the sub-angstrom scale, using synchrotron radiation from LBL's Advanced Light Source (ALS).

We have used the techniques of photoelectron diffraction, including forward focusing of electrons and electron holography, both of which are novel forms of photoelectron diffraction with great future potential. These techniques have recently undergone fundamental development to deliver sub-angstrom accuracy. Forward focusing and electron holography, in particular, show great promises, especially in conjunction with synchrotron radiation. Emphasis has been on the development of these techniques for studying solid/vacuum and solid/solid interfaces.

\section{Accomplishments}

Major achievements have been obtained using the techniques of photoelectron diffraction, photoelectron holography, $x$-ray fluorescence holography, and circular dichroism in photoelectron angular distributions.

The state-of-the-art Rehr-Albers computer codes for modeling photoelectron data were vastly accelerated, while also being made portable to workstations. A "linear photoelectron diffraction" perturbation method has been developed and implemented to accelerate the important process of surface structural determination. Various holographic transformations that produce 3-dimensional atomic images from measured data and theoretical simulations were successfully implemented and tested in several cases,
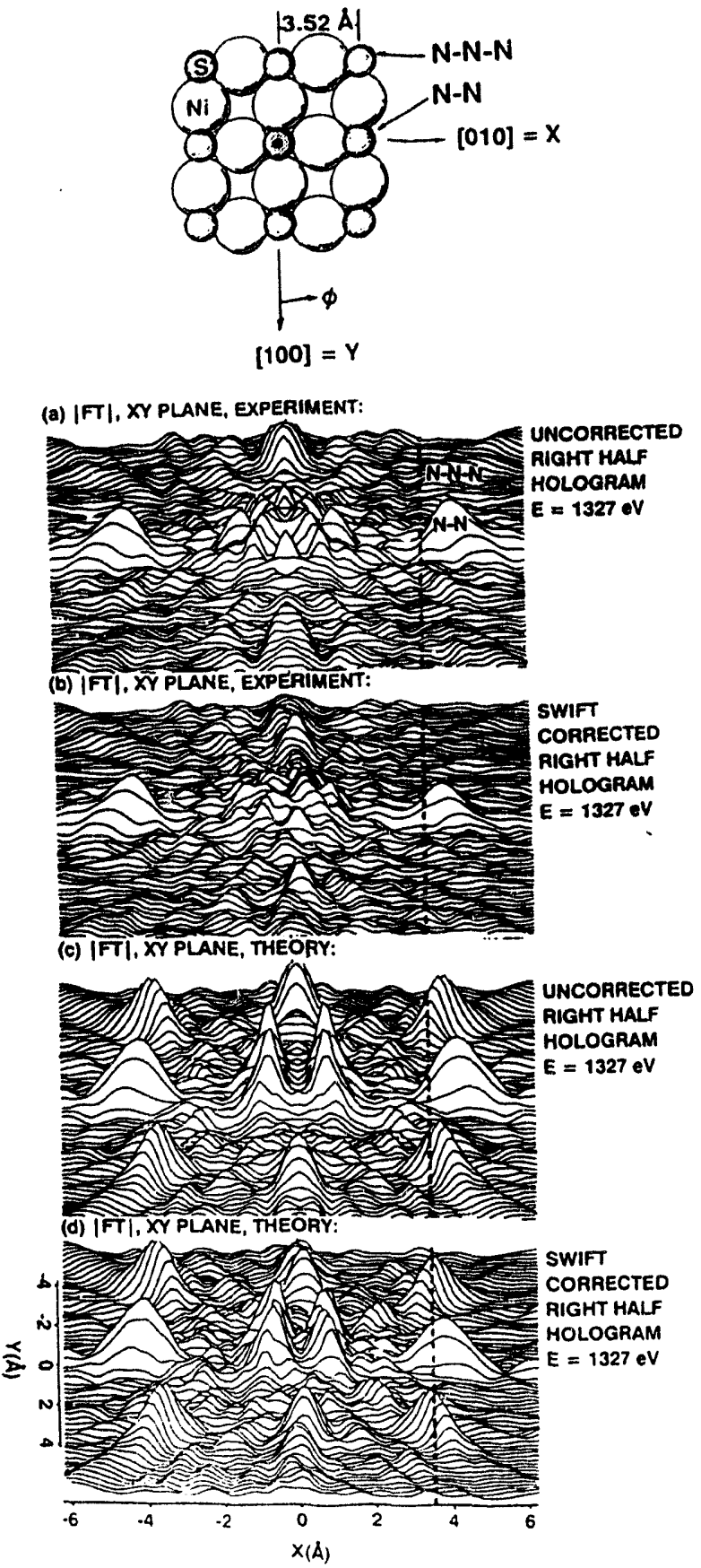

Fig. 10. Experimental $(a, b)$ and theoretical $(c, d)$ holographic images of the $S$ atoms in the test case $c(2 \times 2) S / N i(001)$ whose geometry is shown in the upper panel. The S emitter is at the center of the cluster. These images were obtained from a single-energy $(1327 \mathrm{eV})$ hologram measured over a selected region minimizing nonideal scattering effects; also, only the right half of the hologram was analyzed to optimize the imaging of atoms with positive $x$. Images are shown without any correction for scattered-wave effects ( $a$ and $c$ ) and with correction for scattering phase shifts ("SWIFT") (b and d). 
including sulfur atoms adsorbed on the $\mathrm{Ni}(001)$ surface (Fig. 10): such test cases have helped us identify the critical issues for extending the method to other interfaces. The $\mathrm{S} / \mathrm{Ni}$ results have provided the most accurate single-energy holographic images obtained to date, with excellent agreement between experiment and theory.

Parallel theoretical analyses have also been conducted for simulated spin-polarized data on surfaces of $\mathrm{Mn}^{2+}$ lattices and chains of $\mathrm{Mn}^{2+}$ and $\mathrm{O}^{2-}$. The goal is to image short-range magnetic order, leading to what can be termed spin-polarized photoelectron holography (SPPH). The work established the possibility to determine both the positions of magnetic scatterers and the orientations of the magnetic moments relative to that of the emitter. The results are very encouraging and suggest that three-dimensional images of magnetic moments should be derivable from data that will be obtained with next-generation synchrotron radiation sources such as the ALS.

We have furthermore theoretically simulated $x$-ray fluorescence holography (XFH). This approach suggests the possibility of in principle using photon diffraction patterns to obtain even better images of atoms than with electrons. Indeed, due to the much weaker and more isotropic nature of $\mathrm{x}$-ray scattering, the image peaks in $\mathrm{XFH}$ are found to be within $\approx 0.1 \AA$ of their true atomic positions, with no satellite features, unlike the usual electron case. Theoretical interpretation of circular dichroism in photoelectron angular distributions (CDAD) from core levels has also been undertaken and shown to promise additional insight into surface electronic and structural properties.

\section{Publications}

A.P. Kaduwela, M.A. Van Hove, and C.S. Fadley, "Linear Photoelectron Diffraction: Application of a Rapid Approximation for Surface Structural Studies," Surf. Sci. Lett., in press.

S. Thevuthasan, G.S. Herman, A.P. Kaduwela, T.T. Tran, Y.J. Kim, R.S. Saiki, C.S. Fadley, and M.A. Van Hove, "Electron Emission Holography of Small Clusters and Surfaces," J. Vac. Sci. Technol. A10, 2261 (1992).
A.P. Kaduwela, S. Thevuthasan, Y.J. Kim, M.A. Van Hove, and C.S. Fadley, "Theoretical Modeling of Electron Emission Holography: How Accurately Can We Expect to Image Atoms?," Bull. Am. Phys. Soc. 37, 694 (1992).

C.S. Fadley, S. Thevuthasan, R.X. Ynzunza, E. Tober, R.S. Saiki, Y.J. Kim, A.P. Kaduwela, and M.A. Van Hove, "A Test of Photoelectron Holography for the System c( $2 \times 2) \mathrm{S}$ on $\mathrm{Ni}(100), "$ Bull. Am. Phys. Soc. 37, 694 (1992).

S. Thevuthasan, R.X. Ynzunza, E.D. Tober, C.S. Fadley, A.P. Kaduwela, and M.A. Van Hove, "HighEnergy Photoelectron Holography for an Adsorbate Test System: c(2×2)S on Ni(001)," Phys. Rev. Lett. 70, 595 (1993).

A.P. Kaduwela, Z. Wang, S. Thevuthasan, M.A. Van Hove, and C.S. Fadley, "Imaging Short-Range Magnetic Order by Spin-Polarized Photoelectron Holography," submitted to Phys. Rev.

C.S. Fadley, "Diffraction and Holography with Photoelectrons and Auger Electrons: Some New Directions," Surface Science Rep. 19, 231 (1993).

M.A. Van Hove, "Low-Energy Electron Diffraction and Electron Holography: Experiment and Theory," in Equilibrium Structure and Properties of Surfaces and Interfaces, Eds. A. Gonis and G.M. Stocks, Plenum (New York, 1992) p. 231.

M.A. Van Hove, "Electron Holography," in Scientific Highlights, Ed. F. Nicodemi, World Scientific (Singapore), p. 137 (1993).

M.A. Van Hove, "LEED, Forward Focusing and Electron Holography," MSA Bulletin 23, 119 (1993).

P.M. Len, S. Thevuthasan, C.S. Fadley, A.P. Kaduwela, and M.A. Van Hove, "Atomic Imaging by $X$-Ray Fluorescence Holography and Electron Emission Holography: A Comparative Theoretical Study," Bull. Am. Phys. Soc. 38, 364 (1993); and submitted to Phys. Rev. Lett.

S. Thevuthasan, S. Ruebush, E.D. Tober, R.X. Ynzunza, F. Zhang, C.S. Fadley, A.P. Kaduwela, Y.J. Kim, and M.A. Van Hove, "Optimization of Data Acquisition and Data Analysis in Photoelectron Holography," Bull. Am. Phys. Soc. 38, 364 (1993). 


\section{Scanning Tunneling Microscopy of Heterostructures and Interfaces in Cross Section}

Principal Investigator: Eicke R. Weber

Funding: $\$ 69,200$ (FY 93)

$\$ 104,300$ (FY 92)

Project No. 92023

\section{Project Description}

High-resolution scanning tunneling microscopy (STM) in an ultra-high vacuum (UHV) chamber allows observation at atomic resolution of the top atomic layer of a sample prepared in situ. Moreover, the possibility of obtaining the I/V characteristics of any atomic location makes the STM a spectroscopic instrument. However, the general application of STMs uses "plan-view" geometry for surface science research, probing only the top few layers, which is not very useful for the study of thin-film heterostructures. For such structures it is of great interest to study cross sections of thin films and interfaces created by in-situ cleavage in UHV. This allows to directly image and analyze with atomic resolution the electronic structure of thin films such as quantum confined structures and interfaces such as metal/semiconductor interfaces or weak links (SIS or SNS type) in superconductors. In the last decade, cross-sectional transmission electron microscopy (TEM) has brought about a breakthrough in the study of the atomic structure of heterostructures; it is expected that the cross-sectional approach in STM and later in the related atomic force microscopy (AFM) will open up new horizons as well.

During the second year of this project, the UHV chamber was completed and the first X-STM images obtained in situ in UHV were obtained in May 1993. A noise test was obtained with a GaAs sample cleaved in situ in UHV. An extraordinary low noise in zdirection (of the order of $0.005 \AA$ ) was evident. This extraordinary performance has to be ascribed to the thermally balanced construction of this setup. In the meanwhile, this type of scanning probe head has been implemented by several other groups in LBL.

The performance of this system exceeded by far our expectations, and several breakthrough-type results were obtained in only a few months. The first result was the first identification of an isolated anion antisite defect in GaAs substrate material. A few weeks earlier (March 1993) a group at IBM had identified this important defect for the first time in As-rich GaAs

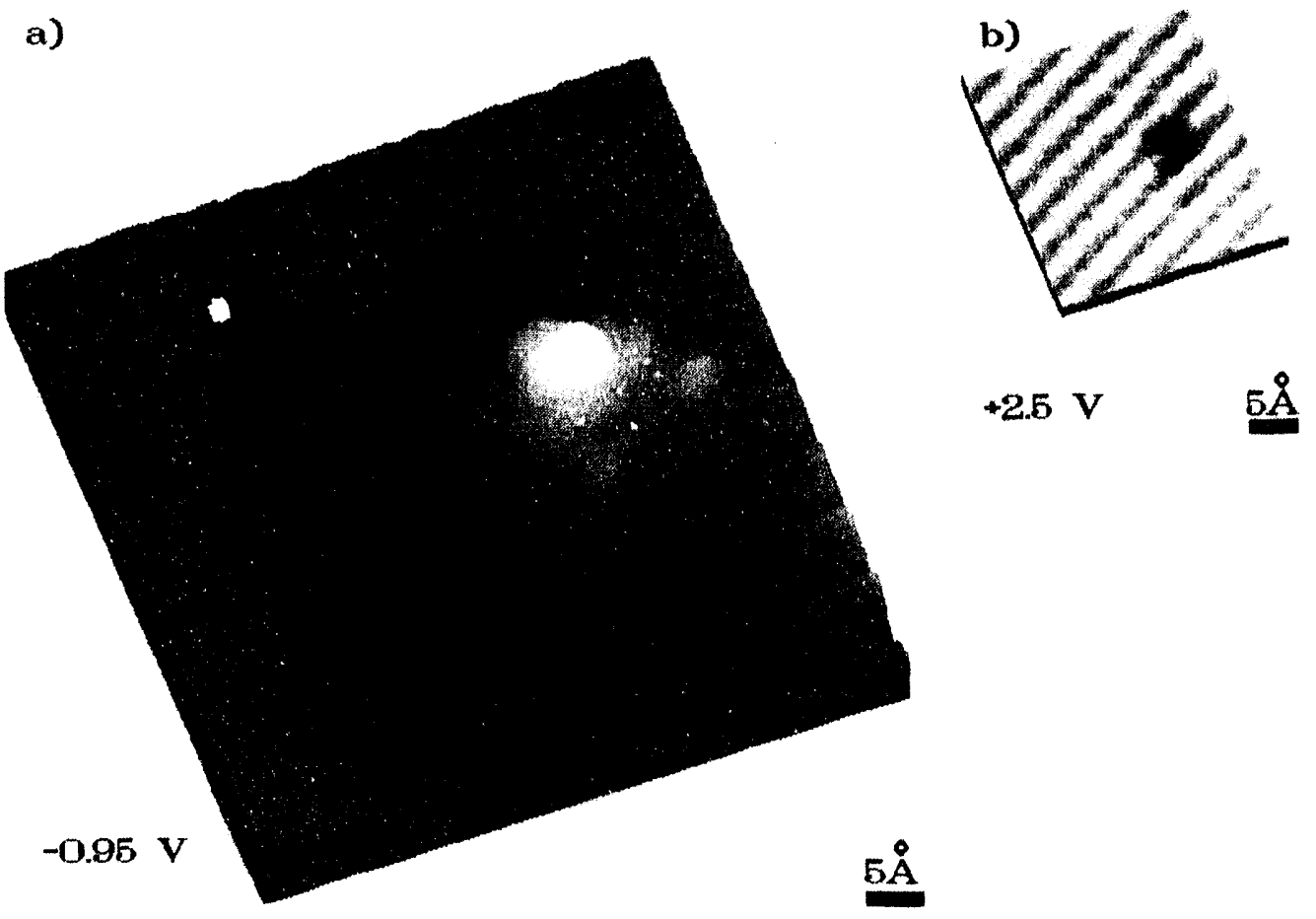

Fig. 11. STM of an isolated As antisite defect in GaAs. Shown is the As sublattice imaged for tunneling out of filled states of the sample, (a) and the Ga sublattice seen in the empty state image (b). Note the Ga vacancy on the site of the extra As atom. 
grown by $\mathrm{MBE}$ at very low temperatures; in such layers, about $10^{20}-\mathrm{cm}^{-3}$ anion antisite defects are formed. Detailed studies were done of one single point defect in the course of an afternoon, revealing, e.g., for the first time the Ga vacancy in which the As atom is located by imaging the $\mathrm{Ga}$ sublattice (Fig. 11). Secondly, the STM image of Si donors in GaAs was identified for the first time. Donor atoms in cubsurface layers can the distinguished from those in the surface layer where they produce localized, dangling-bond type states. Thirdly, the unique, triangle-shaped image of $\mathrm{Zn}$ shallow acceptors in GaAs was identified. Finally, the most exciting achievement is the first observation in atomic resolution of the sublattice atoms of an $\mathrm{InGaAs} / \mathrm{GaAs}$ strained layer quantum well. Figure 12 shows the different tunnel current from In atoms (appearing bright in this constantcurrent image), as compared to the $\mathrm{Ga}$ atoms. The sublattice resolution allows for the first time to study interface roughness on an atomic scale, layer by layer from the surface. Each individual layer is very rcugh, only the averaging of many layers creates an interface. Due to surface segregation, individual In atoms are found high up in the GaAS layer. The In atoms inside the strained layer show a spatial correlation in their appearance, preferentially arranging in pairs and triplets along the growth direction.

These results demonstrate the potential of this new technique and point the direction of further research, which will allow for the first time to study the atomic structure of interfaces atom-by-atom, with the possibility to simultaneously probe the electronic properties of the heterostructure spatially resolved.

Jun-Fei Zheng, the graduate student involved in this project, received a Materials Research Society outstanding graduate student award for his paper "Atomic Scale Interface Structure of InGaAs/GaAs Strained Layers Studied by Cross-Sectional Scanning Tunneling Microscopy."

\section{Publications}

J.F. Zheng, X. Liu, E.R. Weber, D.F. Ogletree, and M. B. Salmeron, " $\mathrm{Si}_{\mathrm{Ga}}$ Donors Observed by Scanning Tunneling Microscopy," to be published in J. Vac. Sci. Technol.

J.F. Zheng, D.F. Ogletree, J.D. Walker, M.B. Salmeron, and E.R. Weber, "Cross-Sectional Scanning Tunneling Microscopy of Semiconductor Surface Emitting Laser Structures," to be published in J. Vac. Sci. Technol.

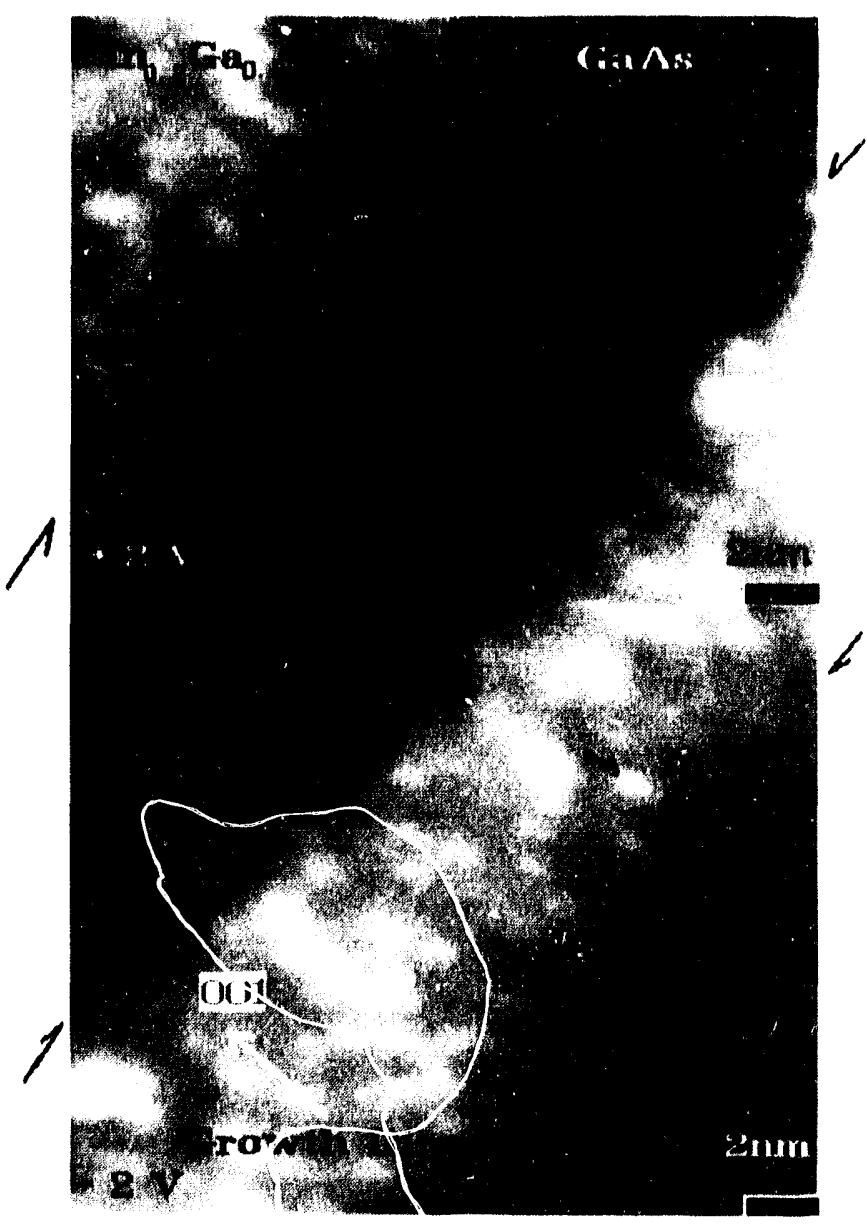

Fig. 12. Cross-sectional tunneling microscopy of a strained InGaAS/GaAS layer; In atcms appear bright due to enhanced tunreling from their sites. Note the roughness of the interfaces in layer-by-liyer resolution, the largest roughness of the upper interface when individual In atoms are carried up far into the $\mathrm{GaAs}$, and the correlation of the arrangemert of In atoms inside the InGaAS layer.

J.F. Theng, X. Liu, N. Newman, E.R. Weber, D.F. Ogletree, and M. B. Salmeron, "Scanning Tunneling Microscopy Studies of Shallow Donor $\left(\mathrm{Si}_{\mathrm{Ga}}\right)$ in $\mathrm{GaAs}$," submitted to Phys. Rev. Lett.

J.F. Zheng, J.D. Walker, M.B. Salmeron, and E.R. Weber, "Clustering and Segregation in Strained-Layer InGaAs/GaAs Heterostructure Studied by CrossSectional Scanning Tunneling Microscopy," submitted to Phys. Rev. Lett.

J.F. Zheng, M.B. Salmeron, and E.R. Weber, "Empty and Filled State Images of Shallow Acceptor $\left(Z_{n_{\mathrm{Ga}}}\right)$ in GaAs Studied by Scanning Tunneling Microscopy," submitted to Appl. Phys. Lett. 
J.F. Zheng, M.B. Salmeron, and E.R. Weber, "Atomic Scale Interface Structure of InGaAs/GaAs Strained Layers Studied by Cross-Sectional Scanning Tunneling Microscopy," Materials Research Society Fall Meeting, Boston, MA, November 1993, in press.

J.F. Zheng, M.B. Salmeron, and E.R. Weber, "STM Studies of Isolated Antisite Defects ( $\mathrm{As}_{\mathrm{Ga}}$ ) in Bulk $\mathrm{n}^{+}$ GaAs," in preparation.

J.F. Zheng, M.B. Salmeron and E.R. Weber, "Dopant Induced Layer Disordering of Molecular Beam Epitaxially Grown AlAs/GaAs Short Period Superlattice in Surface Emitting Laser Structure," in preparation.

J.F. Zheng, M.B. Salmeron, and E.R. Weber, "Direct Evidence of Metal-Induced-Gap-States at Metal/GaAs (110) interface," in preparation.

\section{New Fullerene-Based Materials}

Principal Investigators: Marvin L. Cohen, Joel M. Hawkins, Yuen-Ron Shen, and Alex Zettl

Funding: \$142,900 (FY 93) Project No. 92034 $\$ 198,400$ (FY 92)

\section{Project Description}

The goal of this project has been to experimentally and theoretically explore the properties of novel fullerene-based materials. Single crystals of $\mathrm{C}_{60}$ doped with alkali metal form new conductors and superconductors. Functionalized molecules are synthesized and their properties in thin film, powder, and single crystal form explored. Tubes based in carbon and other materials are synthesized and characterized via transmission electron microscopy (TEM) measurements. Electronic band structure calculations are used to predict electronic properties.

\section{Accomplishments}

Thin films, powders, and single crystals of various fullerenes (including $\mathrm{C}_{60^{-}}$and $\mathrm{C}_{70}$-based structures and fullerene-based tubes) have been prepared. The materials have been characterized by structural ( $x$-ray diffraction), conductivity, tunneling, elasticity, TEM, and mechanical (Young's modulus) measurements.
Rubidium, potassium, cesium, lithium, and calcium doping of the single crystals has been carried out, and these samples have formed the basis of several transport studies.

Hall effect studies show an unusual relationship between Hall coefficient and lattice constant. The electrical resistivity of $\mathrm{K}_{3} \mathrm{C}_{60}$ and $\mathrm{Rb}_{3} \mathrm{C}_{60}$ has been measured up to $900 \mathrm{~K}$, and resistivity saturation has been observed in $\mathrm{Rb}_{3} \mathrm{C}_{60}$. This establishes the intrinsic scattering length ( $\mathrm{C}-\mathrm{C}$ bond length). The constantvolume resistivity has been determined by pressure measurements; a linear temperature dependence is observed that puts limits on the electron-phonon coupling constant.

The rubidium isotope effect has been determined to high precision in $\mathrm{Rb}_{3} \mathrm{C}_{60}$. No isotopic dependence to $T_{c}$ is found, which puts constraints on the role played by the alkali optic modes in the superconductivity mechanism.

A novel mechanical resonator has been developed, and the elasticity of pure $\mathrm{C}_{60}$ films measured over a wide temperature range. Structural phase transitions has been studied at $160 \mathrm{~K}$ and below $100 \mathrm{~K}$.

Fullerene-like tubes have been synthesized from pure carbon and from mixtures of carion, boron, and nitrogen. These tubes have been identified via highresolution TEM studies.

\section{Publications}

J.G. Hou, X.-D. Xiang, M.L. Cohen, and A. Zettl, "Granularity and Upper Critical Fields in $\mathrm{K}_{3} \mathrm{C}_{60}$," submitted to Physica C.

A. Zettl, L. Lu, X.-D. Xiang, J.G. Hou, W.A. Vareka, and M.S. Fuhrer, "Normal State Transport Properties of Fullerene Superconductors," Physica C, in press.

J.G. Hou, X.-D. Xiang, A. Zettl, and M.L. Cohen, "High Temperature Resistivity Saturation in Fullerene-Based Superconductors," submitted to Phys. Rev. Lett.

J.G. Hou, X.-D. Xiang, V.H. Crespi, M.L. Cohen, and A. Zettl, "Magnetotransport in Single Crystal $\mathrm{Rb}_{3} \mathrm{C}_{60}$," submitted to Phys. Rev. B.

W.A. Vareka and A. Zettl, "Linear Temperature Dependent Resistivity at Constant Volume in $\mathrm{Rb}_{3} \mathrm{C}_{60}$," submitted to Phys. Rev. Lett. 



\section{Nuclear Sciences Division}

\section{Laser Trapping of Neutral Radioactive Atoms}

Principal Investigator: Stuart Freedman

Funding: \$185,100 (FY 93)

Project No. 92035

$\$ 99,600$ (FY 92)

\section{Project Description}

The discovery that neutral atoms can be effectively manipulated with laser light signaled a revolution in low-energy experimental physics. Now neutral atoms are routinely cooled and trapped with lasers, but the revolution has only begun. The observation that the ultimate effective temperature of the cold trapped atoms is orders of magnitude below original expectations is leading to a new picture on the behavior of atoms in intense electromagnetic fields. For high densities the motion of the atoms themselves is effected by the force from re-radiated photons, and there is evidence of interesting collective phenomena. The ability to study the interactions among atoms at a few micro-Kelvin is providing probes of the long-range components of the interatomic force. It may be possible to form a condensate of bosonic atoms. Most exciting for some, however, are the new and unforeseen opportunities for applying the new technique to a variety of high-precision measurements.

In particular the new techniques for manipulating cold neutral atoms have promising applications in fundamental nuclear and particle physics research. Two years ago, we began a program of experiments with this goal in mind. The first science goal is a precise test of the vector-axial-vector form of the weak interaction from a study of the beta decay of ${ }^{21} \mathrm{Na}$. The test is made by comparing the lifetime of ${ }^{21} \mathrm{Na}$ with a precise measurement of the beta-decay asymmetry. The strategy is to employ a laser-trapped source of optically pumped ${ }^{21} \mathrm{Na}$ atoms to expedite the most precise asymmetry measurement ever attempted. This method allows us to eliminate several systematic problems. The physics issue is timely: there is tantalizing evidence from neutron decay experiments that the weak charged current should be purely $\mathrm{V}-\mathrm{A}$, but a $\mathrm{V}+\mathrm{A}$ current is already being claimed from measurements of neutron beta decay. A decisive experiment could point to the existence of a new multiplet of heavy intermediate vector bosons, and a revolutionary discovery. While this is an exciting possibility, the techniques that are being developed will have many other applications even if the right-handed current from existing experiments is only an illusion.

\section{Accomplishments}

During the current year significant progress was made developing new techniques of atom manipulation and understanding the physics of atom trapping in general. Most of this work was done in experiments with stab' $c$, rather than radioactive, sodium. A new method was discovered for trapping sodium in the lowest $\mathrm{F}=1$ component of the ground state, and a new $F=1$ to $F^{\prime}=1$ trapping transition was demonstrated. In this trap hyperfine changing collision losses are eliminated. The trap is a surprise because it involves a noncycling transition, but the experiments conclusively demonstrate the nature of the new trap. A general study was also carried out of collision losses in sodium and hyperfine changing collisions of sodium was observed in a $\mathrm{F}=2$ to $\mathrm{F}^{\prime}=3$ trap for the first time. A report of this work has been submitted to Physical Review Letters.

We now hold the world's efficiency record for trapping atoms from a beam. This feat is an essential milestone toward radioactive atom trapping. Using a new strategy for magnetic slowing, we were able to trap $20 \%$ of the beam that passes through the trap. The previous record, held by an MIT group, was less than $2 \%$. The highly efficient trapping method has already expedited a series of sensitive studies of trapping and cooling. One example is the discovery of traps involving noncycling transitions.

Achieving 20\% trapping efficiency is only one of the tools required for radioactive atom trapping. This year we demonstrated that by transversely cooling the atomic beam we can increase the axial flux by a factor of 20. The techniques for producing intense ${ }^{21} \mathrm{Na}$ beams were refined early in the year at the 88Inch Cyclotron. The radioactive isotope is produced with the ${ }^{24} \mathrm{Mg}(\mathrm{p}, \alpha)^{21} \mathrm{Na}$ reaction using magnesium contained in a heated oven being bombarded by 
$30 \mathrm{MeV}$ protons. The radioactive beam was transversely cooled but not with the new sophisticated method recently developed.

In our most recent cyclotron run we accomplished the most important milestone in this program becoming the first group to collect radioactive atoms in a laser trap. The feasibility of our approach has been demonstrated. We are now exploring some immediate applications and proceeding with the ${ }^{21} \mathrm{Na}$ asymmetry experiment.

\section{Publications}

S.-Q. Shang, Z.-T. Lu, and S.J. Freedman, "Comparison of the Cold-Collision Losses for Laser Trapped Sodium Atoms in Different Ground Hyperfine Sub-Levels," submitted to Phys. Rev. Lett.

Z.-T. Lu, S.-Q. Shang, and S.J. Freedman, "An Efficient Method for Loading A Magneto-Optical Trap with a Slowed Atomic Beam," submitted to Phys. Rev. Lett.

Z.-T. Lu, C. Bowers, S.J. Freedman, B.K. Fujikawa, J.L. Mortara, S.-Q. Shang, K.P. Coulter, and L. Young, "Laser Trapping at Short-Lived Radioisotopes," submitted to Phys. Rev. Lett.

\section{New Concepts in IsoSpin Studies}

\author{
Principal Investigator: J. Michael Nitschke \\ Funding: $\$ 130,600$ (FY 93) Project No. 93037 \\ $\$ 63,400$ (FY 92)
}

\section{Project Description}

The purpose of this study is to evaluate the feasibility of establishing a new, unique tool for nuclear physics research-the IsoSpin Laboratory (ISL). The ISL will open up a new era in nuclear physics with unique and exciting scientific opportunities for the nuclear physics community as a whole and the Laboratory in particular. The ISL will facilitate nuclear reactions with beams of radioactive ions (in addition to stable ions) and thus expand nuclear physics research in the new dimension of isospin. There is a chance that the ISL could be built at this Laboratory because of its outstanding record in accelerator-based nuclear science and the availability of the Bevalac complex.
However, several site-specific questions related to the unique location and infrastructure of LBL have to be addressed.

1. What is the optimum design for a high-intensity, primary beam accelerator?

2. What is the best method for accelerating lowvelocity, low charge-to-mass-ratio ions?

3. What are the environmental and radiological concerns of siting the ISL at LBL?

\section{Accomplishments}

An important issue is to find a cost-effective way to produce the primary light-ion beams that are needed for the first stage of the ISL. After consultation with several accelerator experts the present conclusion is that a cyclotron or a Rapid Cycling Synchrotron (RCS) are the most economical ways to produce these ions. The cost for either one of these options is estimated to be in the range of $30-40 \mathrm{M} \$$. A conceptual design study for both machines has been carried out. The first option, an isochronous ring cyclotron, is capable of accelerating protons from 70 to $600 \mathrm{MeV}$ with intensities of $200 \mu \mathrm{A}$. The cyclotron has six sectors, $2.4 \mathrm{MeV}$ energy gain per turn, and excellent extraction efficiency. The initial $70 \mathrm{MeV}$ beam is produced by a small commercial injector cyclotron. The second option is a $30-\mathrm{Hz}$-cycling synchrotron capable of accelerating $2.1 \times 10^{13}$ protons per pulse to an energy of $600 \mathrm{MeV}$ resulting in an average current of $100 \mu \mathrm{A}$. The RCS is injected by a $70 \mathrm{MeV} \mathrm{H}^{-}$LINAC. The RCS would fit inside the present Bevatron enclosure thus saving considerable funds for shielding and other infrastructure. An important benefit of a dedicated primary accelerator would be that the beam is available $100 \%$ of the time for radioactive beam-or isotope production.

The second task was to find the best method for accelerating the low-velocity, low-charge-to-mass ratio radioactive ions to energies of several $\mathrm{MeV} / \mathrm{u}$. A workshop was convened at LBL to discuss postaccelerator issues at the ISL such as ion sources, electromagnetic separators, RFQs, and low- $\beta$ structures. The new post-accelerator concept that resulted from this workshop is shown in Fig. 1, where the key improvement over earlier versions is that the first RFQ no longer has to operate at high-voltage potential. The matching between the constant energy beam from the ion source/separator and the required constant velocity at the input of the RFQ is achieved with a matching $\mathrm{rf}$ cavity with variable energy gain. Another new development is the Broad Range Atomic 


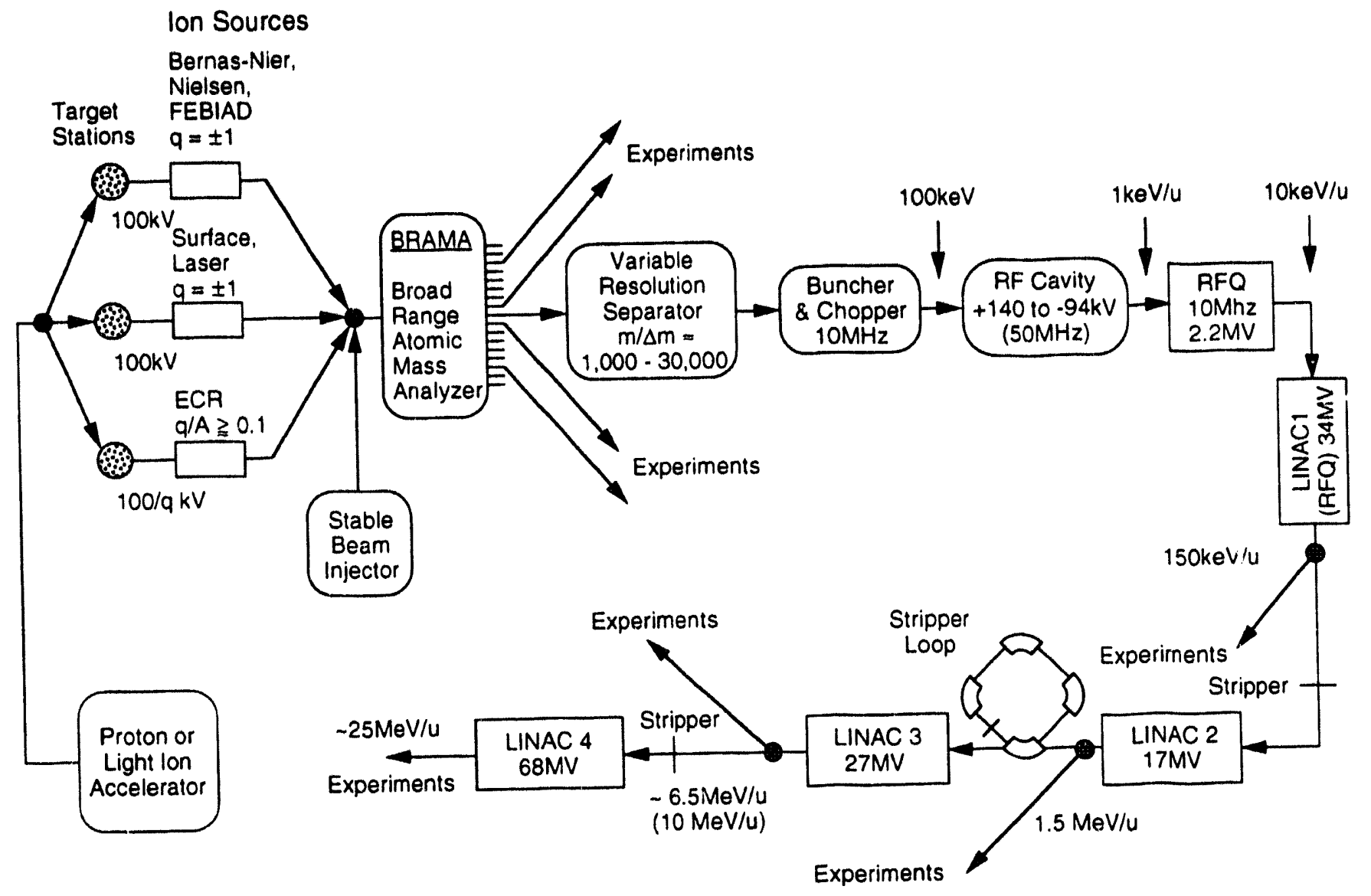

Fig. 1. Current version of the IsoSpin Laboratory concept. New ideas incorporated during the present report period are the Broad Range Atomic Mass Analyzer (BRAMA), the Buncher/Chopper, the If matching cavity, and the Stripper Loop.

Mass Analyzer (BRAMA). It consists of a large magnetic spectrometer that analyses the entire mass spectrum of radioactive ions simultaneously. BRAMA will allow many experiments to be carried out concurrently, thereby improving the user multiplicity of the ISL several fold.

Environmental and radiological concerns are of paramount importance in considering the siting of the ISL at LBL. Studies by a consultant and calculations at LBL have been carried out. These calculations show that $\sim 5 \mathrm{~m}$ of steel or $\sim 10 \mathrm{~m}$ of concrete are required to shield the primary target area. Much of the shielding material for the accelerators and beam lines is avai'able from the Bevatron $\left(\sim 10^{4}\right.$ tons of steel and $\sim 10^{5}$ tons of concrete). Calculations of radioactive inventories show that DOE Category 3 limits will be exceeded for high- $\mathrm{Z}$ targets mainly due to the production of ${ }^{125} \mathrm{I}$ and ${ }^{131} \mathrm{I}$. This would make the ISL a Category 3 nuclear facility. Radiation fields near the target will exceed $10^{4} \mathrm{rads} / \mathrm{h}$ at $1 \mathrm{~m}$ and will require remote handling. To avoid air activation, the target area will be evacuated. Additional topics addressed in the radiological studies are waste disposal and storage, seismic safety, and operational procedures.

Several other results were accomplished during this report period:

- A target test stand has been completed and exceeded, with $50 \mathrm{~kW}$, its design-power goal of $40 \mathrm{~kW}$.

- A new gas-cooled target concept has been tested with a stainless steel target that was operated at $10 \mathrm{~kW}$. An international collaboration will further exploit this concept during target tests at Rutherford-Appleton Laboratory in England.

- A computer model for an enhanced fission target is under development. This ${ }^{235} \mathrm{U}$ target would increase the intensities of fission-fragment beams by several orders of magnitude. 
To avoid beam losses in the ISL, lattice calculations for a "charge state enforcer" ring have been carried out. The device could be inserted between one or two stages of the post-accelerator (c.f. Stripper Loop in the Fig. 1) and could increase radioactive beam intensities by as much as a factor of 20 .

\section{Publications}

Y. Jongen, D. Vanderplassche, and G. Lannoye, Internal Report, Ion Beam Applications Publishers, Belgium (1993).

Proceedings of the Workshop on Post-Accelerator Issues at the IsoSpin Laboratory, edited by S. Chattopadhyay and J.M. Nitschke, to be published.

J.M. Nitschke, "Technical Issues at the IsoSpin Laboratory," presented at the 206th American Chemical Society National Meeting, Division of Nuclear Chernistry and Technology, Symposium on Technical and Scientific Issues of Radioactive Beams, Chicago, IL, August 22-27, 1993.

J.M. Nitschke, "BRAMA, a Broad Range Atomic Mass Analyzer for the ISL," Workshop on Post-Accelerator Issues at the IsoSpin Laboratory, Berkeley, 1993, edited by S. Chattopadhyay and J.M. Nitschke, to be published.

"IsoSpin Laboratory Radiation Assessment," M.M. Barbier Inc., Herndon, VT, Report No. MMB- 53 (1992).

R.J. Donahue, Workshop on the Production and Use of Intense Radioactive Beams at the IsoSpin Laboratory, Oak Ridge TN, 1992, edited by J. D. Garrett, CONF9210121.

\section{Microstrip Gas Chambers for TPC Readout}

Principal Investigators: Howard H. Wieman, Wen G. Gong, John W. Harris, and Jeffery T. Mitchell

Funding: $\$ 103,700$

Project No. 93021

\section{Project Description}

Traditionally, Time Projection Chambers (TPCs) have been read out with multiwire proportional chambers
(MWPCs) located over a surface of pads that pick up the induced signal from avalanches on the wires. This MWPC technology sets a practical limit on the two track resolution and the position resolution that can be obtained with a TPC. The new Microstrip Gas Chamber (MSGC) devices can overcome this limit and allow TPCs to be operated in much higher track density environments with improved position resolution. These devices are not of much benefit in large TPCs where transverse diffusion is large. In small TPCs with cool gases and short drift distances, however, the high-density spatial resolution of the MSGC is well matched to the small diffusion widths. The purpose of this project is to develop MSGCs suitable for a practical TPC readout. In the course of this work we have expanded the scope of our effort to include:

- Investigation of MSGCs constructed on a thin substrate for use as tracking detectors

- Investigation of MSGCs on a transparent substrate backed with a photocathode surface as a possible economic alternative to microchannel plates

- Building and testing Bellazzini's Micro Gap Chamber.

The scope of the project was expanded when it was discovered that through the use of the Berkeley Microfabrication Laboratory we could investigate many more chamber designs than originally thought possible.

\section{Accomplishments}

MSGCs were constructed on a variety of substrates. The geometry is shown in Fig. 2. Three types of glass were used, Corning C7740, Schott S8900, and fused silica. The $589 \mathrm{CO}$ glass is a special low-resistance glass developed for Pestov Spark Chambers. The low resistivity is desirable for high-counting rate detectors because it reduces surface charge buildup that can affect the gain of the detector. The fused silica backing was chosen for tests of the alternative microchannel plate idea since it is transparent to UV, thereby permitting transmission of a broader spectrum of photons from the avalanche to the photocathode surface. Two alumina ceramic substrates, Kyocera Ceramics A445 and A201, were also investigated. Ceramics are of interest because ceramic packaging is widely used in the electronics industry, and construction of MSGCs on ceramics is a potential solution to the challenging problem of connecting high-density chambers to electronics. MSGCs were also fabricated on a thin silicon substrate. 


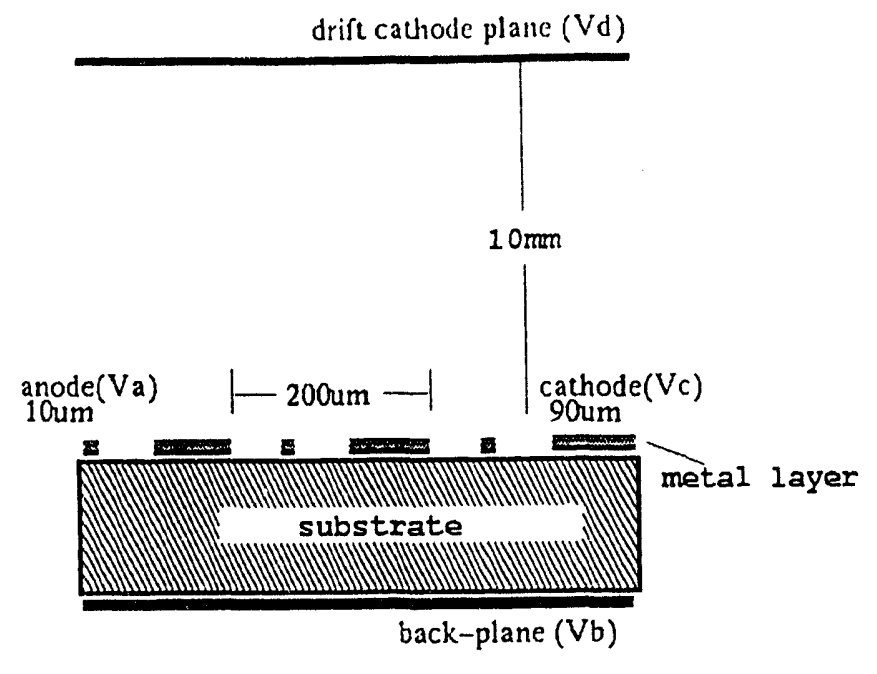

Fig. 2. The structure of a MSGC.

Chambers were constructed with a variety of electrode metals including gold and aluminum. A plasma etch was used to define the electrodes since it provides the best line uniformity. Ion implantation was used to reduce the surface resistivity of the alumina and fused silica substrates.

A test facility was built for operating the MSGCs in $\mathrm{Ar}$ $90 \%+\mathrm{CH}_{4} 10 \%$ with an ${ }^{55} \mathrm{Fe} x$-ray source. Gas gains were measured as a function of bias conditions, and gain stability was studied at high count rates. Detector resolution and maximum gain values were determined. MSGCs on C7740, 88900 glasses, and the A201 alumina substrate have been successfully tested. A good, pulse height histogram taken with a ${ }^{55} \mathrm{Fe}$ source is shown in Fig. 3 for a MSGC constructed on

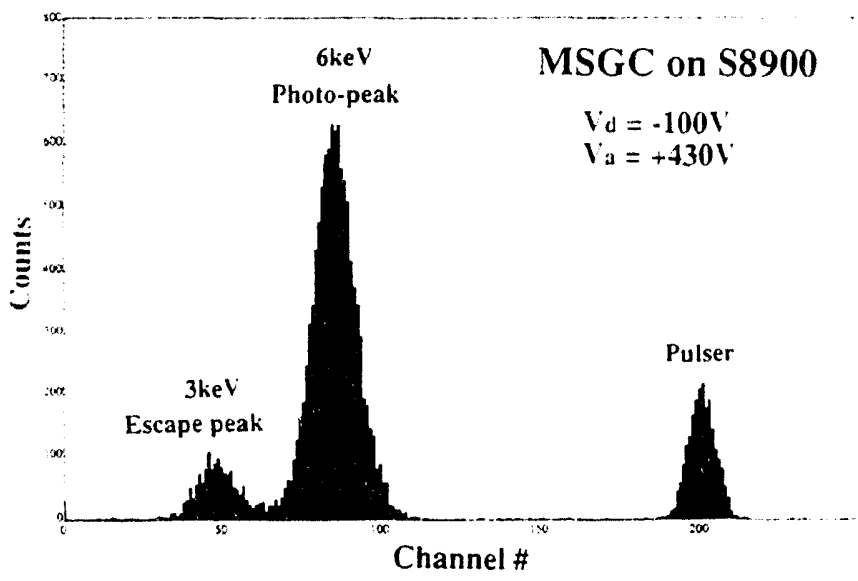

7.. 3. Pulse height spectrum for ${ }^{55} \mathrm{Fe} x$-rays measured with a MSGC constructed on a 58900 glass substrate.
S8900 glass. A microgap chamber has been constructed but testing awaits the completion of a test facility that is safe for use with flammable gas since these chambers must be operated with highly quenched gases. The UV transparent chamber with a photocathode back plane is still under construction.

Tests of the MSGCs constructed with glass substrates, S8900 and C7740, show that these devices are suitable for use in a TPC. The gains were stable at the highest count rates tried, $50000 \times$-ray photons $/\left(\mathrm{mm}^{2} \mathrm{sec}\right)$. This ionization loading is considerably higher than what will be encountered in a TPC application, since space-charge distortions will limit TPC performance long before the readout chambers are subjected to this load. The maximum gas gain achieved before sparking in the tests of these MSGCs was 1000. This is low, but sufficient for the TPC readout application. There are several known improvements in the design that are expected will lead to an increased gas gain. However, merely using a gas with more quencher should boost the gains well beyond what is required.

Attempts to build MSGCs on ceramic have not produced devices that work well as the MSGCs on glass. The low-resistivity alumina substrate, A445 from Kyocera, is too porous to produce the polished surface required for photolithography, so no MSGCs were tested with this material. The high-resistivity alumina, A201 from Kyocera Ceramics, can be polished to a good surface that is suitable for this work, but our devices had unstable gain due to surface charging. Ion implantation was tried, but the dosage apparently was not sufficient to achieve the desired results. Further work, however, could turn ceramic MSGCs into practical TPC readouts. There are recent reports of inexpensive surface coatings on glass MSGCs that can reduce the surface charging effect significantly.

\section{Publications}

W.G. Gong, H. Wieman, J.T. Mitchell, J.W. Harris, W.S. Hong, V. Perez-Mendez, "Microstrip Gas Chambers on Glass and Ceramic Substrates," to be published in IEEE Trans. on Nucl. Sci., June 1994.

W.G. Gong, H. Wieman, J.W. Harris, J.T. Mitchell, "Microstrip Gas Counters on a Membrane Substrate," Bull. Am. Phys. Soc. 38, 1837 (1993).

j. T. Mitchell, W.G. Gong, H. Wieman, J.W. Harris, "Development of Microstrip Gas Chambers as a Time Projection Chamber Readout," Bull. Am. Phys. Soc. 38, 1837 (1993). 



\section{Physics Division}

\section{The Portable DAQ: A Data Acquisition System for Particle Physics Based on a Standardized Software Bus}

Principal Investigators: Gerald S. Abrams, Alessandra Ciocio, and Patrick LeDu

Funding: $\$ 149,600$

Project No. 93022

\section{Project Description}

The software for future large-scale particle physics data-acquisition systems will require extensive developments due to the complexity and diversity of the demands on such a system. There are several competing standards for the hardware data crates, and none to speak of for the software. Compounding these difficulties is the requirement that the bulk of the coding for these experiments be contributed by physicists and students, rather than professional programmers.

It is necessary to produce a standard environment in which physicists can develop software for large-scale data acquisition. The methodology to allow the integration of modular, transportable computer code is in its infancy, and must be developed. This "firmware" will be the basis for the integration of existing software. We propose to specify and deliver, together with our collaborators, a full-scale skeletal implementation of an operational system. While the present scope of work is intentionally limited, the system to be developed here may well have wideranging applicability.

We proposed to implement a software bus, which will provide the necessary standardization for the modular program development essential for projects international in scope. A framework will be constructed to define the data structure, the interface to move and manipulate the data, and the control system for both real-time acquisition and processing/monitoring. The standardization, along with the implementation of development interfaces, will allow the collaborators to proceed in parallel in both the adaptation of working code and the development of new software.

\section{Accomplishments}

An extensive investigation was pursued into existing or contemplated data acquisition and control systems, with the goal of identifying reusable ideas, code, or even entire subsystems. The basic conclusion from this study was that only one of the candidate systems was designed to be sufficiently general and flexible for our needs. The main reasons for this failure seem to reside in the self-imposed constraints or boundary conditions of the developers. In particular, architecture bound to specific hardware technology, and/or adaptation to specific software packages restricts the overall adaptability of these systems.

The Experimental Physics and Industrial Control System (EPICS), in contrast, abundantly fulfills the requirements. Developed by DOE laboratories (ANL and LANL), EPICS provides the flexible and modular framework that allowed us to design and implement a skeletal data-acquisition system based on its slow control and monitoring. This skeletal system incorporates the software bus of EPICS, and extends it into the real-time domain by appending $D A Q$ processes for front end readout, an event builder to merge and order the fragments of each event, and a configuration database to allow flexibility/ modularity in the hardware readout. 


\section{New Si Microstrip Disk Detector for Vertex Reconstruction of B Meson Decays}

Principal Investigators: Alan R. Clark, Frederick

R. Goozen, Natalie Roe, Bruce A. Schumm, and Mark W. Strovink

Funding: $\$ 135,000$ (FY 93)

$\$ 178,000$ (FY 92)

Project No. 92039

\section{Project Description}

To meet new demands for precise 3-dimensional reconstruction of vertices from decays of $B$ mesons produced at forward angles, it was proposed to develop a new type of vertex detector, the Silicon Microstrip Disk (SMD). It consists of wedge-shaped double-sided Si microstrip detectors that are joined at the edges to form an open polygon, supported and read out at the outer radius. New techniques to be developed include reading out a Si detector with variable strip length, reading out both sides of a 2sided detector from the same edge, mounting of the readout electronics directly on the $\mathrm{Si}$ detector, and cooling and support of the disk from the outer radius. Geometries suitable for both hadron colliders and asymmetric $\mathrm{e}^{+} e^{-} \mathrm{B}$ factories will be studied, and a readout architecture appropriate to the given bunch crossing interval for each type of accelerator will be developed.

\section{Accomplishments}

Substantial progress has been made in FY 1993 towards meeting the goals outlined above for the SMD detector development project. The most significant achievements are:

\section{Electronic Readout Circuit}

A flexible kapton circuit, the high-density interconnect '(HDI), has been built and tested, and will be mounted directly on the silicon detector. The HDI provides power and control signals for the readout chips, which are mounted on it and then wire-bonded to the detector. The first prototype was delivered in FY 1992 and has been tested in FY 1993. It had acceptable performance when loaded with the full complement of eight SVX " $D$ " chips. A small oscillation on the analog-out signal was observed and observed to worsen as the total current draw increased. The oscillation was fully damped before the sampling region of the analog-out and hence did not affect chip performance; however, it led to redesigning of the power and grounding of the circuit. The new layout employed blind vias for the signal interconnects, thus avoiding the interruption of the power and ground planes present in the original design. The new layout has been fabricated and tested, and shows improved performance on the analog-out signal.

A third version of the HDI was then fabricated using a new type of flexible kapton material featuring a sputtered-on conductor. This avoids the use of an adhesive layer between the copper conductor and the polyimide, reducing thickness, mass, and improving the thermal conductivity of the circuit. This version has also been tested and shown tc have satisfactory performance.

An HDI is presently being mounted directly on top of an active silicon detector. A first effort led to a technique for positioning and gluing the circuit on the detector without damaging either the circuit or the detector surface. However, the circuit was not positioned with sufficient accuracy, and there was difficulty in wirebonding it to the detector. A more accurate technique is being developed employing the Smart-scope that will allow us to position the HDI a precise distance from the detector wire-bond pads. The detector will then be stimulated by a laser, already set up in the lab and tested with detectors that are mounted on test boards. This will provide a proof-of-principle that readout electronics can be successfully mounted on low-mass circuitry directly on the active silicon detector.

\section{Electronic Readout Cable}

The cables that connect the HDI to the control board have also been designed and prototyped. They are flexible kapton microstrip cables consisting of two layers of aluminum conductor to provide power and ground and a third layer of fine copper traces on a 0.10 in. pitch to bring in control lines and bring out detector signals. Special connectors using low-mass materials have also been designed and tested. Cables up to 10 feet long, including the connector, have been tested. Risetime of the cables is less than $3 \mathrm{~ns}$. This is more than adequate for the anticipated readout at $53 \mathrm{MHz}$. A mockup of a complete disk has been built showing how all wedges are connected, using one cable to service two double-sided detectors.

\section{Mechanical Support}

A spaceframe consisting of small diameter, thinwalled aluminum tubing was designed in FY 1992. This spaceframe provides support for the silicon 
barrel and disk detectors as well as precise alignment of the detectors. Model sections of the frame were produced by brazing. These failed to meet required dimensional tolerances due to the large differential thermal expansion of aluminum. A new model was constructed using fiberglass reinforced epoxy joints. This sample meets the requirement of being within 0.0003 in. rms of the design dimensions. The maximum deviation is 0.0009 in. It has been tested under load and shows elastic behavior (no creep or yield) which is more than adequate for the detector support.

\section{Factory Conceptual Design}

A conceptual design for a silicon strip detector has been completed, which is optimized for use at an asymmetric $B$ factory. The design employs both barrel and disk style detectors and provides coverage to within 300 mrad of the beamline in the forward direction. Simulations have been carried out of detector resolution that demonstrate silicon microstrip disks are well-suited for forward particle detection in this environment. More recently, we have begun to consider the electronic requirements including the choice of analog vs digital readout, tolerable electronic noise levels, and desirable features such as time-stamping and sparse readout.

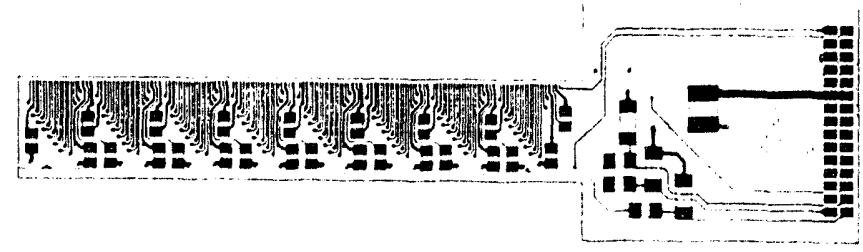

Fig. 1. Revised layout of the high-density interconnect circuit. The actual size is $8 \mathrm{~cm} \times 1.5 \mathrm{~cm}$. The readout chips are located on the eight pads along one side.
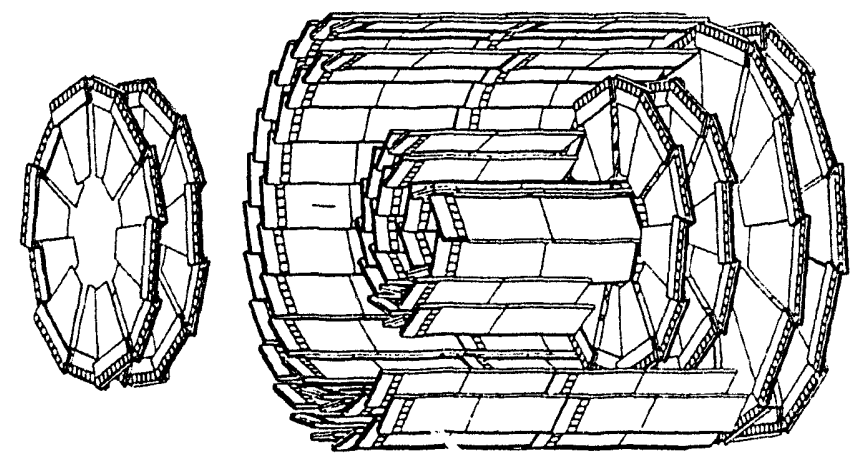

Fig. 2. 3-D view of the silicon detector conceptual design for the asymmetric $B$ factory.

\section{Directions for a Particle Astrophysics Initiative: Additional Observations of the Cosmic Microwave Background}

\author{
Principal Investigator: George F. Smoot \\ Funding: $\$ 103,400$ \\ Project No. 93023
}

\section{Project Description}

Particle physics and cosmology/astrophysics have evolved towards each other; the cross-fertilization has reached the point that experiments in one field can be justified, based upon their impact on the other. In particular, the results obtained from the COBE satellite have energized the study of early-Universe physics. This project is to go beyond these recent achievements to study the early Universe in greater detail. The purpose of this exploratory study was to determine what is necessary to make such measurements, what are appropriate measurements, what one could learn from them, and how well the measurements need to be made.

Inflationary models of cosmology are the only existing models that can explain the COBE and large-scale structure observations. Inflation not only makes the local universe flat, isotropic, and with a near-scaleinvariant spectrum of density fluctuations, but also simultaneously produces gravity waves. The gravity waves have nearly the saine spectrum as the density fluctuations but have an amplitude set by the energy scale of inflation (potential of the inflation field at the time the gravity wave was generated). However, the density perturbations amplitude is set by the ratio of the energy scale of inflation to the derivative of the energy scale (cube of the potential divided by the derivative of the potential). Thus, if one were able to measure the gravity wave spectrum amplitude or its ratio to the density-spectrum amplitude, then one would determine the energy scale of inflation and its derivative.

Learning about the inflation potential is clearly exceptionally interesting physics. It is likely that the energy scale is on the order of $10^{16}$ to $10^{14} \mathrm{GeV}$. The low level of fluctuations observed in cosmic background radiation anisotropy by $C O B E$ indicates that the energy scale of inflation is below a few times $10^{16}$ $\mathrm{GeV}$ at the time current horizon-size fluctuations were generated. The need for baryogenesis set the lower bound above $10^{9} \mathrm{GeV}$. Most current models of infla- 
tion require energy scales on the order of $10^{14} \mathrm{GeV}$ or greater. This is thought to be a very interesting energy range in particle physics. $10^{16} \mathrm{GeV}$ is the predicted energy of grand unification in supersymmetic particle physics theories. It is likely that there will turn out to be a deep connection between grand unification, supersymmetry, and inflation.

\section{Accomplishments}

A number of possible approaches have been investigated. A collaboration was formed with two groups in studies of potential space-based missions. One group is primarily composed of Europeans, and a proposal to ESA (The European Space Agency) was accepted and the feasibility study began in

November. In addition, a workshop on the issues and technology was held the third week in September 1993. Reno Mandolesi of Bologna is the European principal investigator for the proposal along with myself as the American principal investigator. The mission is called COBRAS (Cosmic Background Radiation Anisotropy Satellite). COBRAS is on the scale of a medium cost mission for ESA. We are producing a feasibility study that will cover COBRAS in more detail.

The second group is producing a conceptual feasibility study for a medium class American space mission called PSI (Primordial Structure Investigation). The study scientist chair is Micheal A. Janssen of Jet Propulsion Laboratory. Involved are project managers and spacecraft engineers. Reflecting their differences, the COBRAS proposal focuses primarily upon the instrument and its performance while the PSI study is heavily weighted toward mission feasibility. The PSI mission is pulled by considerations toward a smaller and lower cost mission. At this stage PSI falls between small and medium class missions. The PSI study was scheduled to be completed in June and presented to NASA shortly afterwards. Lack of opportunity for medium class missions has slowed the development but a preliminary report was produced. Small mission opportunities are expected to be available every year from NASA while the next medium class is not expected for at least two years and not for launch before the year 2000.

With graduate student Harold Steinacker, the possible use of cc smic background polarization was investigated as a useful cosmological probe and discriminant. It was found that it is not likely to be as useful as the anisotropy. As part of this effort we interacted with Paul Steinhardt and his collaborators and encouraged them to push forward with determining how much we could learn from the cosmic background anisotropies as they talked to us about polarization and experimental limitations. It was clear that the cosmic background anisotropies are still the cleanest and best cosmological probe we have available.

Fabrication of sample HEMT transistor amplifiers has been arranged to study, test, and prototype the most recent technology. A high school student has been helping test and run a cryogenic refrigerator that will be used for studying the technical feasibility. The general work on future cosmic background radiation anisotropies continues at the present time. 


\section{Structural Biology Division}

\section{Novel X-Ray Crystallographic Studies of RNA}

Principal Investigator: Stephen R. Holbrook

Funding: $\$ 59,000$ (FY 93)

$\$ 68,100$ (FY 92)

Project No. 92041

\section{Project Description}

Despite playing a central role in many cellular processes, little is known about the complex and varied structures that ribonucleic acid (RNA) may assume in order to carry out its biological functions. Although considerable ef fort has been made toward determination of RNA structure, to date, only a few RNA structures have been determined. These have provided a glimpse of the complexity that these molecules may assume. However, complex RNA structural elements such as pseudoknots, hairpin loops, parallel and multistranded helices, and unusual tertiary base pairing schemes have yet to be explored by $x$-ray crystallography. Now that methods of RNA synthesis are at hand, whereby large quantities of highly purified and homogeneous material can be obtained, advances in the methods of crystallization and crystal structure determination are needed in order to further this field.

This project focuses on developing crystallization and structure solution techniques for application to RNA structure determination by $x$-ray crystallography. Studies in FY 1993 have concentrated on collecting $x$-ray diffraction data and testing methods of structure solution. Our efforts in the development of crystallization methods were continued in the second year of support. These methods were tested by application to structural studies of: (1) sequences forming mismatched base-pairs, or internal loops; (2) RNA oligomers that can form pseudoknot structures; (3) RNA elements that serve as protein recognition sites, i.e., the TAR RNA and RRE RNA of the HIV-1 virus, Loop E of 5S RNA and the IRE (Iron Regulatory Element) RNA; and (4) a fragment of the hairpin catalytic RNA.

\section{Accomplishments}

We were able to obtain several suitable crystals for structure analysis. Low molecular weight polyethylene glycol was identified as the most useful precipitant in the crystallization of RNA oligomers. $X$-ray diffraction data were collected on several RNA oligonucleotides, and the crystal structure of a iecond RNA oligomer was determined. The progress is detailed below.

\section{Development of Crystallization Techniques}

A set of 48 trial crystallization screening conditions analogous to the protein crystallization screens was developed, but designed to include conditions previously shown to induce RNA or DNA crystallization. Attempts to crystallize over 25 RNA oligomers with this matrix and the screening matrix of Jancarik and Kim (see Table 1) have resulted in crystallization of 7 different oligomers containing internal loop forming sequences that have been shown to diffract $x$-rays to reasonable resolution. Diffraction data have already been collected on six of these and will soon be available for the other one. In addition, we have obtained single crystals of two other RNA oligomers which are near the size necessary for data collection. Examination of the conditions that have been successful in inducing RNA crystallization showed that 7 of the 9 RNAs crystallized were obtained using PEG 400 as the precipitant. This observation led to the proposal that low molecular weight polyethylene glycols, in particular PEG 400, should be tried as the most likely precipitant for successful crystallization of RNA oligomers. A screening matrix, based on this proposal and focusing on several low molecular weight polyethylene glycols and methoxy-polyethylene glycols, has been designed.

\section{Structure Determination of RNA Oligomers}

In addition to development and testing of RNA crystallization methods and collection of diffraction data on several crystals, we have also been able to refine the structure of one RNA dodecamer and to solve and refine the crystal structure of a second RNA dodecamer. These are described below. 
Table 1. Oligonucleotides for which crystallization was attempted.

\begin{tabular}{|c|c|c|c|}
\hline Sequence & Type & Size & Structural Feature \\
\hline GCCGGAG & RNA & 7 & Internal Loop \\
\hline GCGAGCU & RNA & 7 & Internal Loop \\
\hline CGCAAGCG & RNA & 8 & Internal Loop \\
\hline CGCAGGCG & DNA & 8 & Internal Loop \\
\hline CGCAGGCG & RNA & 8 & Internal Loop \\
\hline CGCAIGCG & RNA & 8 & Internal Loop \\
\hline CGCCAGCG & RNA & 8 & Internal Loop \\
\hline CGCCCGCG & RNA & 8 & Internal Loop \\
\hline CGCCUGCG & RNA & 8 & Internal Loop \\
\hline CGCGAGCG & DNA & 8 & Internal Loop \\
\hline CGCUCGCG & RNA & 8 & Internal Loop \\
\hline CGCUUGCG & RNA & 8 & Internal Loop \\
\hline CTAGACGT & DNA & 8 & Protein Recognition Site \\
\hline GGCAGGCC & RNA & 8 & Internal Loop \\
\hline GGCAGGCC-p & RNA & 8 & Internal Loop \\
\hline GGCGAGCC & RNA & 8 & Internal Loop \\
\hline GGCGAGCC & DNA & 8 & Internal Loop \\
\hline GICGAGCC & RNA & 8 & Internal Loop \\
\hline GGCGPGCC & RNA & 8 & Internal Loop \\
\hline CCCGAAAGGG & DNA & 10 & Tetraloop or Internal Loop \\
\hline CGCGGGGGCG & DNA & 10 & "G Quartet" \\
\hline CGCGIGCGCG & DNA & 10 & Internal Loop \\
\hline CGCGIICCCGCG & DNA & 12 & "G (I) Quartet" \\
\hline GGACTTCGGTCC & DNA & 12 & Tetraloop or Internal Loop \\
\hline GGACUUCGGUCC & RNA & 12 & Tetraloop or Internal Loop \\
\hline GGACUUUGGUCC & RNA & 12 & Tetraloop or Internal Loop \\
\hline GGACUUUGGUCC & RNA & 12 & Tetraloop or Internal Loop \\
\hline GGAGCGAGCUCC-p & RNA & 12 & Internal Loop \\
\hline GGCCGAAAGGCC & RNA & 12 & Tetraloop or Internal Loop \\
\hline RRE (REV.REC.EL.) & RNA & 26 & Internal Loop \\
\hline 5S - Loop E & RNA & 27 & Internal Loop \\
\hline RZ2-RS2 (Catalytic) & RNA & 28 & Internal Loop \\
\hline VP2-PSEUDOKNOT & RNA & 34 & Pseudoknot \\
\hline TAR (TAT BINDING) & RNA & 35 & Bulge, Double Stem-I,oop \\
\hline YEAST(PHE)tRNA & RNA & 76 & Cloverleaf \\
\hline
\end{tabular}

p-GGACUUCGGUCC - The crystal structure of this RNA dodecamer was determined prior to the initiation of LDRD support. Under LDRD funding this crystal structure was refined to clarify structural details, allow location of bound water molecules, and quantify the atomic mobility or flexibility in terms of the crystallographic B-factors. Refinement is particularly important for this crystal structure since the resolution of the diffraction data, $2.0 \AA$, is the highest of any RNA structure to date. This structure provides the first example of a U-C mismatch base pair and illustrates the importance of water structure in stabilization of the non-Watson-Crick pairs formed in RNA internal loops.

GGACUUUGGUCC - The crystal and molecular structure of the double stranded form of this dodecanucleotide incorporating an internal loop has now been determined. The structure was solved by molecular replacement using a modified RNA-A duplex as a probe. Refinement has so far consisted of rigid body, segmented rigid body, and positional and several cycles of X-PLOR simulated annealing. Temperature factors have been refined and the process of locating waters has been initiated. The current $R$ factor is approximately $21 \%$ for data between 12 - and $2.6-\AA$ resolution. The oligomer forms a 12-base pair duplex in the asymmetric unit that incorporates two central U-U pairs flanked by U$\mathrm{G}$ pairs on each side. A stereoview is shown in Fig. 1. The duplex is not symmetric with one of the U-U pairs being highly twisted and the other relatively planar. Packing interactions are through minor groove-minor groove contacts in the direction of thm screw axis. The overall helix is not distorted from :form. The helical rotation is $33.6^{\circ}$ and the displacement $2.71 \AA$. A comparison with the previously determined structure of GGACUUCGGUCC will be informative. 

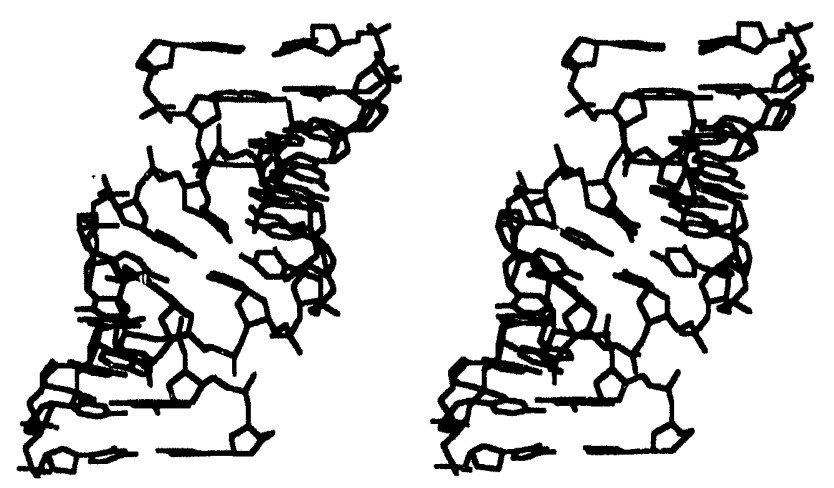

Fig. 1. Stereoview of the structure of the RNA dodecamer (GGACUUUGGUCC) 2 incorporating two $U-G$ and two $U$-U base pairs as an internal loop in the duplex.

\section{Publications}

K.J. Baeyens, J. Jancarik, and S.R. Holbrook, "Use of Low Molecular Weight Polyethylene Glycol in the Crystallization of RNA Oligomers," submitted to Acta Crystallographica, Sect. D.

S.R. Holbrook, J. Yeh, J. Pandit, J. Jancarik, C. Cheong, I. Tinocu Jr., and S.-H. Kim, "Crystal and Molecular Structure of a Non-Complementary RNA Double Helix: [GGACUUCGGUCC] ${ }_{2}$, in preparation.

K.J. Baeyens, H. DeBondt and S.R. Holbrook, "Crystal and Molecular Structure of an RNA Double Helix Including Uracil-Uracil Base-Pairs in an Internal Loop," in preparation. 


\section{Acronyms and Abbreviations}

\begin{tabular}{|c|c|}
\hline AFM & atomic force microscopy \\
\hline $\begin{array}{l}\text { ALS } \\
\text { APM }\end{array}$ & $\begin{array}{l}\text { Advanced Lignt Source } \\
\text { additive pulsed modelocked }\end{array}$ \\
\hline ASSPG & aggregated spin system proximity graph \\
\hline ATM & asynchronous transfer mode \\
\hline B-ISDN & broadband integrated service digital network \\
\hline BRAMA & Broad Range Atomic Mass Analyzer \\
\hline CDRL & Chemical Dymamics Research Laboratory \\
\hline CEBAF & Continuous Electron Beam Accelerator Facility \\
\hline CIS & Constant Initial State \\
\hline COBRAS & Cosmic Background Radiation Anisotropy Satellite \\
\hline CS & cigarette smoke \\
\hline DAS & differential absorption spectroscopy \\
\hline DISC & Direct Injection Stratified Charge \\
\hline DLTS & deep level transient spectroscopy \\
\hline DOE & U.S. Department of Energy \\
\hline DSG & destructive solid geometry \\
\hline DSM & demand-side management \\
\hline EMCS & energy management and control systems \\
\hline EM & electromagnetic \\
\hline EMCS & energy management control systems \\
\hline EPICS & Experimental Physics and Industrial Control System \\
\hline ESA & European Space Agency \\
\hline EXAFS & extended $x$-ray absorption fine structure spectroscopy \\
\hline FEL & free electron laser \\
\hline FMC & fire mode of combustion \\
\hline FTC & Flame Traversing the Charge \\
\hline FWHM & full-width at half-maximum \\
\hline HDI & high-density interconnect \\
\hline HDL & high-density lipcprotein \\
\hline HOM & higher order mocle \\
\hline IHEP & Institute of High Energ;' Physics \\
\hline IRFEL & infrared free electron laser \\
\hline ISL & IsoSpin Laboratory \\
\hline KRIPES & k-resolved inverse photoemission spectroscopy \\
\hline LANL & Los Alamos National Laboratory \\
\hline LBL & Lawrence Berkeley Laboratory \\
\hline LCAT & lecithin:cholesterol acyltransferase \\
\hline LDL & low-density lipoprotein \\
\hline LDRD & Laboratory Directed Research Development (Program) \\
\hline LNT & liquid nitrogen temperature \\
\hline MBE & molecular beam epitaxy \\
\hline MCD & magnetic circular dichroism \\
\hline MOCVD & metal-organic chemical vapor deposition \\
\hline MQT & macroscopic quantum tunneling \\
\hline MSGC & Microstrip Gas Chamber \\
\hline MSM & metal-semiconductor metal \\
\hline MWPC & multiwire proportional chamber \\
\hline NAPL & nonaqueous phase liquid \\
\hline NMR & nuclear magnetic resonance \\
\hline NSLS & National Synchrotron Light Source \\
\hline
\end{tabular}




$\begin{array}{ll}\text { NSOM } & \text { near-field scanning optical microscope } \\ \text { PCJ } & \text { Pulsed Combustion Jet } \\ \text { PCR } & \text { polymerase chain reaction } \\ \text { PES } & \text { photoelectron spectroscopy } \\ \text { PG\&E } & \text { Pacific Gas \& Electric Company } \\ \text { PIC } & \text { Polymorphism Information Content } \\ \text { POET } & \text { Parallel Project-Oriented Environment and Toolkit } \\ \text { PSG } & \text { protein system graph } \\ \text { PSI } & \text { Primordial Structure Investigation } \\ \text { QPC } & \text { quantum point contact } \\ \text { R\&D } & \text { research and development } \\ \text { RCS } & \text { Rapid Cycling Synchrotron } \\ \text { RNA } & \text { ribonucleic acid } \\ \text { RT } & \text { room temperature } \\ \text { SASE } & \text { self-amplified spontaneous-emission } \\ \text { SCRF } & \text { superconducting radio-frequency } \\ \text { SE } & \text { selenium } \\ \text { SEM } & \text { scanning electron microscopy } \\ \text { SMD } & \text { Silicon Microstrip Disk } \\ \text { SOBP } & \text { spread-out Bragg peak } \\ \text { SONET } & \text { synchronous optical network } \\ \text { SR } & \text { synchrotron radiation } \\ \text { SSRL } & \text { Stanford Synchrotron Radiation Laboratory } \\ \text { STM } & \text { scanning tunneling microscopy } \\ \text { SWORD } & \text { Small Workshop for Optical Resonator Design } \\ \text { SXRFM } & \text { synchrotron x-ray fluorescence microprobe } \\ \text { TEM } & \text { transmission electron microscopy } \\ \text { THG } & \text { third harmonic generation } \\ \text { TPC } & \text { Time Projection Chamber } \\ \text { TPPE } & \text { two-photon photoemission } \\ \text { UC3C } & \text { Utility-Customer 3C } \\ \text { UHV } & \text { ultra-high vacuum } \\ \text { UV } & \text { ultraviolet } \\ \text { VUV } & \text { vacuum ultraviolet } \\ \text { XANES } & \text { x-ray absorption near-edge structure spectroscopy } \\ \text { XAS } & \text { x-ray absorption spectroscopy } \\ \text { XPS } & \text { x-ray photoelectron spectroscopy } \\ \text { XRF } & \text { x-ray fluorescence } \\ \text { XSW } & \text { x-ray standing wave } \\ & \end{array}$



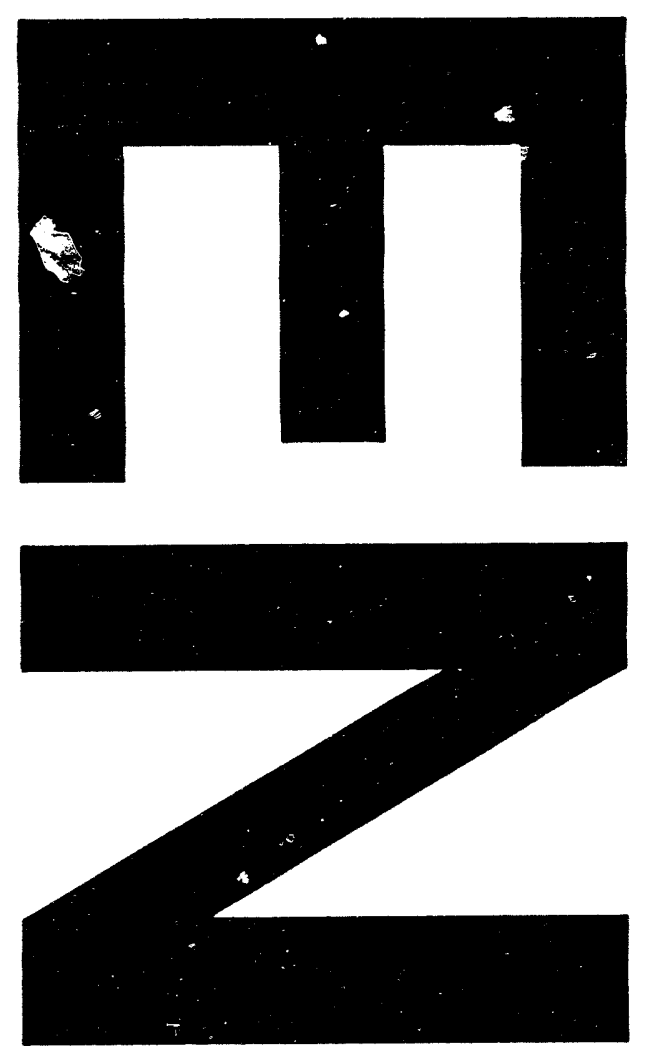

F
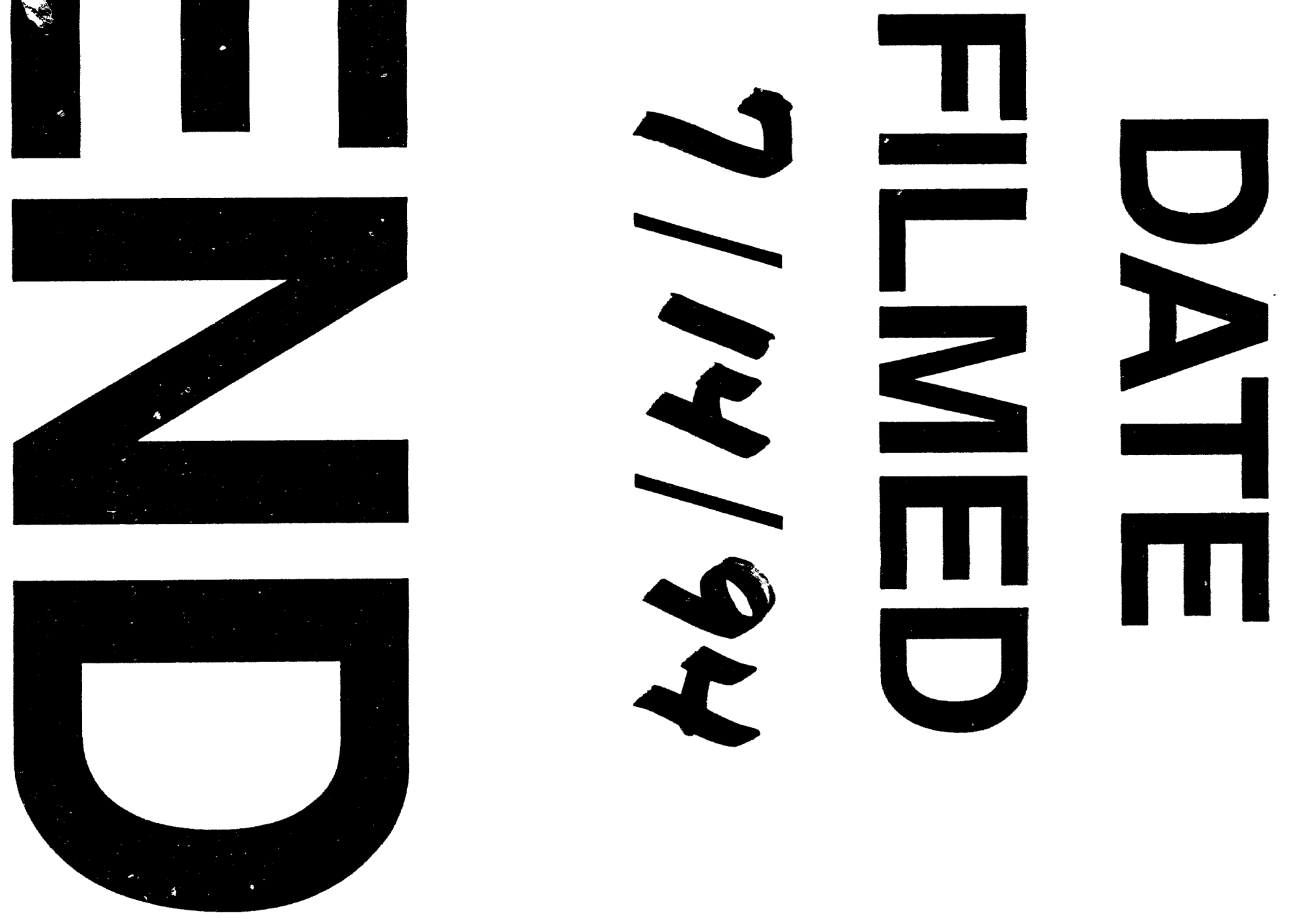

七 II 
